\title{
Degrees of Disordered Eating and Perfectionism in College Students: A Comparison of Sex and Athletic Status
}

\author{
Melinda K. Graham-Hinners \\ West Virginia University
}

Follow this and additional works at: https://researchrepository.wvu.edu/etd

\section{Recommended Citation}

Graham-Hinners, Melinda K., "Degrees of Disordered Eating and Perfectionism in College Students: A Comparison of Sex and Athletic Status" (2013). Graduate Theses, Dissertations, and Problem Reports. 3628.

https://researchrepository.wvu.edu/etd/3628

This Dissertation is protected by copyright and/or related rights. It has been brought to you by the The Research Repository @ WVU with permission from the rights-holder(s). You are free to use this Dissertation in any way that is permitted by the copyright and related rights legislation that applies to your use. For other uses you must obtain permission from the rights-holder(s) directly, unless additional rights are indicated by a Creative Commons license in the record and/ or on the work itself. This Dissertation has been accepted for inclusion in WVU Graduate Theses, Dissertations, and Problem Reports collection by an authorized administrator of The Research Repository @ WVU.

For more information, please contact researchrepository@mail.wvu.edu. 
Degrees of Disordered Eating and Perfectionism in College Students:

A Comparison of Sex and Athletic Status

\section{Melinda K. Graham-Hinners} \\ Dissertation submitted to the \\ College of Education and Human Services \\ at West Virginia University \\ in partial fulfillment of the requirements \\ for the degree of \\ Doctor of Philosophy \\ in \\ Counseling Psychology \\ Jeffrey Daniels, Ph.D., Chair \\ James Bartee, Ph.D. \\ D.J. Hendricks, Ph.D. \\ Ed Jacobs, Ph.D. \\ Jack Watson, Ph.D.
}

Department of Counseling, Rehabilitation Counseling, and Counseling Psychology

Morgantown, West Virginia

2013

Keywords: Disordered Eating, Perfectionism, College Students, Body Image, Eating Disorders, Athletes, Sex 


\section{ABSTRACT \\ Degrees of Disordered Eating and Perfectionism in College Students: A Comparison of Sex and Athletic Status}

\section{Melinda K. Graham-Hinners}

Most research on disordered eating behaviors and body image concerns have focused on females, as females tend to have a higher prevalence of these behaviors than males; however, over the past decade there has been an increase in the number of males presenting with disordered eating behaviors. There has been a debate over whether eating disorders should be viewed as only diagnosable versus non-diagnosable or through a continuum model, with people being categorized as having a diagnosable eating disorder, disordered eating behaviors, or no eating disorder symptoms. Numerous recent studies have supported examining eating disorders from a continuum perspective. An association between perfectionism and disordered eating has been found by several researchers. A great deal of research has been completed on the link between perfectionism and disordered eating among females, however there has been a shortage examining this relationship among males. There has also been a debate over whether perfectionism is a unidimensional or multidimensional construct, with most recent research supporting the multidimensional view of perfectionism. There has also been research completed which suggests that athletes may be at a higher risk for developing disordered eating behaviors than non-athletes, although again most of this research has focused on females and used a discontinuum model of disordered eating and a unidimensional perspective of perfectionism. The present study examined the degrees of disordered eating and perfectionism among college students, comparing sex (males and females) and athletic status (athletes and non-athletes), using a continuum model of disordered eating and a multidimensional approach to perfectionism. This study surveyed 322 college-aged students, 101 female non-athletes, 100 male non-athletes, 53 male athletes, and 68 female athletes. Data analyses revealed that total and maladaptive perfectionism scores significantly varied across the three disordered eating categories, while adaptive perfectionism did not vary across the categories. Results also indicated a statistically significant difference between males' and females' frequencies in the categories of disordered eating (asymptomatic, symptomatic), although the differences between athletes and non-athletes and between male athletes, female athletes, male non-athletes, and female non-athletes were not statistically significant. Finally, data analyses revealed no statistically significant interactions on total, maladaptive, or adaptive perfectionism between or among sex, athletic status, and disordered eating categories; however there were significant main effects for disordered eating category on both total and maladaptive perfectionism. The results of this study lend evidence to the perspectives of perfectionism as a multidimensional construct and the continuum model of disordered eating with college students. Strengths and limitations of this study along with suggestions for future research and clinical applications are discussed. 


\section{Acknowledgments}

I would like to take this opportunity to thank several individuals for the support, knowledge, and guidance they have provided me throughout my doctoral training and the dissertation process. First, I would like to express my sincere thanks and gratitude to Jeffrey Daniels, $\mathrm{PhD}$, for his willingness to serve as my dissertation chair for the last steps of my dissertation. I deeply appreciate his efforts, thoughtfulness, and quick response to emails; without his guidance, I would not have been able to complete this document. I would also like to thank Roy Tunick, EdD, for his guidance in the first part of my dissertation, including the proposal of my dissertation. I would also like to express my sincere thanks to James Bartee, $\mathrm{PhD}$, for his constant guidance, support, encouragement, and belief in me, not only through the dissertation process, but also in my overall doctoral training. I would also like to thank my other committee members, Jack Watson, PhD, Ed Jacobs, PhD, and Deborah Hendricks, EdD, for their helpful suggestions and valuable feedback. I am particular appreciative of Dr. Hendricks' willingness to step in and serve as my statistician committee member at the very last stage of my dissertation. Her enthusiasm to share her expertise in statistical analysis and her availableness in helping to complete the document made the completion of this document possible.

There are several people who made the dissertation process possible given the number of miles between me and my committee. Vicki Railing provided me with constant support, encouragement, and friendship throughout my doctoral training and also played an integral role in ensuring that all steps in the dissertation process were completed. Cathy Jasper graciously agreed to hand-deliver copies of my dissertation to all of my committee members prior to both the proposal and defense meetings and also has provided me with a life-long friendship that I deeply treasure. Finally, I would like to express my sincere thanks to my "West Virginia family," Steve, Andrea, and Brittany Drake. They provided me with housing, meals, constant support and encouragement, and much love throughout my doctoral training and continue to do so today.

I would also like to thank several people who provided consultation, support, and guidance throughout the dissertation process. Several of my students assisted in scoring questionnaires and entering data. My sincere thanks to Tom Grandy, my internship supervisor, who helped with revisions and provided encouragement in the completion of this document. Keith Jones, $\mathrm{PhD}$, provided integral support in my data analysis, for which I am grateful. I would like to thank my undergraduate advisor and colleague, Peggy Fitch, for teaching me that I don't have to be perfect. I am also grateful for my mentor, Ed Willis', constant support, encouragement, and lifelong friendship. Sincere thanks to my friend Tiffany Thomas for her presentation revisions and support. I would also like to thank the administration at William Penn University for supporting the completion of my degree. I have enormous gratitude for my doctoral cohort for their continued support and encouragement and for making my time in the doctoral program so enjoyable.

I would also like to thank my stepson Max for his understanding and flexibility while I was finishing my degree and his constant encouragement even though he is the child and I am the parent. Last, but not least, I am forever indebted to my partner, Jason. His support was unwavering and his patience was immeasurable; I would not have accomplished this without him. 
Table of Contents

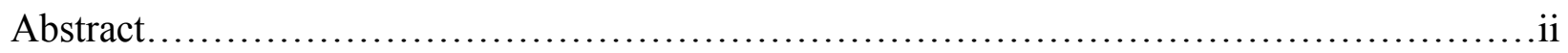

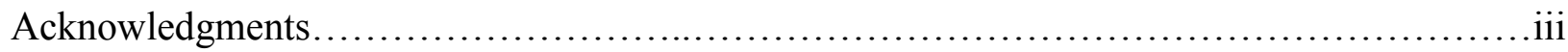

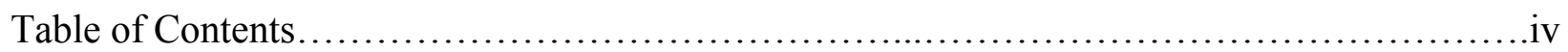

List of Tables and Graphs..........................................................

Chapter 1: Introduction............................................................

Chapter 2: Literature Overview................................................22

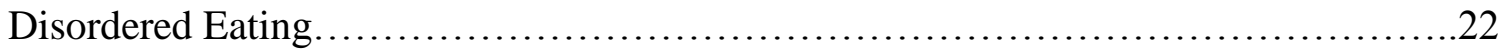

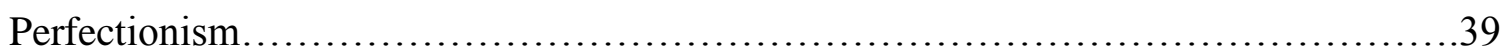

Athletes........................................................................ 61

Chapter 3: Method............................................................... 82

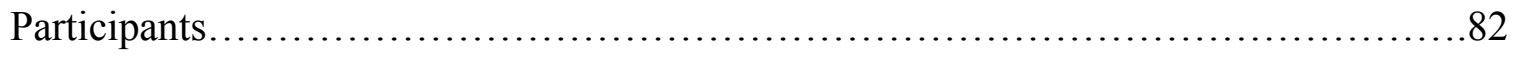

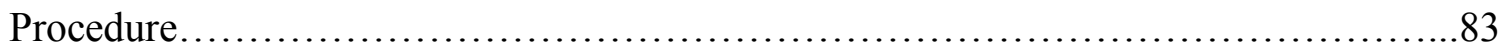

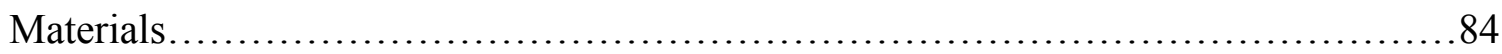

Questionnaire for Eating Disorder Diagnosis (Q-EDD) $\ldots \ldots \ldots \ldots \ldots \ldots \ldots \ldots \ldots . . . \ldots 4$

Frost Multidimensional Perfectionism Scale (Frost MPS).......................90

Data Analysis.............................................................. 93

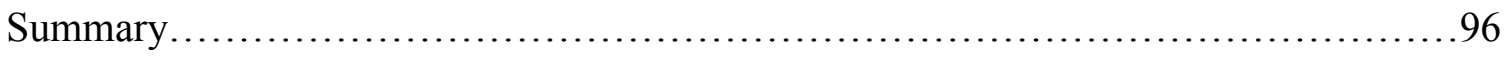

Chapter 4: Results........................................................... 98

Participant Characteristics................................................ 98

Results of Statistical Analyses............................................ 103

Research Question 1a.................................................104 
Research Question 1b............................................ 106

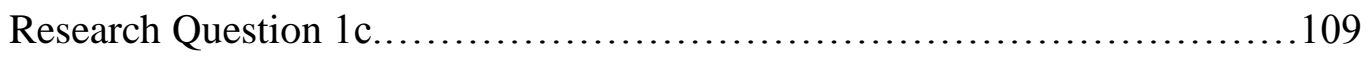

Research Question 2a................................................. 110

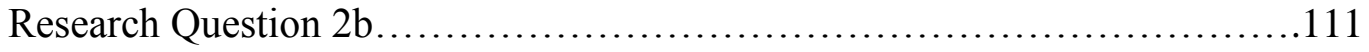

Research Question 2c..............................................113

Research Question 3a..............................................115

Research Question 3b.................................................116

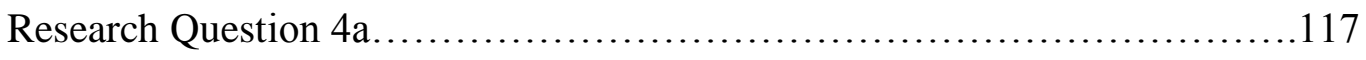

Research Question 4b............................................118

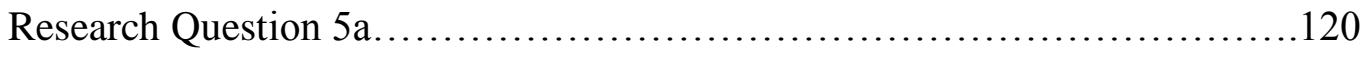

Research Question 5b........................................... 121

Research Question 6a............................................122

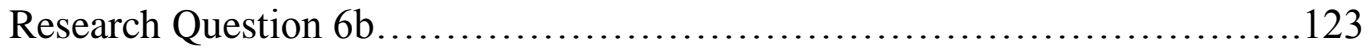

Summary of the Results.................................................. 125

Chapter 5: Discussion........................................................ 127

Findings................................................................. 127

Strengths and Limitations.............................................. 136

Recommendations and Suggestions for Future Research and Clinical Applications.....142

Participants..................................................... 143

Measures................................................................. 145

Clinical Applications................................................146

Summary and Conclusions............................................. 150

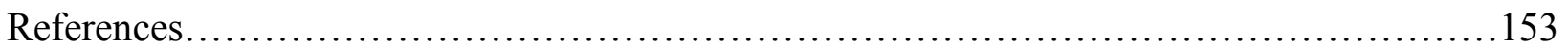


Appendices..................................................................... 161

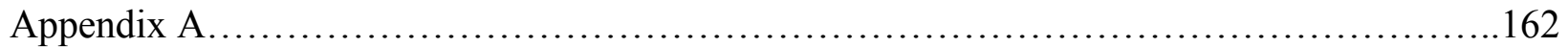

Questionnaire for Eating Disorder Diagnosis (Q-EDD) ...........................163

Frost Multidimensional Perfectionism Scale (Frost MPS)........................167

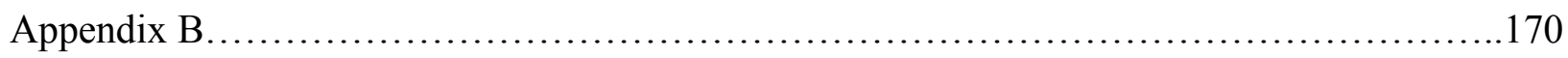

Data Analyses Graphs..................................................... 171 


\section{List of Tables}

$\begin{array}{lll}\text { Table } 1 . & \text { Age } & 99\end{array}$

$\begin{array}{lll}\text { Table 2. Class Status } & 100\end{array}$

$\begin{array}{lll}\text { Table 3. } & \text { Race } & 101\end{array}$

$\begin{array}{lll}\text { Table } 4 . & \text { Non-Athlete Race } & 102\end{array}$

$\begin{array}{lll}\text { Table 5. } & \text { Athlete Race } & 103\end{array}$

Table 6. Descriptive Statistics for Total Perfectionism and Disordered Eating 104

Table 7. Summary of Total Perfectionism and Disordered Eating 105

Table 8. Pairwise Comparison for Total Perfectionism based on Disordered Eating

$\begin{array}{ll}\text { Category } & 105\end{array}$

Table 9. Descriptive Statistics for Maladaptive Perfectionism and Disordered

$\begin{array}{ll}\text { Eating } & 107\end{array}$

Table 10. Summary of Maladaptive Perfectionism and Disordered Eating $\quad 107$

Table 11. Pairwise Comparison for Maladaptive Perfectionism based on Disordered

$\begin{array}{ll}\text { Eating Category } & 108\end{array}$

Table 12. Descriptive Statistics for Adaptive Perfectionism and Disordered Eating 109

Table 13. Summary of Adaptive Perfectionism and Disordered Eating 110

Table 14. Descriptive Data for Chi-Square for Sex and Disordered Eating Category 111

Table 15. Chi-Square Results for Sex and Disordered Eating Category 111

Table 16. Descriptive Data for Chi-Square for Athletic Status and Disordered

$\begin{array}{ll}\text { Eating Category } & 112\end{array}$

Table 17. Chi-Square Results for Athletic Status and Disordered Eating Category 113

Table 18. Descriptive Data for Chi-Square for Sex/Athletic Status in Disordered 
Table 19. Chi-Square Results for Sex/Athletic Status and Disordered Eating

$$
\text { Category }
$$

Table 20. Summary of Total Perfectionism Interactions

Table 21. Summary of Adaptive and Maladaptive Perfectionism Interactions

Table 22. Descriptive Statistics for Total Perfectionism Scores in Males and

Females

Table 23. Summary of Total Perfectionism Main Effect for Sex

Table 24. Descriptive Statistics for Maladaptive and Adaptive Perfectionism

Scores in Males and Females

Table 25. Summary of Maladaptive and Adaptive Perfectionism Main Effect for

Sex

Table 26. Descriptive Statistics for Total Perfectionism Scores in Athletes and

Non-Athletes

Table 27. Summary of Total Perfectionism Main Effect for Athletic Status

Table 28. Descriptive Statistics for Maladaptive and Adaptive Perfectionism

Scores in Athletes and Non-Athletes

Table 29. Summary of Maladaptive and Adaptive Perfectionism Main Effect for Athletic Status

Table 30. Descriptive Statistics for Total Perfectionism Scores in Asymptomatic

DE Category and Symptomatic DE Category

Table 31. Summary of Total Perfectionism Main Effect for Disordered Eating 
Table 32. Descriptive Statistics for Maladaptive and Adaptive Perfectionism

Scores in Asymptomatic and Symptomatic DE Categories

Table 33. Summary of Maladaptive and Adaptive Perfectionism Main Effect for DE Category 
List of Figures

$\begin{array}{lll}\text { Figure 1. } & \text { Total Perfectionism Means } & 106\end{array}$

$\begin{array}{lll}\text { Figure 2. } & \text { Maladaptive Perfectionism Means } & 108\end{array}$

$\begin{array}{ll}\text { Figure 3. } & 110\end{array}$ 


\section{Chapter 1}

\section{Disordered Eating}

Most research on disordered eating behaviors and body image concerns have focused on females, as females tend to have a higher prevalence of these behaviors than males (Cohen \& Petrie, 2005; Field et al., 1999; Grigg, Bowman, \& Redman, 1996; Mintz \& Betz, 1988). Anorexia Nervosa (AN), a diagnosable eating disorder, is characterized by weighing $85 \%$ less than expected for height and age, fear of gaining weight or becoming fat, disturbed perception of body weight or shape, and amenorrhea in females (American Psychiatric Association, 2000). Bulimia Nervosa $(\mathrm{BN})$, another diagnosable eating disorder, is characterized by recurrent episodes of binge eating which includes eating a larger amount of food than normal in a discrete time period and feeling a lack of control over this behavior, recurrent inappropriate compensatory behaviors, binges and compensatory behaviors occur at least twice per week for at least three months, self-evaluation is overly influenced by body weight or shape, and none of these behaviors occur during the presence of AN (American Psychiatric Association, 2000). Binge-Eating Disorder (BED), which currently falls into the Eating Disorder Not Otherwise Specified diagnosis, is characterized by recurrent episodes of binge eating without the compensatory behaviors found in $\mathrm{BN}$ (American Psychiatric Association, 2000). Over 90\% of AN cases occur in females and at least $90 \%$ of $\mathrm{BN}$ cases occur in females (American Psychiatric Association, 2000). BED is slightly more even with the ratio of men to women being 2 to 3 (Robb \& Dadson, 2002). Based on their results, Mintz and Betz concluded that a high percentage of female undergraduates participated in some form of behavior that was related to dieting, with $82 \%$ partaking in one or more behavior on a daily basis and $33 \%$ participating in more serious behaviors such as self-induced vomiting, laxative use, or diet pill use on a monthly basis. They 
only classified 33\% of the females in their study as being normal eaters (Mintz \& Betz, 1988). Grigg et al. also found similar results with high percentages of females participating in binge eating, vomiting to lose weight, and several other unsafe dieting practices such as fasting and the use of diuretics and laxatives.

Even though eating behaviors and body image concerns seem to inflict females more than males, an increasing percentage of eating disorder cases are males (Gary, 2001; Joiner, Katz, \& Heatherton, 2000; O’Dea \& Abraham, 2002). In fact, the first documented case of anorexia nervosa occurred in a 16-year-old male (Morton, 1964 as cited in Mickalide, 1990). As eating disordered behaviors and symptomology is increasing in males, it is imperative that researchers include males in their studies. O’Dea and Abraham examined eating disorders in college males. From a self-report survey, they found that $9 \%$ of the males had disordered eating behaviors, $18 \%$ had weight control problems, and $22 \%$ were binge-eaters; however, none of these males sought treatment due to the stigma attached to males with eating disorders seeking treatment since eating disorders are seen as a female disorder (O’Dea \& Abraham, 2002).

Several researchers have conducted studies comparing males' and females' disordered eating behaviors (Crisp \& Burns, 1990; Edwin \& Andersen, 1990; Furnham, Badmin, \& Sneade, 2002; Hay, Loukas, \& Philpott, 2005; Mickalide, 1990; Presnell, Bearman, \& Madeley, 2007; Varnado-Sullivan, Horton, \& Savoy, 2006; Woodside, Garner, Rockert, \& Garfinkel, 1990). When studying characteristics of those with a diagnosable eating disorder, most researchers have found no difference between males and females, based on various eating disorder measures such as the Eating Attitudes Test (EAT-26) and the Eating Disorder Inventory (EDI) (Crisp \& Burns, 1990; Edwin \& Andersen, 1990; Hay et al., 2005; Woodside et al., 1990). When examining disordered eating behaviors, researchers have concluded that females engage in more disordered 
eating behaviors than males (Furnham et al., 2002; Varnado-Sullivan et al., 2006). However, a few researchers have found that the rates of body dissatisfaction are high in both males and females (Mickalide, 1990; Presnell, et al., 2007; Varnado-Sullivan et al., 2006).

\section{Disordered Eating Continuum}

Moving on from sex differences in disordered eating behaviors, there has been much debate over whether eating disturbances should be studied from a continuum or discontinuum perspective. Researchers have found support for the continuum model of eating disturbances for women (Cohen \& Petrie, 2005; Tylka \& Subich, 1999), however support for the continuum model in males is conflicting (Tylka \& Subich, 2002). Recent research conducted by SundgotBorgen and Torstveit, (2004) found evidence that more people, both males and females, met criteria for subclinical eating disorders than for clinical eating disorders, which would lend support to viewing eating disturbances on a continuum for both males and females. Hausenblas and Carron (1999) encouraged researchers to use the Questionnaire for Eating Disorder Diagnosis (Q-EDD) due to the measure differentiating between those who have no symptoms of an eating disorder (asymptomatic), some symptoms of an eating disorder (symptomatic), and a diagnosable eating disorder (eating disordered).

Mintz and Betz (1988) studied only females and classified participants into different categories based on their eating behaviors and then examined differences between the categories. Those classified as bulimic were found to think about their weight more often and be more fearful of becoming obese (Mintz \& Betz, 1988). Mintz and Betz also found that participants classified as bulimic were more dissatisfied with their body than those classified as bingers, purgers, or subthreshold bulimics, and participants in those three categories were more dissatisfied than those classified as normal. Cohen and Petrie (2005) used an eating behaviors 
measure in addition to the Questionnaire for Eating Disorder Diagnosis (Q-EDD) and found that the eating disorder group was the most disturbed, followed by the symptomatic group, and finally the asymptomatic group having the least disturbances. Overall, Cohen and Petrie concluded that the eating disordered, symptomatic, and asymptomatic groups differed from each other on a majority of variables in a linear fashion, which supports the continuum model of disordered eating.

\section{Disordered Eating and Perfectionism}

Eating problems have long been linked to perfectionism (Ashby, Kottman, \& Schoen, 1998; Bastiani, Rao, Weltzin, \& Kaye, 1995; Bulik et al., 2003; Davis, Claridge, \& Fox, 2000; Halmi et al., 2000; Hewitt, Flett, \& Ediger, 1995; Macedo et al., 2007; McVey, Pepler, Davis, Flett, \& Abdolell, 2002; Parker \& Adkins, 1995; Pliner \& Haddock, 1996; Pratt, Telch, Labouvie, Wilson, \& Agras, 2001; Rohwer \& Massey-Stokes, 2001; Slane, Burt, \& Klump, 2010; Woodside et al., 2004). Vohs, Bardone, Joiner, and Abrahamson (1999) state "the very nature of eating disorders - relentlessly striving toward an impossible standard of thinness - is perfectionistic" (p. 695). A majority of this literature has focused on females, as is the case with research on eating disorders discussed above. Also, there have been many measures of perfectionism used in the various research studies, which had an impact on the findings. For example, some researchers use a unidimensional model of perfectionism, while others use a multidimensional model of perfectionism, which has influenced whether perfectionism is linked to disordered eating or not and also which types of perfectionism are linked to disordered eating behaviors.

\section{Multidimensional Perfectionism}


There is debate over whether to view the construct of perfectionism as a unidimensional or multidimensional construct. Frost, Marten, Lahart, and Rosenblate (1990) stated that past research emphasized "the setting of excessively high personal standards of performance" as the most prominent feature of perfectionism (p. 450). The problem they noted with this thinking is that it does not separate people who are perfectionistic from those who are successful and competent, as it views perfectionism as a unidimensional construct rather than a multidimensional construct (Frost et al., 1990). Many researchers have found perfectionism to be a multidimensional construct (Clavin, Clavin, Gayton, \& Broida, 1996; Frost, Heimberg, Holt, Mattia, \& Neubauer, 1993; Frost et al., 1990; Hewitt \& Flett, 1991 as cited in Parker \& Adkins, 1995). Franco-Paredes, Mancilla-Diaz, Vazquez-Arevalo, Lopez-Aguilar, and Alvarez-Rayon, (2005) stated that by using a multidimensional construct of perfectionism, it helps discriminate between different features of perfectionism.

Hamachek (1978) separated perfectionism into two separate categories: normal perfectionism and neurotic perfectionism. He described normal perfectionists as setting high standards, receiving pleasure from their efforts, and those "who feel free to be less precise as the situation permits" (Hamachek, 1978, p. 27). Normal perfectionists are able to consider both their strengths and weaknesses and set more realistic expectations for themselves (Hamachek, 1978). On the other hand, neurotic perfectionists are seen as 'never good enough' in their own eyes, no matter how successful their efforts (Hamachek, 1978). They typically "demand of themselves a higher level of performance than is usually possible to attain" (Hamachek, 1978, p. 27). Another big difference between normal and neurotic perfectionists is that normal perfectionists tend to be motivated by "a desire for improvement" whereas neurotic perfectionists tend to be motivated by "fear of failure" (Hamachek, 1978, p. 28). 
Similarly, Bardone-Cone et al. (2007) viewed perfectionism as multidimensional and labeled the two types maladaptive and adaptive, which are described in a similar way as Hamachek's neurotic and normal perfectionism, respectively. Using the Frost Multidimensional Perfectionism Scale (Frost MPS), maladaptive perfectionism constitutes the concern over mistakes (CM), parental expectations (PE), parental criticism (PC), and doubts about actions (D) subscales and adaptive perfectionism constitutes the personal standards (PS) and organization (O) subscales (Bardone-Cone et al., 2007). Given the research cited, it seems that a multidimensional view of perfectionism would be able to better discriminate between the positive and negative features associated with perfectionism.

\section{Unidimensional and Multidimensional Research}

When using a unidimensional perspective of perfectionism, Joiner, Heatherton, Rudd, and Schmidt (1997) found that perfectionism was a risk factor for developing Bulimia Nervosa (BN) in females if the female perceived herself to be overweight; however, Rosenvinge, Borgen, and Borresen (1999) did not find a difference in degrees of perfectionism in females classified as having a clinical or subclinical eating disorder compared to those classified as not having an eating disorder. When comparing males and females using a unidimensional perspective of perfectionism, researchers have found similar amounts of perfectionism in males and females

overall (Forbush, Heatherton, \& Keel, 2007; McCabe \& Vincent, 2003). Joiner et al. (1997) and McCabe and Vincent both found perfectionism to have a link to symptoms of BN in males, but not females. Although Forbush et al. found no difference in amounts of perfectionism between males and females overall, they did find that amounts of perfectionism were related to different disordered eating behaviors in each of the sexes. As is evidenced, there are mixed results when using a unidimensional measure of perfectionism. 
When studying females using a multidimensional perspective of perfectionism, there have also been some mixed results, but a majority of the findings lend to maladaptive perfectionism, but not adaptive perfectionism, being associated with disordered eating behaviors or eating disorders (Ashby et al., 1998; Bulik et al., 2003; Hewitt et al., 1995; Pliner \& Haddock, 1996; Pratt et al., 2001). These studies have examined a specific disorder (i.e., AN, BN, or BED) (Bulik et al., 2003; Pliner \& Haddock, 1996; Pratt et al., 2001), a mixture of diagnosable eating disorders (Ashby et al., 1998) and various disordered eating behaviors (Davis et al, 2000.; Hewitt et al., 1995). A handful of studies have found both maladaptive and adaptive perfectionism to be related to disordered eating behaviors or eating disorders in females (Bastiani et al., 1995; Halmi et al., 2000; Hewitt et al., 1995), with all three investigating diagnosable eating disorders. And finally, one study conducted by McVey et al. (2002) found disordered eating behaviors to be related to adaptive perfectionism, but not maladaptive perfectionism in females, which goes against the current study hypothesis.

In addition to general findings about perfectionism being connected to eating disorders and disordered eating behaviors in females, other interesting and relevant pieces of information have been found. Not only is perfectionism prevalent in females who are currently diagnosed with an eating disorder, it has also been found that perfectionism scores tend to be higher for recovered anorexics than for controls (Bardone-Cone et al., 2007; Bastiani et al., 1995), and for those recovered from BN compared to controls (Bardone-Cone et al., 2007). An interesting finding by Halmi et al. (2000) was that the amount of perfectionism increased when the eating disorder was at its worst (i.e. lower body weight, greater preoccupation with eating behaviors, and diminished motivation to change). They concluded that the greater the severity of the eating 
disorder, the higher the amount of perfectionism (Halmi et al., 2000), which lends to viewing eating disorders on a continuum when studying disordered eating behaviors and perfectionism.

Few studies have investigated sex differences in degrees of perfectionism using a multidimensional perspective within a disordered eating or eating disorder population (Macedo et al., 2007; Slane et al., 2010; Woodside et al., 2004). Woodside et al. found that males with an eating disorder had lower amounts of perfectionism than females with an eating disorder, but did not analyze the difference between adaptive and maladaptive perfectionism. Slane et al. examined one subscale of the Frost Multidimensional Perfectionism Scale (Frost MPS), concern over mistakes (CM), that is included in the calculation of maladaptive perfectionism and found it to be related to disordered eating behaviors in both males and females. Macedo et al. found total perfectionism to be related to disordered eating for their sample of males and females, and when broken down, maladaptive perfectionism to be related to disordered eating in males and both adaptive and maladaptive perfectionism to be related to disordered eating in females.

There has also been limited research on the degree of perfectionism differing across the continuum of eating disorders (Tylka \& Subich, 1999; Tylka \& Subich, 2002). Tylka and Subich (1999) found that based on the perfectionism scale of the Eating Disorder Inventory (EDI) (unidimensional), females placed into the three categories on the Questionnaire for Eating Disorder Diagnosis (Q-EDD) (asymptomatic, symptomatic, and eating disordered) did not differ on degrees of perfectionism. Tylka and Subich (2002) used males as participants, and also based on the perfectionism scale of the EDI found that males placed into the three categories of disordered eating on the Q-EDD did not differ on degrees of perfectionism. These findings are contradictory to the current study hypothesis; however, a unidimensional perspective of 
disordered eating was used in these studies rather than a multidimensional perspective as described above.

\section{Athletes}

As reviewed above, many studies have found a link between perfectionism and disordered eating behaviors, using female participants, unidimensional measures of perfectionism, and a discontinuum model of eating disorders. However, there are some special populations that may be at higher risk for both disordered eating behaviors and perfectionism that have not received much research attention, one of these populations being athletes. Many athletes are under pressure to perform at a high standard and in turn be successful in their athletic endeavors. Within some sports, these standards of performance are tied to certain body types, such as being lean or having a low body fat percentage. In order to obtain these body types, many athletes will engage in unhealthy eating behaviors, such as bingeing, purging, restrictive eating, and laxative abuse among others (Byrne \& McLean, 2001).

Several researchers have found that athletes have more disordered eating behaviors compared to non-athletes (Byrne \& McLean, 2001; Hausenblas \& Carron, 1999; Johnson, Powers, \& Dick, 1999; Petrie \& Rogers, 2001; Pritchard, Milligan, Elgin, Rush, \& Shea, 2007; Smolak, Nurnen, \& Ruble, 2000; Sundgot-Borgen \& Torstveit, 2004; Szymanski \& Chrisler, 1990-1991). Smolak et al. found female athletes engaged in disordered eating behaviors more than female non-athletes in their meta-analysis, Byrne \& McLean in their literature review found mixed results on whether female athletes had higher frequency of disordered eating than female non-athletes, and Fulkerson, Keel, Leon, and Dorr (1999) found no difference in degree of disordered eating behaviors between female athletes and non-athletes. When studying male athletes compared to male non-athletes, most studies have found that male athletes engaged in 
disordered eating behaviors more than male non-athletes (Hausenblas \& Carron, 1999; Pritchard et al., 2007; Sundgot-Borgent \& Torstveit, 2004), with the exception of Pritchard et al. finding male non-athletes to have more body dissatisfaction than male athletes and Fulkerson et al. finding no difference between male athletes and non-athletes on degree of disordered eating behaviors. Pritchard et al., using the suggested cutoff score of 20 to determine an eating disorder on the Eating Attitudes Test (EAT), found that only $13 \%$ of female non-athletes qualified as having an eating disorder, whereas $36 \%$ of female athletes met the criteria, and $1 \%$ of male nonathletes met the cutoff for an eating disorder compared to $9 \%$ of male athletes, demonstrating that symptoms of eating disorders are higher among both female and male athletes when compared to same-sex control groups.

Some studies have suggested that using the continuum model of eating disorders, as described above, with athletes is supported with diagnosable eating disorder participants having the most symptoms, athletes having fewer symptoms, and a control group having the least symptoms (Byrne \& McLean, 2001). Support for the continuum model has also been found by Petrie and Rogers (2001) and Sundgot-Borgen and Torstveit (2004) who both concluded that subclinical eating disorders were more prevalent among athletes than non-athletes.

Several researchers have found that female athletes engaged in more disordered eating behaviors than male athletes (Byrne \& McLean, 2001; Johnson et al., 1999; Pritchard et al., 2007; Sundgot-Borgen \& Torstveit, 2004). The same researchers found the difference in disordered eating behaviors between female athletes and non-athletes to be greater than the difference between male athletes and non-athletes; however, Hausenblas and Carron (1999) found that the difference in disordered eating behaviors between male athletes and non-athletes was greater than the difference between female athletes and non-athletes. 
Being successful at athletics and having disordered eating behaviors have both been linked to perfectionism (Byrne \& McLean, 2001). Using only females, Szymanski and Chrisler (1990-1991) found that athletes had higher degrees of perfectionism than non-athletes. Fulkerson et al. (1999) used both males and females and overall found a couple of differences in disordered eating behaviors between those with high compared to low perfectionism scores. The problem with these findings is that perfectionism was measured using a unidimensional model and disordered eating behaviors were measured using a discontinuum model.

As the pressure on athletes to be successful and perform at high standards continues to exist, it is important to investigate how athletes are making this success happen, especially in relation to disordered eating behaviors. Along with this, it is important to determine if athletes are more likely to display symptoms of disordered eating than non-athletes, and if this differs by sex (male or female). It is also important to examine whether the amounts of perfectionism (total, adaptive, and maladaptive) are higher in athletes than non-athletes or differs by sex, as this may give some insight into the desire to achieve these high standards and be successful. In summary, it is imperative that research be conducted on female and male athletes and non-athletes using both a multidimensional model of perfectionism and a continuum model of disordered eating to assess the degree of perfectionism and disordered eating behaviors and to see if these differ among athletes compared to non-athletes or in females compared to males.

\section{Statement of the Problem}

Traditionally, research on disordered eating behaviors has focused on females, with a few recent studies attending to disordered eating in males. Within the research on disordered eating, there have been very few studies that utilize a continuum model of disordered eating. These few 
studies supported utilization of this model with females; however, the support for using the continuum model with males was conflicting.

The link between disordered eating behavior and perfectionism has been well researched. Almost all of the research linking these two variables has been conducted using female participants. With a disordered eating population, studies have produced inconsistent results on the issue of differences in amount of perfectionism between males and females. Recent research has tended towards increasing support of the multidimensional model of perfectionism within disordered eating populations. The explanation for this trend towards using the multidimensional model of perfectionism involved a more complete explanation of how perfectionism functions for the individual research participants.

There have been inconsistencies in the results of studies examining the relationship between athletic status and disordered eating behaviors. Some studies have found no relationship between these two variables, while other studies have found disordered eating behaviors to be more prevalent in athletes than non-athletes. These contradictory findings may be due in part to the different age groups (adolescents, young adults) and athletic levels (high school, collegiate, elite) in these studies, as disordered eating and perfectionism may be more common in some age groups or athletic levels than others. Disordered eating behaviors were more prevalent in female athletes compared to male athletes in a majority of the studies. With the high degree of pressure placed on both male and female athletes to be successful, it is imperative to investigate if athletes are engaging in disordered eating behaviors more than non-atheltes in order to be successful and if this is different based on their amount of different types of perfectionism. Determining if athletes score higher on the dimensions of perfectionism (total, adaptive, and maladaptive) compared to non-athletes and have more symptoms of disordered eating behaviors compared to 
non-athletes may help to explain their drive to be successful and encourage athletes and their supporters to find safer methods of achieving this success. Also, determining if this differs between males and females can help determine who all is in need of learning safer and healthy methods with regards to both disordered eating behaviors and perfectionism.

Based upon the current research findings, it appears that females tend to have a higher amount of perfectionism and disordered eating behaviors than males. A less conclusive finding suggests that athletes may have higher amounts of perfectionism and disordered eating than nonathletes, with female athletes exhibiting higher amounts of perfectionism and disordered eating behaviors than male athletes. These findings, however, have been based upon various measures of disordered eating behaviors and perfectionism, usually a discontinuum model of disordered eating and a unidimensional perspective of perfectionism. Recent research has supported measuring disordered eating behaviors on a continuum versus concrete specific diagnoses. Given these findings, it is imperative to examine disordered eating behaviors in collegiate athletes and non-athletes using the continuum model. With the recent research support for a multidimensional model of perfectionism (adaptive and maladaptive), it also seems important to revisit degrees of perfectionism in collegiate athletes and non-athletes using a strictly multidimensional model of perfectionism. In summary, it is useful to examine the amounts of perfectionism and disordered eating comparing sex (male and female) and athletic status (athlete and non-athlete), using a continuum model of disordered eating behaviors and a multidimensional model of perfectionism.

\section{Summary}

The proposed study is designed to examine the amounts of perfectionism and disordered eating behaviors among college students, comparing sex (male and female) and athletic status (athlete and non-athlete). The current proposed study will utilize a continuum model of 
disordered eating and a multidimensional view of perfectionism. By using a continuum model of disordered eating, the study will be able to differentiate between those with a diagnosable eating disorder (eating disordered), those with symptoms of an eating disorder (symptomatic), and those who have no symptoms of an eating disorder (asymptomatic) and to see if this differs based on sex and athletic status. By using a multidimensional perspective of perfectionism, the study will be able to differentiate total perfectionism, maladaptive perfectionism, and adaptive perfectionism to see if the amounts of each vary between sexes and athletic statuses, or if there is one specific type of perfectionism that is more common among one sex or athletic status compared to the other. The use of these two perspectives also allows the study to determine if there is a difference in amounts of the different types of perfectionism based on the category of disordered eating behavior. Finally, the study will either lend evidence to or undermine the perspectives of perfectionism as a multidimensional construct and the continuum model of eating disorders with collegiate athletes.

\section{Purpose of the Current Study}

The present study is designed to determine the amounts of disordered eating (asymptomatic, symptomatic, and eating disordered) and perfectionism (total, adaptive, and maladaptive) in college students. Specifically, the study is designed to establish if scores on the three types of perfectionism vary across the disordered eating categories; if males and females, athletes and non-athletes, and male athletes, female athletes, male non-athletes, and female nonathletes differ in their frequencies in the disordered eating categories (asymptomatic, symptomatic); and if there are significant differences on perfectionism scores (total, maladaptive, adaptive) between males and females, athletes and non-athletes, and the asymptomatic and symptomatic categories of disordered eating. 
Research Question 1a: Do scores on total perfectionism vary across disordered eating categories (asymptomatic, symptomatic, eating disordered)? (Measured by the Frost Multidimensional Perfectionism Scale (Frost MPS) and the Questionnaire for Eating Disorder Diagnosis (Q-EDD))

Hypothesis 1a: Scores on total perfectionism do vary across disordered eating categories (asymptomatic, symptomatic, eating disordered), with the asymptomatic category having the lowest mean total perfectionism scores, followed by the symptomatic category, and finally the eating disordered category having the highest mean total perfectionism scores.

Research Question 1b: Do scores on maladaptive perfectionism vary across disordered eating categories (asymptomatic, symptomatic, eating disordered)? (Measured by the Frost MPS and the Q-EDD)

Hypothesis $1 \mathrm{~b}$ : Scores on maladaptive perfectionism do vary across disordered eating categories (asymptomatic, symptomatic, eating disordered), with the asymptomatic category having the lowest mean maladaptive perfectionism scores, followed by the symptomatic category, and finally the eating disordered category having the highest mean maladaptive perfectionism scores.

Research Question 1c: Do scores on adaptive perfectionism vary across disordered eating categories (asymptomatic, symptomatic, eating disordered)? (Measured by the Frost MPS and the Q-EDD)

Hypothesis 1c: Scores on adaptive perfectionism do not vary across disordered eating categories (asymptomatic, symptomatic, eating disordered), with all three disordered eating categories (asymptomatic, symptomatic, eating disordered) having approximately the same mean adaptive perfectionism scores. 
Research Question 2a: Does a difference exist in the frequencies between males' and females' in the disordered eating categories (asymptomatic, symptomatic)? (Measured by the Q-EDD)

Hypothesis 2a: A difference does exist in the frequencies between males' and females' in the disordered eating categories (asymptomatic, symptomatic), with more males in the asymptomatic category, and more females in the symptomatic category.

Research Question 2b: Does a difference exist in the frequencies between non-athletes' and athletes' in the disordered eating categories (asymptomatic, symptomatic)? (Measured by the QEDD)

Hypothesis 2b: A difference does exist in the frequencies between non-athletes' and athletes' in the disordered eating categories (asymptomatic, symptomatic), with more nonathletes in the asymptomatic category and more athletes in the symptomatic category. Research Question 2c: Does a difference exist in the frequencies between male athletes', female athletes', male non-athletes', and female non-athletes' in the disordered eating categories (asymptomatic, symptomatic)? (Measured by the Q-EDD)

Hypothesis 2c: A difference does exist in the frequencies between male athletes' female athletes', male non-athletes', and female non-athletes' in the disordered eating categories (asymptomatic, symptomatic). More specifically, there will be more male athletes than male non-athletes in the symptomatic category, more female athletes than female non-athletes in the symptomatic category, more female athletes than male athletes in the symptomatic category, and more female non-athletes than male non-athletes in the symptomatic category.

Research Question 3a: Are there any significant interactions on total perfectionism scores between or among the independent variables of sex, athletic status, and disordered eating category? (Measured by the Frost MPS and the Q-EDD) 
Hypothesis 3a: There are significant interactions on total perfectionism scores between or among the independent variables of sex, athletic status, and disordered eating category. Research Question 3b: Are there any significant interactions on maladaptive or adaptive perfectionism scores between or among the independent variables of sex, athletic status, and disordered eating category? (Measured by the Frost MPS and the Q-EDD)

Hypothesis $3 b$ : There are significant interactions on maladaptive perfectionism scores between or among the independent variables of sex, athletic status, and disordered eating category; however, there are no significant interactions on adaptive perfectionism scores between or among the independent variables of sex, athletic status, and disordered eating category. Research Question 4a: Is there a significant difference on total perfectionism scores between males and females? (Measured by the Frost MPS)

Hypothesis 4a: There is a significant difference on total perfectionism scores between males and females, with females having higher total perfectionism scores than males. Research Question 4b: Is there a significant difference on maladaptive or adaptive perfectionism scores between males and females? (Measured by the Frost MPS)

Hypothesis $4 b$ : There is a significant difference on maladaptive perfectionism scores between males and females, with females having higher maladaptive perfectionism scores than males; however, there is no significant difference on adaptive perfectionism scores between males and females.

Research Question 5a: Is there a significant difference on total perfectionism scores between athletes and non-athletes? (Measured by the Frost MPS) 
Hypothesis 5a: There is a significant difference on total perfectionism scores between athletes and non-athletes, with athletes having higher total perfectionism scores than nonathletes.

Research Question 5b: Is there a significant difference on maladaptive or adaptive perfectionism scores between athletes and non-athletes? (Measured by the Frost MPS)

Hypothesis $5 b$ : There is a significant difference on maladaptive perfectionism scores between athletes and non-athletes, with athletes having higher maladaptive perfectionism scores than non-athletes; however, there is no significant difference on adaptive perfectionism scores between athletes and non-athletes.

Research Question 6a: Is there a significant difference on total perfectionism scores between the asymptomatic and symptomatic categories of disordered eating? (Measured by the Frost MPS and the Q-EDD)

Hypothesis 6a: There is a significant difference on total perfectionism scores between the asymptomatic and symptomatic categories of disordered eating, with the symptomatic category having higher total perfectionism scores than the asymptomatic category.

Research Question 6b: Is there a significant difference on maladaptive or adaptive perfectionism scores between the asymptomatic and symptomatic categories of disordered eating? (Measured by the Frost MPS and the Q-EDD)

Hypothesis $6 b$ : There is a significant difference on maladaptive perfectionism scores between the asymptomatic and symptomatic categories of disordered eating, with the symptomatic category having higher maladaptive perfectionism scores than the asymptomatic category; however, there is no significant difference on adaptive perfectionism scores between the asymptomatic and symptomatic categories of disordered eating. 


\section{Importance of the Study}

By assessing the amount of disordered eating behaviors and different types of perfectionism in college students (males and females and athletes and non-athletes), useful information will be provided in order to ensure that the college students are using safe and healthy methods to achieve success on or off the athletic field. Many colleges and universities implement programming with their students, specifically athletes, in order to prevent unhealthy behaviors such as disordered eating. The results of this study could lend support to creating more of these programs addressing health ways to achieve success that do not include disordered eating behaviors. By investigating whether different types of perfectionism are more common among one sex (male or female) or athletic status (athlete or non-athlete), and especially those that may partake in disordered eating behaviors, we can start to determine which type(s) of perfectionism are common among this population and therefore, start developing programming to address this issue as well as disordered eating behaviors.

Studies have attempted to study these two variables within male and female collegiate athletes and non-athletes; however, none of them have used both a continuum model of disordered eating and a multidimensional perspective of perfectionism. Recent research supports the use of these two models when studying these variables, and therefore it is important that studies be completed using measures that assess a continuum of disordered eating and different types of perfectionism. By only studying those who have a diagnosable eating disorder compared to those that do not have a diagnosable eating disorder, a large population of people who engage in disordered eating behaviors is either being left out or included in the non-diagnosable group, which may not be different enough than the diagnosable group. For example, if someone met some criteria for one of the diagnosable eating disorders, but not all criteria, they would be put in 
the non-diagnosable group; however, they may have some very similar characteristics to the diagnosable group due to the symptoms of disordered eating they display. When researchers viewed perfectionism from a unidimensional model, they were examining both the positive and negative aspects of perfectionism as all one variable rather than separating out the positive aspects of perfectionism (adaptive) and the negative aspects of perfectionism (maladaptive). In order to get a more detailed and clear picture of these two variables in college students, it is necessary to investigate the continuum of disordered eating behaviors and the different types of perfectionism.

\section{Scope of the Study and Definitions}

There are various age groups and levels of athletes that could be examined; however, studying all of them is not within the scope of this proposed study. The study will focus on male and female collegiate athletes and non-athletes at a Midwestern Division I school. Participants will vary in ranking in college (e.g., freshman, sophomore, junior, and senior) in order to get the most representative sample possible. The sample will be gathered through a simple convenience method and cluster convenience method. The proposed study is also somewhat limited in the scope of disordered eating behaviors it covers. It will not be examining specific behaviors such as dieting or fasting, but will rather place individuals into one of three categories of disordered eating behaviors (asymptomatic, symptomatic, or eating disordered) that will be used to describe the degree of disordered eating. The proposed study is relatively broad in scope with regards to perfectionism, as amounts of total, adaptive, and maladaptive perfectionism will be examined, which agrees with current research supporting the multidimensional nature of perfectionism.

For the purposes of this study, athletes will be defined as individuals who are currently members of a NCAA athletic team on the specified university campus. Non-athletes will be 
defined as individuals who are not currently members of a NCA athletic team on the specified university campus. Perfectionism is defined by a person's scores on the Frost Multidimensional Perfectionism Scale (Frost MPS). More specifically, total perfectionism will be defined as the sum of the concern over mistakes (CM), personal standards (PS), parental expectations (PE), parental criticism (PC), and doubts about actions (D) subscales of the Frost MPS. Adaptive perfectionism will be defined as the sum of the PS and organization $(\mathrm{O})$ subscales of the Frost MPS. Maladaptive perfectionism will be defined as the sum of the CM, PE, PC, and D subscales of the Frost MPS. Disordered eating will be defined by the placement into one of the following three categories from the Questionnaire for Eating Disorder Diagnosis (Q-EDD): asymptomatic, symptomatic, or eating disordered, using the scoring model provided by the authors of the QEDD. 


\section{Chapter 2 - Literature Review}

\section{Disordered Eating}

As stated in the introduction, most of the research conducted on disordered eating has focused on females. A majority of this research has found similar results, namely, that females engage in many different disordered eating behaviors such as dieting, fasting, self-induced vomiting, and laxative use among others, in addition to having a distorted body image. Given the overwhelming support from research of the prevalence of disordered eating behaviors and distorted body image among females, a small selection of studies will be reviewed here as an example of the current research knowledge. Some of the current research regarding males and disordered eating behaviors will be reviewed next. Because of the recent increase in males with body image issues, researchers have started conducting studies on males and disordered eating behaviors, although there is not an abundance of research in this area. A majority of this section will be devoted to literature reviewing the comparison between males and females concerning disordered eating behaviors and body image concerns. The most attention is given to this section due to the current study comparing males and females. Finally, articles will be reviewed that provide justification for viewing eating disorders on a continuum rather than a discontinuum.

Grigg, Bowman, and Redman (1996) investigated the frequency of several disordered eating behaviors, including dieting and body image distortion; using 869 girls aged 14-17. Each participant completed a questionnaire that measured weight control methods, degrees of body image satisfaction, and disordered eating behaviors, and also had their height and weight taken in order to calculate body mass index (BMI) (Grigg et al., 1996). Using the BMIs of participants, $3.8 \%$ were classified as obese, $12 \%$ as overweight, $54.6 \%$ as acceptable, $29.7 \%$ as underweight, and $5.9 \%$ as very underweight (Grigg et al., 1996). Grigg et al. found that $33 \%$ of their 
participants engaged in at least one disordered eating behavior, including binge eating, vomiting, and dieting when underweight. Of the participants, Grigg et al. found that $47 \%$ were currently attempting to lose weight and $64 \%$ had attempted to lose weight in the past year. A disturbing finding was that $19 \%$ of the underweight students were currently attempting to lose weight (Grigg et al., 1996). With regards to dieting, Grigg et al. found that $57 \%$ of the participants practiced unhealthy dieting (cutting foods, skipping meals, crash or fad diets, fasting, or using dietary supplements), while $36 \%$ practiced extreme dieting (crash diets, fasting, diet pills, diuretics, laxatives, and cigarettes). Overall, $12 \%$ of the participants were labeled as having a distorted body image (perceived weight category vs. actual BMI deviating by 2 or more categories) (Grigg et al., 1996). Another interesting finding by Grigg et al. was that approximately two-thirds of the participants perceived themselves as being overweight, when in reality only $16 \%$ were actually overweight. Given the findings by Grigg et al., it is imperative that disordered eating behaviors be studied in order to gain knowledge and possibly develop programs to prevent these behaviors. Grigg et al. chose to study high school females in their research, which provides good information; however, is somewhat limited in the age group and sex of the participants. They also relied on self-report surveys to collect data, which can always cause some issues with honesty in reporting, but even more so with this population given their need to impress friends and "fit in." The questionnaire that was used in the Grigg et al. study was designed by the researchers for this specific research project; although this may give them valuable information, it may not be a reliable and/or valid measure given it was designed specifically for this study and no information was provided on the reliability and validity of the measure (Grigg et al., 1996). 
Mintz and Betz (1988) examined similar behaviors as Grigg et al. (1996) using 682 college aged females. In addition to investigating disordered eating behaviors such as weight management and body image, Mintz and Betz also examined self-esteem and other sociocultural influences on disordered eating behaviors. All participants completed two questionnaires regarding disordered eating behaviors, a self-esteem measure, a measure about attractiveness related to sociocultural norms, and a demographic measure which included questions about body image including perceived weight category (Mintz \& Betz, 1988). Thirty-one participants were removed from the data analysis due to either being classified as anorexic ( 8 participants) or obese (31 participants) (Mintz \& Betz, 1988). Mintz and Betz found that a high percentage of undergraduate women engaged in some form of dieting behavior, with approximately $28 \%$ engaging in dieting behaviors more than once daily and 54\% participating in dieting behaviors on a daily basis. Mintz and Betz found that participation in more extreme behaviors, such as laxative use and self-induced vomiting, occurred for $2 \%$ of the participants daily, while $8 \%$ partook in these activities on a weekly basis, and $19 \%$ on a monthly basis. Thirty-eight percent of participants admitted to binge-eating, $75 \%$ admitted to eating low calorie food, $30 \%$ admitted to fasting, and 20\% admitted to using appetite control pills (Mintz \& Betz, 1988). These statistics are alarming; as it appears that college aged women have very high rates of disordered eating behaviors. The results found by Mintz and Betz, similar to the results from Grigg et al., demonstrate the importance of studying disordered eating behaviors. As with the Grigg et al. study, however, Mintz and Betz only used female participants and used self-report measures. Mintz and Betz also reported that their participants were primarily first and second year college students, which limits the generalizability to other populations (non-college) and age groups. 
Researchers have recently begun to take interest in males and disordered eating behaviors, as the prevalence of these behaviors is on the rise among males (Robb \& Dadson, 2002). Robb and Dadson investigated different eating disorders that impact males, differences in males' perception of body image and dieting, and specific groups at high-risk for disordered eating behaviors among males. An interesting finding was that most males are dissatisfied with their body in one of two ways: they either want to be thinner or they want to have greater muscle mass (Robb \& Dadson, 2002). Similar to females, it has been found that many males diet due to a self-perception of being overweight when in reality they are not overweight (Robb \& Dadson, 2002). Herbold and Frates (as cited in Robb \& Dadson) found that approximately $25 \%$ of males had tried to lose weight in the past week and approximately $1.5 \%$ had reported using selfinduced vomiting as a means of weight control. Robb and Dadson reported that males make up anywhere from 2-8\% of AN cases, 3-10\% of BN cases, approximately one-third of BED cases, and almost all of the muscle dysmorphobia cases. Robb and Dadson had a broad scope in their review, as they investigated several eating disorders and also several disordered eating behaviors. They also reviewed studies completed on a broad range of ages (elementary school aged children to adult) and from many regions of the world (i.e. Canada, South Africa, Australia). Their review demonstrated some of the variations found within the research on males and disordered eating behaviors. Given that Robb and Dadson did not complete their own research study, it is unknown if the measures used in the studies they reviewed viewed eating disorders from a discontinuum perspective, with scales reflecting disordered eating behaviors (i.e. drive for thinness, dieting) or a continuum measure that would distinguish between those with diagnosable eating disorders, those with symptoms of an eating disorder, and those without any symptoms of an eating disorder. 
O’Dea and Abraham (2002) examined disordered eating behaviors using 93 college aged males. Participants completed one questionnaire on eating and exercise behaviors and then answered three questions concerning disordered eating, weight control, and binge eating (O'Dea and Abraham, 2002). O'Dea and Abraham found that one-fifth of the participants reported significant concern about their weight and shape and engaged in restrictive eating behaviors on a regular basis. Based on self-report, O’Dea and Abraham found that $9 \%$ of participants had disordered eating issues, $18 \%$ had weight control problems, and 22\% had a binge-eating problem. Overall, O’Dea and Abraham reported that 20\% of their participants were participating in behaviors or had attitudes that were indicative of disordered eating or an eating disorder. The researchers also noted that none of their participants had ever sought treatment for these attitudes or behaviors, and hypothesized that this may be due to a societal stigma that these issues only impact women (O'Dea and Abraham, 2002). The results from this study indicate that disordered eating behaviors are as important of an issue among males as they are among females given that $20 \%$ of the participants were participating in behaviors or had attitudes that were indicative of an eating disorder, which provides justification for studying these behaviors among males in the future. O'Dea and Abraham's results are valuable in understanding the prevalence of disordered eating behaviors with males. One possible limitation is that they used a measure that supports a discontinuum model of eating disorders, with subscales that may touch on some of the disordered eating behaviors, rather than a measure supporting a continuum model of disordered eating behaviors.

A study completed by Kearney-Cooke and Steichen-Asch (1990) investigated body image issues and risk factors for eating disorders using 112 college aged males and 16 male clinical patients. The 112 college aged participants first completed a questionnaire on body shape 
in order to determine their group placement: at-risk, determined by scoring in the range typical of eating-disordered patients, and normal, determined by scoring lower than eating-disordered patients (Kearney-Cooke \& Steichen-Asch, 1990). This resulted in 28 males in the at-risk category and 84 in the normal category (Kearney-Cooke \& Steichen-Asch, 1990). Every participant then completed an eating attitudes test, a personality inventory, and a demographic questionnaire related to body image history (Kearney-Cooke \& Steichen-Asch, 1990). KearneyCooke and Steichen-Asch found that males in all three categories (clinical, at-risk, and normal) reported being ashamed of their "'stomach, gut, belly"” followed by "'upper and lower extremities"” (p. 57). Kearney-Cooke and Steichen-Asch found that the normal and at-risk groups felt the ideal male body was muscular and strong, whereas the clinical group felt it was thin and lean. As expected, there was a significant difference in degrees of body satisfaction, with the normal group being most satisfied, followed by the at-risk group, and finally the clinical group being the least satisfied (Kearney-Cooke \& Steichen-Asch, 1990). This study provides useful information, especially in discriminating between a clinical population, at-risk population, and normal population and differences in their body image perception. A strength of this study is bringing in a clinical population for comparison with the at-risk and normal groups; however the sample size of the clinical group is rather small. Also, they used a cutoff score on a body shape questionnaire that was "typical" of eating-disordered patients' scores to classify at-risk individuals versus normal individuals. Although this may be useful, there is no guarantee that it is accurate, as the measure is not designed to categorize people as at-risk versus normal.

As a result of the increase in prevalence of disordered eating behaviors in males, researchers have begun to compare males and females to find similarities and differences in prevalence rates, disordered eating behaviors, age of onset, and other relevant factors. Woodside 
et al. (1990) conducted a study comparing the clinical presentation and psychometric profiles of 20 males diagnosed with eating disorders to a sample of 20 females diagnosed with similar eating disorders. They interviewed all of the male participants and administered two eating disorder assessments and one body image satisfaction assessment (Woodside et al., 1990). Woodside et al. (1990) found that males had significantly lower scores on the dieting subscale of the Eating Attitudes Test (EAT) compared to females; however, male and female scores did not differ on the bulimia and oral control subscales. Overall on the EAT, Woodside et al. (1990) found that females scored higher; however, a large percentage of both males (75\%) and females (90\%) scored above the cutoff score of 20. The Eating Disorder Inventory (EDI) was also administered by Woodside et al. (1990), which is comprised of 8 subscales. Woodside et al. (1990) found that the only significant difference was on the drive for thinness subscale, with all other subscales being similar for males and females. They also found no significant differences between males and females on the body image satisfaction questionnaire (Woodside et al., 1990). Woodside et al. (1990) concluded that males and females diagnosed with eating disorders are more similar than dissimilar. The results from the Woodside et al. (1990) study are extremely informative in showing that the clinical presentation of males and females with eating disorders are very similar. One limitation of this study is the small sample size (20 males and 20 females). Another limitation is that all of the participants had a clinical diagnosis of an eating disorder (AN or $\mathrm{BN})$, which does not give any information on differences between males and females who may have disordered eating behaviors, but not meet diagnostic criteria for an eating disorder. As in previous studies reviewed, the measures used by Woodside et al. (1990) were all discontinuum model measures of eating disorders, with subscales that touch on disordered eating behaviors 
compared to a continuum model measure that separates people by disordered eating categories based off of their behaviors (i.e. eating disordered, symptomatic, and asymptomatic).

Edwin and Andersen (1990) performed a study examining males with eating disorders over a 10-year period and compared them to a reference group of females with eating disorders. The participants were all given a multitude of assessments, including a clinical interview, two eating disorder assessments, and a health assessment (Edwin \& Andersen, 1990). Edwin and Andersen assigned participants to one of two groups: primary eating disorders, which included $\mathrm{AN}$ and $\mathrm{BN}$, and secondary eating disorders, which included people referred for an eating disorder, but diagnosed with another disorder such as depressive or anxiety disorders. Edwin and Andersen had 54 participants in the primary group and 20 in the secondary group, and used 84 females with either AN or BN as the reference group. Some differences found between males and females included males being older than what is typically reported for female eating disordered patients, and the average weight of males prior to the onset of their disorder was higher than females compared to the ideal body weight for each (Edwin \& Andersen, 1990). Edwin and Andersen also found that males tend to want a more muscular or toned physique while females want to weigh less. There was no difference found by Edwin and Andersen on frequency of bingeing or Eating Attitudes Test (EAT) scores between males and females. On the Eating Disorder Inventory (EDI), Edwin and Andersen found males and females only differed on the body dissatisfaction scale, with females being more dissatisfied with their bodies than males, which is different than the findings by Woodside et al. (1990), who found the only difference to be on the drive for thinness subscale of the EDI. Again, as with Woodside et al. (1990), Edwin and Andersen only compared males and females with diagnosable eating disorders, not giving evidence for similarities or differences between males and females with disordered eating 
behaviors that would not necessarily qualify as a diagnosable eating disorder. Also similar to the Woodside et al. (1990) study, discontinuum model measures of eating disorders were used in this study. Even though some of the participants were classified as having an eating disorder based off of their scores on these measures, which is what they are designed for, they were compared to a group with other psychiatric disorders (depressive disorders, anxiety disorders), who may or may not show signs of disordered eating behaviors.

Crisp and Burns (1990) compared clinical features in males and females diagnosed with AN, using two studies. One study compared 36 males and 102 females and the other study had 29 males and 23 females (Crisp \& Burns, 1990). Crisp and Burns found that the mean age of presentation was similar for males and females, contradicting the findings of Edwin and Andersen (1990). Crisp and Burns found that males were more overweight than females at the onset of their diagnosis, which agrees with the findings by Edwin and Andersen. They also found that both males and females were concerned about their weight and being fat (Crisp \& Burns, 1990). Crisp and Burns found a significant difference in reported appetites during childhood, with $9 \%$ of females reporting a poor appetite and $53 \%$ a big appetite compared to $19 \%$ of males with a poor appetite and $22 \%$ with a big appetite. In one study, a follow-up was completed and more males were below $90 \%$ of target weight than females; however, there had been two female deaths and no male deaths (Crisp \& Burns, 1990). Crisp and Burns concluded that the clinical features present during AN are similar for males and females. As stated above, this study has some similar findings as earlier reviewed studies and some contradictory findings, although both Crisp and Burns and Edwin and Andersen concluded that males and females are more similar than different. One major difference, however, is that Crisp and Burns only used individuals diagnosed with $\mathrm{AN}$, rather than also using individuals diagnosed with $\mathrm{BN}$. One limitation of the 
study is the drastic difference in numbers of males and females used. And again, only people with diagnosable eating disorders, specifically AN, were studied, not giving information about possible similarities and differences between males and females with disordered eating behaviors compared to a diagnosable eating disorder.

Hay et al. (2005) conducted a study that investigated the characteristics of males with eating disorders compared to females with eating disorders. The researchers randomly selected 500 males and 500 females that attended two university clinics and mailed them an eating disorder questionnaire (Hay et al., 2005). Hay et al. then conducted interviews with 50 male participants who met specific criteria, including:

four or more episodes of overeating associated with a loss of control, and/or any episodes of vomiting, or laxative or diuretic use for weight or shape control, and/or scored four or more on the three questions of the EDE-Q that assessed attitudes to weight, shape and food, and/or reported an exercise frequency of greater than three times a week and only if the exercise was done for shape or weight reasons. (p. 2)

During the interview, participants were administered a demographic questionnaire, an eating disorder questionnaire, a neuropsychiatric questionnaire, a symptoms questionnaire, and two questionnaires regarding exercise behaviors (Hay et al., 2005). Data for female comparison came from an earlier study conducted by the researchers (Hay et al., 2005). In the current study, Hay et al. found $1.2 \%$ of the sample had a diagnosable eating disorder, whereas $5.2 \%$ of participants from the earlier female study had a diagnosable eating disorder. The researchers then narrowed their sample to only include those who met criteria for disordered eating, which included 22 males and 36 females from the earlier study, in order to compare disordered eating behaviors between males and females (Hay et al., 2005). Hay et al. found that males and females were 
similar in their Eating Disorders Examination-Questionnaire (EDE-Q) scores, BMI, exercise levels, and laxative use. Females were found to use self-induced vomiting as a compensatory behavior more than males and also have more weight concerns than males (Hay et al., 2005). Hay et al. also found that females tend to seek help for their disordered eating behaviors more than males, which agrees with the findings by O'Dea and Abraham (2002) reviewed above. The findings by Hay et al. support the notion that males and females diagnosed with an eating disorder do not differ significantly when it comes to most disordered eating behaviors, in accordance with the findings by Woodside et al. (1990). This again provides useful information in the comparison of males and females. Once more, a limitation is the small sample size used for the comparison. Another limitation is that the males and females were not studied at the same time, which could cause some issues in the comparison. They also only studied individuals who have been diagnosed with an eating disorder and these individuals may differ from those who suffer from disordered eating behaviors that do not meet criteria for a diagnosable eating disorder.

Varnado-Sullivan et al. (2006) examined differences between males' and females' body image disturbance, disordered eating behaviors, and exercise using 233 female and 200 male college aged participants. Participants completed a demographic questionnaire, which included self-reported weight and height, and questions concerning dieting behaviors and use of supplements, and also completed several questionnaires regarding disordered eating behaviors, body image satisfaction, and exercise behaviors (Varnado-Sullivan et al., 2006). VarnadoSullivan et al. found dieting behaviors between males and females to differ slightly, with $38 \%$ of females currently dieting compared to $20 \%$ of males. There was a significant difference in the use of supplements to enhance muscles, with 60 males and 8 females reporting the use of these 
supplements (Varnado-Sullivan et al., 2006). Varnado-Sullivan et al. also found that males exercise more frequently than do females, with females' primary reason for exercising being losing weight, while males felt better about themselves when engaged in regular exercise activity. Varnado-Sullivan et al. found that males had a more positive body satisfaction, although a high percentage of both males $(62 \%)$ and females $(79 \%)$ wanted to change their body size. As expected, Varnado-Sullivan et al. found that females mainly wanted to decrease their body size; however, males were split relatively equally between wanting to increase their body size (30\%) and wanting to decrease their body size (32\%). Varnado-Sullivan et al. broke the males down into three weight categories: low-normal weight, normal weight, and higher weight, and found that the higher weight group had the highest body image concerns and bulimic-type behaviors. Varnado-Sullivan et al. concluded that females had more disordered eating symptoms and higher body image dissatisfaction than males, but that males also engaged in these behaviors. This study gives useful information about non-eating disorder diagnosed individuals and their perceptions of body image and disordered eating behaviors, concluding that both males and females have body image disturbances and participate in disordered eating behaviors, with females having a higher rate of these behaviors and disturbances. Even though they used participants that did not have a diagnosable eating disorder, the measures that were administered are all from the discontinuum model of eating disorders, mainly used to diagnose eating disorders. The information gathered about disordered eating behaviors was based off of subscales within these questionnaires, which include only a few questions for each behavior. Their inclusion of exercise is an interesting variable, although it would be interesting to see the difference between those who exercise on a regular basis and individuals that are part of an athletic team on a college 
campus, in order to determine if the amounts of disordered eating varies between these two populations.

A study was carried out by Furnham et al. (2002) that investigated the differences between males' and females' body image satisfaction, attitudes towards eating, self-esteem, and exercise behaviors. Furnham et al. studied 111 boys and 124 girls from a high school setting and administered the Eating Attitudes Test (EAT), a self-esteem assessment, a reasons for exercising questionnaire, and a contour drawing scale to assess body image satisfaction. Furnham et al. found some significant differences between boys and girls, including higher weight dissatisfaction, higher rates of abnormal eating attitudes and behaviors, and a larger distinction between actual and ideal body shape among girls than boys. It was also found that girls tended to exercise in order to lose weight, whereas boys tended to exercise for fitness reasons (Furnham et al., 2002), which is in accordance with the findings by Varnado-Sullivan et al. (2006). In relation to exercising, girls who exercised to be attractive had higher disordered eating behaviors, whereas for boys both positive (i.e. fitness) and negative (i.e. attractiveness) reasons for exercising were related to disordered eating behaviors (Furnham et al., 2002). Furnham et al. found that a majority of both boys $(79.9 \%)$ and girls $(81.7 \%)$ had a discrepancy between their perceived body image and ideal body image. In accordance with the results from VarnadoSullivan et al. (2006), Furnham et al. found that a majority of the girls desired a thinner figure, whereas the boys were split relatively evenly between wanting a larger figure $(36.1 \%)$ or a thinner figure $(42.8 \%)$. This study concluded that there was more body image dissatisfaction among girls than boys and also more disordered eating behaviors and attitudes among girls than boys (Furnham et al., 2002). One limitation to this study is that it used a high school aged population, which may lead to the finding of higher rates of body image dissatisfaction and 
disordered eating behaviors and attitudes due to other factors such as peer pressure that may not be as prevalent among other populations. As with all of the previously reviewed studies, they also used a discontinuum measure of eating disorders, rather than one that views disordered eating behaviors on a continuum.

Many of studies conducted on eating disorders have used measures that support a discontinuum model of eating disorders, as is evidenced by the reviews above. These discontinuum measures are designed to diagnose people as either having a diagnosable eating disorder or not having a diagnosable eating disorder. The problem arises when someone has disordered eating behaviors, but does not meet criteria for a diagnosable eating disorder. For example, a person may engage in crash dieting or fasting, but not meet the diagnostic criteria for AN because they are not below $85 \%$ of their ideal weight (based on DSM-IV-TR criteria). In order to study this population that is not diagnosable, yet still has eating issues, many researchers have supported the idea of a continuum model of eating disorders, where people are classified as either having a diagnosable eating disorder, having symptoms of an eating disorder, or being symptom free (Cohen \& Petrie, 2005; Mintz \& Betz, 1988; Tylka \& Subich, 1999; Tylka \& Subich, 2002).

In their study reviewed earlier, Mintz and Betz (1988) investigated disordered eating behaviors by categorizing 643 participants into six groups: normals (32.8\%), chronic dieters (11.4\%), bulimics $(3.1 \%)$, bingers $(15.6 \%)$, purgers $(10.3 \%)$, and subthreshold bulimics $(26.9 \%)$ using the Weight Management, Eating, and Exercise Habits Questionnaire (WMQ), which is designed to categorize participants into the six categories. Mintz and Betz found that the bulimic group was significantly different from all other groups on several variables including body image satisfaction, self-esteem, societal beliefs about thinness and attractiveness, concern about weight, 
and fear of becoming fat. Specifically, when examining body image satisfaction, bulimics were the most dissatisfied, followed by the bingers, purgers, and subthreshold bulimics, and finally the normals that were the most satisfied with their body image (Mintz \& Betz, 1988). On the selfesteem variable, bulimics had lower self-esteem than bingers and subthreshold bulimics, who in turn had lower self-esteem than the normals (Mintz \& Betz, 1988). When examining societal beliefs about thinness and attractiveness, fear of becoming fat, and concerns about weight, bulimics differed significantly from all of the other groups, endorsing societal beliefs about thinness and attractiveness more, being more fearful of becoming fat, and having more weight concerns (Mintz \& Betz, 1988). Overall, Mintz and Betz found that there are some differences between individuals who meet diagnostic criteria for an eating disorder $(\mathrm{BN})$ and those who display disordered eating behaviors. Disturbingly, there were only $33 \%$ of individuals from this study that were classified as normal eaters. This study provides valuable information about the differences between individuals categorized as eating disordered, individuals who display symptoms of an eating disorder, and individuals who are considered normal eaters. This study only focused on college aged women in their first and second year, so may not be generalizable to other populations such as males or other age groups. There are also some disordered eating behaviors, such as fasting and excessive exercise, that were not examined in this study. Cohen and Petrie (2005) examined behaviors associated with disordered eating among 334 undergraduate females using the Questionnaire for Eating Disorder Diagnosis (Q-EDD), which is designed to measure symptoms of eating disorders and place individuals into one of three categories: eating disordered, symptomatic, and asymptomatic. The researchers also administered a demographic questionnaire, which included self-reported weight and height, two other assessments designed to assess eating behaviors, two cognitive assessments, a 
questionnaire about mood and esteem, three scales regarding body attitude, and a social desirability assessment (Cohen \& Petrie, 2005). Based on Q-EDD results, 51.5\% of participants were classified as asymptomatic, $38.9 \%$ as symptomatic, and $9.6 \%$ as eating-disordered (Cohen $\&$ Petrie, 2005). Cohen and Petrie found that those in the eating-disordered group had more bulimic symptoms, more concern about dieting, and greater weight fluctuation than did the symptomatic group, who also displayed higher amounts of these variables than the asymptomatic group. Analysis ran on the cognitive variables found significant differences between the three groups in a linear fashion on most of the variables (Cohen \& Petrie, 2005). Cohen and Petrie also found a linear difference in preoccupation with body shape and low satisfaction with facial features, with the eating-disordered group having the most preoccupation and lowest satisfaction, followed by the symptomatic group, and finally the asymptomatic group. Overall, Cohen and Petrie found that there are significant differences between these three groups, with most of these differences occurring in a stepwise fashion (disordered-eating, followed by symptomatic, followed by asymptomatic). The results of this study lend support for the use of a continuum model when studying eating-disordered behaviors among females, as it evidences differences between those diagnosed with an eating disorder, those who have symptoms of an eating disorder, and those who are symptom free. Again, however, only females were used in this study, so it is unclear if the same results would be found for males.

Tylka and Subich (1999) investigated the use of a measure supporting the continuum model of disordered eating, the Questionnaire for Eating Disorder Diagnosis (Q-EDD) compared to measures supporting the discontinuum model of disordered eating including the Dieting Beliefs Scale (DBS) and the Eating Disorder Inventory (EDI). Tylka and Subich (1999) used 135 high school and college aged females as their participants. All participants completed the Q- 
EDD, EDI, the DBS, and an assessment for social desirability (Tylka \& Subich, 1999). Tylka and Subich (1999) found that participants' scores on eight of the nine EDI subscales varied based on their placement into the three categories of the Q-EDD (eating-disordered, symptomatic, asymptomatic), with the only subscale not varying being perfectionism. Tylka and Subich (1999) also found that participants' scores on the DBS varied according to their placement in the three Q-EDD categories, with all three groups having significantly different scores from each other. These results support the idea that disordered eating behaviors in females should be viewed from a continuum model rather than a discontinuum model, as all three groups differ from each other on typical disordered eating behaviors (Tylka \& Subich, 1999). The major limitations to this study were the small number of participants and that all participants were female.

Tylka and Subich (2002) investigated the use of a continuum model of disordered eating behaviors with males using 166 males from either a high school $(\mathrm{N}=26)$ or college $(\mathrm{N}=140)$. Each participant was administered the Questionnaire for Eating Disorder Diagnosis (Q-EDD), six subscales from the Eating Disorder Inventory (EDI), and the Effectiveness and Safety of Weight Control Techniques questionnaire developed by the researchers (Tylka \& Subich, 2002). Tylka and Subich (2002) found that three of the six EDI subscales administered (ineffectiveness, interoceptive awareness, and maturity fears) varied based on participant placement in the three categories of the Q-EDD, while the other three (interpersonal distrust, body dissatisfaction, and perfectionism) did not vary according to the placements. When investigating safety and effectiveness of weight control techniques, Tylka and Subich (2002) found that long-term effectiveness, short-term safety, and long-term safety varied according to placement into the three categories. The long-term safety was significantly different among all three groups; however, for long-term effectiveness and short-term safety the asymptomatic group differed 
from both the symptomatic and eating-disordered groups, but the symptomatic and eatingdisordered groups did not differ from each other (Tylka \& Subich, 2002). Tylka and Subich (2002) concluded that the results are conflicting as to whether eating disturbances should be viewed on a continuum model in males. Several variables did not differ for males along the continuum, such as body dissatisfaction and interpersonal distrust, which were found to differ for females in an earlier study conducted by Tylka and Subich (1999). Interestingly, perfectionism did not differ for either males or females based on placement in the three categories (Tylka \& Subich, 1999; Tylka \& Subich, 2002). The findings from this study provide contradictory evidence with regards to the discontinuum versus continuum model of eating disorders in males. Further research is needed using the continuum model with males in order to provide evidence one way or the other.

\section{Perfectionism}

Several researchers have found a link between perfectionism and disordered eating behaviors (Ashby et al., 1998; Bastiani et al., 1995; Bulik et al., 2003; Davis et al., 2000; Forbush et al., 2007; Halmi et al., 2000; Hewitt et al., 1995; Joiner et al., 1997; Joiner et al., 2000; Macedo et al., 2007; McCabe \& Vincent, 2003; McVey et al., 2002; Pliner \& Haddock, 1996; Pratt et al., 2001; Rohwer \& Massey-Stokes, 2001; Slane et al., 2010; Woodside et al., 2004). A majority of the research in this area has focused on females, as is common in disordered eating literature. First, there will be a review of the literature focusing on disordered eating behaviors and/or eating disorders and perfectionism using a unidimensional perspective in females. This will be followed by studies comparing males and females with disordered eating or eating disorders using a unidimensional perspective of perfectionism. Most researchers have agreed that perfectionism is best studied from a multidimensional perspective rather than a 
unidimensional perspective, especially when researching disordered eating behaviors and/or eating disorders (Ashby et al., 1998; Bastiani et al., 1995; Bulik et al., 2003; Davis et al., 2000; Halmi et al., 2000; Hewitt et al., 1995; McVey et al., 2002; Pliner \& Haddock, 1996; Pratt et al., 2001; Woodside et al., 2004). Using a multidimensional perspective allows researchers to "distinguish perfectionistic people from those who are highly competent and successful" (Franco-Paredes et al., 2005, p. 62). Given this stance, articles with information on both unidimensional and multidimensional perspectives will be reviewed, followed by articles examining the relationship between disordered eating behaviors and/or eating disorders and perfectionism using a multidimensional approach, starting with those focusing on females only and then those comparing males and females.

Joiner et al. (1997) studied perfectionism and perceived weight status in relation to symptoms of BN using 435 undergraduate females. Each participant completed a demographic questionnaire, including height and weight to calculate BMI, the perfectionism and bulimia subscales of the Eating Disorder Inventory (EDI), questions based on DSM criteria of BN, and categorized themselves as overweight or not overweight in order to determine their perceived weight status (Joiner et al., 1997). Overall, the researchers found that perfectionism was weakly related to $\mathrm{BN}$ symptoms in a positive direction and that perceived weight status had a strong relationship to BN symptoms (Joiner et al., 1997). Joiner et al. also found an interaction between perfectionism and perceived weight status, which was a predictor of BN symptoms. With regards to this interaction, Joiner et al. concluded that females who categorized themselves as overweight and who scored high on perfectionism had the highest frequency of BN symptoms. The researchers then compared females who categorized themselves as overweight versus those who categorized themselves as not overweight and found that perfectionism scores were a significant 
predictor of BN symptoms for those who categorized themselves as overweight, but not for those who categorized themselves as not overweight (Joiner et al., 1997). Joiner et al. also investigated perceived weight status compared to real weight status among females that scored high on perfectionism and found that females who perceived themselves to be overweight, even if they really were not overweight, were at high risk for BN symptoms; whereas those females who did not perceive themselves as overweight, but really were overweight, were not at risk for experiencing BN symptoms. Joiner et al. also conducted a second study using the same measures as described above. The second study was a 10-year follow-up study with a different group of participants, with the average age of the participant approximately 10 years older than in the first study (Joiner et al., 1997). The results of the first study were replicated in the second study, leading Joiner et al. to conclude that "perfectionism appeared to serve as a risk factor for bulimic symptoms for women who perceived themselves as overweight, but not for those who did not" (p. 151). The results of the Joiner et al. study provide important information on the impact of perceived weight status on BN symptoms and the continuation of this impact 10-years follow-up; however, there are several limitations to the studies. Joiner et al. used a unidimensional perspective of perfectionism, which does not allow for distinguishing between adaptive and maladaptive perfectionism. The study also used only females who were currently enrolled in college or were college-educated, which may not be generalizable to other populations such as males, other age groups, or non-college educated individuals.

Rosenvinge et al. (1999) examined the relationship between various eating disorders and psychological variables, including perfectionism, in 15-year-old students. Each participant completed the drive for thinness, bulimia, and body satisfaction subscales of the Eating Disorder Inventory (EDI) and the perfectionism and general dissatisfaction subscales from the Setting 
Conditions for Anorexia and Bulimia Scale (SCANS) (Rosenvinge et al., 1999). In addition, those suspected of having a diagnosable eating disorder based on EDI scores were administered a clinical interview in order to diagnose their respective disorder (Rosenvinge et al., 1999). Rosenvinge et al. found that 19 female participants had a clinical or subclinical eating disorder and grouped these participants together as the eating disorder group when running statistics. These participants were compared to a control group of 68 participants (Rosenvinge et al., 1999). Rosenvinge et al. found that the eating disorder group had significantly higher scores on the general dissatisfaction scale of the SCANS, but did not differ from the control group on the perfectionism scale of the SCANS. The finding of no relationship between perfectionism and eating disorders by Rosenvinge et al. is in contrast to many researchers who have found this link as described in the introduction to this section. Although this provides some contradictory evidence on the relationship between perfectionism and eating disorders, the fact that the participants were 15-year old high school students may have influenced this result. The researchers also stated that they recruited both males and females at the beginning of the study, but only females met the criteria for a clinical or subclinical diagnosis and the researchers do not state whether only females were used in the control group also, or if it was a combination of males and females in the control group. Another limitation is that those who were classified as either clinical or subclinical were grouped together; therefore, it is unclear whether these two groups would have differed on any of the variables.

McCabe \& Vincent (2003) conducted a study that examined binge eating, bulimic symptoms, and weight loss behaviors, and several psychological variables including perfectionism in $7^{\text {th }}$ and $8^{\text {th }}$ grade boys and girls. The participants, 306 girls and 297 boys, completed a self-report questionnaire packet including the Bulimia Test-Revised (BULIT-R), the 
perfectionism and ineffectiveness subscales of the Eating Disorder Inventory (EDI), a selfesteem scale, a scale measuring depression and anxiety symptoms, a scale looking at pubertal development, in addition to obtaining height and weight measurements to calculate BMI (McCabe \& Vincent, 2003). Based on BMI calculations, 5.6\% of the girls and $4.1 \%$ of the boys were classified as underweight (McCabe \& Vincent, 2003). Small percentages (.3-2.3\%) of both boys and girls were found to participate in the following extreme weight loss behaviors: purging, use of diet pills, use of laxatives, and use of diuretics (McCabe \& Vincent, 2003). McCabe and Vincent found that the girls scored significantly higher on frequency of extreme weight loss behaviors than boys, although no differences were found between boys and girls on binge eating and bulimic symptoms. Boys and girls also had similar BMIs and similar scores on the perfectionism scale of the EDI (McCabe \& Vincent, 2003). When examining predictors of disordered eating behaviors, McCabe and Vincent found that perfectionism was a significant predictor of binge eating for boys, but not for girls. Interestingly, perfectionism was found to be a predictor variable in binge eating for males, but not females; however, the participants in this study were $7^{\text {th }}$ and $8^{\text {th }}$ graders, so this may not be generalizable to the college-aged population. The measure of perfectionism used in this study was also unidimensional, therefore, it is unclear whether both adaptive and maladaptive perfectionism are predictors of binge eating or just one of them.

Joiner et al. (2000) also used both males and females when studying different features, including perfectionism, which differentiate the two sexes with regards to BN symptoms. There study used 14 males and 97 females who completed questionnaires at two different times, 10 years apart (Joiner et al., 2000). Each participant completed questionnaires with questions about their height, weight, eating behaviors, concerns about dieting, body weight and shape, and 
symptoms of eating disorders (Joiner et al., 2000). Participants also completed the bulimia, drive for thinness, perfectionism, and interpersonal distrust subscales of the Eating Disorder Inventory (EDI) (Joiner et al., 2000). Joiner et al. (2000) used a MANCOVA with sex as the independent variable and drive for thinness, perfectionism, and interpersonal distrust as dependent variables and found that females with chronic BN symptoms had significantly higher scores on drive for thinness, whereas males with chronic BN symptoms had significantly higher scores on perfectionism and interpersonal distrust. This was in accordance with Joiner et al.'s (2000) predictions and also with past research cited in their study. Joiner et al. (2000) determined their own cut-off score on the EDI bulimia subscale, so that roughly $15 \%$ of the females and five to ten times more females than males were included in the chronic BN symptom group, which may be a limitation due to the differences in severity among this group. Also, the number of males in this study was relatively small, which may have impacted the results.

Forbush et al. (2007) studied the relationship between perfectionism and various disordered eating behaviors among 1732 female and 750 male undergraduates over a course of 20 years. Eight hundred females and 400 males were randomly selected at three different points, 10 years apart, with participation rates of $72 \%$ for females and $63 \%$ for males (Forbush et al., 2007). BMIs were calculated for each participant in addition to each one completing the perfectionism scale of the Eating Disorder Inventory (EDI) and self-report surveys that asked questions regarding history of fasting, binge-eating, self-induced vomiting, diuretic use, or laxative use in order to control weight (Forbush et al., 2007). Forbush et al. found no difference in the amount of perfectionism between males and females overall. Forbush et al. also found that males reported much lower lifetime prevalence rates of disordered eating behaviors than females. In females, histories of fasting, binge-eating, self-induced vomiting, diuretic use, and laxative 
use were all found to be related to higher amounts of perfectionism; whereas in males, only history of fasting was related to higher amounts of perfectionism (Forbush et al., 2007). More specifically, for females both fasting and purging had a stronger relationship with perfectionism than binge-eating, and fasting and purging were not significantly different in their relationship with perfectionism (Forbush et al., 2007). For males, fasting had a stronger relationship with perfectionism than purging, however fasting and binge-eating were not significantly different in their relationship with perfectionism, nor were purging and binge-eating (Forbush et al., 2007). Overall, Forbush et al. concluded that even though there was no significant difference in overall amounts of perfectionism in males and females, the relationship between perfectionism and selfinduced vomiting and purging was stronger for females, while the relationship between perfectionism and fasting and binge-eating was relatively similar for males and females. Forbush's findings reveal useful information when it comes to sex differences in perfectionism and various disordered eating behaviors. Once again, however, a unidimensional approach to perfectionism was used, therefore we do not know if the sex differences in perfectionism associated with various disordered eating behaviors would still exist if a multidimensional perspective of perfectionism were used, and if so, which types of perfectionism would be associated with which behaviors among males and/or females.

As stated in the introduction to this section, most researchers in the area of perfectionism and disordered eating believe perfectionism should be viewed from a multidimensional perspective. Franco-Paredes et al. (2005) believe that a unidimensional view does not separate people who achieve success from those who are perfectionistic. Franco-Paredes et al. also believe that using a multidimensional perspective of perfectionism gives "a detailed description of perfectionistic behaviour, making it possible to distinguish two aspects present in this kind of 
behaviour" (p. 62). These two aspects have been labeled several things including adaptive or normal and maladaptive or neurotic. When developing their Multidimensional Perfectionism Scale, Frost et al. (1990) believed that psychological problems, including disordered eating behaviors, were more related to maladaptive perfectionism, or the critical evaluations of performance, than with adaptive perfectionism, or the setting of high standards.

Bardone-Cone et al. (2007) completed a meta-analysis of 55 different studies that examined the relationship between perfectionism and eating disorders. When addressing the question of whether amounts of perfectionism are higher in people diagnosed with eating disorders compared to a healthy control group, Bardone-Cone et al. found that most studies using a unidimensional perspective of perfectionism concluded that amounts of perfectionism were higher in those with AN than in those without AN. Studies that used a multidimensional perspective found the amounts of both maladaptive and adaptive perfectionism to be higher in those diagnosed with AN than healthy controls (Bardone-Cone et al., 2007). When investigating $\mathrm{BN}$ and $\mathrm{BED}$, studies using a unidimensional perspective found perfectionism amounts to be higher for those with BN compared to healthy controls, but not higher for those with BED than healthy controls (Bardone-Cone et al., 2007). When reviewing studies that used a multidimensional model of perfectionism Bardone-Cone et al. found mixed results with one studying finding amounts of both maladaptive and adaptive perfectionism to be higher in those with $\mathrm{BN}$ than in the control group and another study finding the amounts of adaptive perfectionism to be higher for those with $\mathrm{BN}$ or BED compared to healthy controls, but no significant difference in amounts of maladaptive perfectionism. Overall, Bardone-Cone concluded that research generally shows a relationship between both adaptive and maladaptive perfectionism and eating disorders, although the relationship is stronger for $\mathrm{AN}$ than for $\mathrm{BN}$ or 
BED. Bardone-Cone et al. also compared those who were considered recovered from an eating disorder to those that were currently diagnosed with an eating disorder and found that amounts of perfectionism were similar for recovered $\mathrm{AN}$ and $\mathrm{BN}$ participants and those that were currently diagnosed, which helps support a continuum model of disordered eating as many times individuals recovered from an eating disorder still display symptoms of disordered eating. They concluded that "recovery from $\mathrm{AN}$ and $\mathrm{BN}$ is not accompanied by reductions in either maladaptive or achievement striving perfectionism..." (Bardone-Cone et al., 2007, p. 391). Given this last finding, it is important to examine disordered eating behaviors on a continuum when studying perfectionism, as many individuals who are recovered from a diagnosable eating disorder will still suffer from disordered eating behaviors, and more than likely have higher amounts of perfectionism than those who have never had an eating disorder and do not have any disordered eating behaviors. The conflicting results found by Bardone-Cone et al. in their metaanalysis provides justification for continued studies looking at disordered eating behaviors and perfectionism, as based on their results, it is unclear if amounts of perfectionism vary based on degree of disordered eating.

Pliner and Haddock (1996) completed a study that examined 100 females diagnosed with $\mathrm{AN}$ and their amounts of perfectionism based on being classified as weight concerned and controls. In order to determine which group the participants were in, Pliner and Haddock administered the Eating Attitudes Test (EAT) to the participants and placed those with high EAT scores in the weight concerned group and those with lower EAT scores in the control group. Pliner and Haddock informed participants that they would be coming up with "uses" for specific objects. Each participant was assigned to either the high goal, low goal, or self-defined goal group (Pliner \& Haddock, 1996). Participants in all groups were told to try their best, with those 
in the high goal group being told to come up with at least 12 uses on each trial, and those in the low goal group being told to come up with at least 5 uses on each trial (Pliner \& Haddock, 1996). Pliner and Haddock asked those in the high and low goal groups to repeat how many uses the experimenter wanted them to achieve and then also set a personal goal, while those in the selfdefined goal group were only asked to set a personal goal. Participants were then given either successful feedback (i.e., doing well on the task and meeting the goal) or failure feedback (i.e., not doing well on the task and failing to reach the goal) (Pliner \& Haddock, 1996). The participants each completed 10 trials, which were broken into two blocks (the first five trials and the second five trials) (Pliner \& Haddock, 1996). Pliner and Haddock found that for those in the high goal group, those with higher EAT scores set higher goals than those with lower EAT scores on the second block of trials, confirming their prediction and agreeing with the inference that people with AN "conform to the unrealistically high performance expectations of others" (p. 387). Basically, those with high EAT scores in the high goal group continued to set goals similar to the unrealistic goal set by the experimenter (i.e. 12 uses), rather than realizing that this expectation was unrealistic and setting more realistic goals. When examining goals set based on group (high, low, or self-defined) and EAT scores, it was found that those with high EAT scores in the high goal group set slightly higher goals than those with low EAT scores in the same group, individuals with high EAT scores in the low goal group set slightly lower goals than individuals with low EAT scores in the same group, and participants with high EAT scores in the self-defined goal group set much lower goals than those with low EAT scores in the same group (Pliner \& Haddock, 1996). Based on these differences, Pliner and Haddock concluded that females who were weight concerned (high EAT scores) followed more closely the goals set by others, and therefore had high amounts of maladaptive perfectionism; however, they set lower 
standards for themselves than those that were not weight concerned, demonstrating lower amounts of adaptive perfectionism. Although this shows differences in amounts of perfectionism based on high versus low EAT scores, all of the participants in this study were diagnosed with AN, so it does not give any differences between those with an eating disorder, those with symptoms of an eating disorder, and those without symptoms of an eating disorder. The experimenters also determined their own definition of perfectionism rather than administering an assessment that measured perfectionism. The differences found, however, lend support for amounts of perfectionism differing based on severity of eating problems. Pliner and Haddock also only used females in their study, and it is unclear whether these results would be replicated with males.

Bastiani et al. (1995) compared perfectionism scores in 11 females diagnosed with AN (underweight) to 8 females who had restored weight to a healthy measure, and also had a control group of 10 healthy females. The participants all completed the Eating Disorder Inventory (EDI), Hewitt Multidimensional Perfectionism Scale (Hewitt MPS), and the Frost Multidimensional Perfectionism Scale (Frost MPS) (Bastiani et al., 1995). The underweight group and weight restored group scores similarly on all of the subscales of the Frost MPS and Hewitt MPS (Bastiani et al., 1995). On the Frost MPS, Bastiani et al. found that the underweight AN participants scored higher on all of the subscales with the exception of parental expectations (PE) than the control group. On the Hewitt MPS, the underweight AN participants scored significantly higher than the control group on both self-oriented (similar to adaptive) and socially prescribed (similar to maladaptive) perfectionism, but did not differ on other-oriented perfectionism (Bastiani et al., 1995). The restored weight participants scored significantly higher than the control group on self-oriented perfectionism, but not on socially prescribed or other-oriented 
perfectionism (Bastiania et al., 1995). Going against the conclusion of Frost et al. (1990) when developing the Frost MPS, Bastiani et al. found that "anorexics experience their perfectionism as self-imposed, and not as a response to other's expectations" (p. 150). The results of the Bastiani et al. article provide further support for the relationship between perfectionism and eating disorders using a multidimensional perspective of perfectionism. However, as with most research on eating disorders, only female participants were used in this study, therefore, it is unclear if the same results would be found with male participants. Also, a discontinuum measure of eating disorders was used (EDI), and only people disagnosed with AN were participants, therefore limiting the generalizability to individuals with other diagnosable eating disorders or disordered eating behaviors.

Halmi et al. (2000) conducted a study investigating perfectionism in different subtypes of AN using 322 females diagnosed with restricting $A N(N=146)$, purging $A N(N=116)$, or binge eating and purging $\mathrm{AN}(\mathrm{N}=60)$ and also 44 comparison females that made up the control group. All participants completed the Frost Multidimensional Perfectionism Scale (Frost MPS), Eating Disorder Inventory (EDI), the Yale-Brown-Cornell Eating Disorder Scale (YBC-EDS), and an obsessive-compulsive scale (Halmi et al., 2000). Halmi et al. found that all subtypes of AN had significantly higher scores on all of the subscales of the Frost MPS with the exception of organization $(\mathrm{O})$ compared to the control group. Individuals with the three different subtypes of AN had similar scores on all of the Frost MPS subscales, with the exception of the restricting AN group having significantly lower scores than the purging subtype of AN on the parental criticism (PC) subscale (Halmi et al., 2000). Halmi et al. also found that participants' total perfectionism score on the Frost MPS was negatively correlated with lifetime lowest BMI, meaning that the lower the lowest lifetime BMI, the higher the total perfectionism score. The results demonstrated 
that when females' AN is the most severe, they have higher amounts of total perfectionism (Halmi et al., 2000). Halmi et al. concluded that "greater severity of eating disorder symptoms was associated with greater perfectionism" (p. 1804). As with the Bastiani et al. (1995) and Pliner and Haddock (1996) studies, Halmi et al. only used female participants who had been diagnosed with AN as their experimental group, limiting the generalizability of the results. However, their conclusion about perfectionism being greater as the eating disorder severity increases, lends well to studying disordered eating behaviors on a continuum in order to see the different amounts of perfectionism based on different severity of disordered eating behaviors. Even though Halmi et al. drew this conclusion, they did not use any continuum measures of disordered eating in their study, rather the conclusions were based on scores on discontinuum measures of eating disorders.

Pratt et al. (2001) researched the link between perfectionism and individuals meeting criteria for $\mathrm{BN}(\mathrm{N}=32)$, individuals classified as having $\mathrm{BED}(\mathrm{N}=127)$, and overweight individuals with no diagnosable eating disorder $(\mathrm{N}=60)$ in 219 females. The participants in the Pratt et al. study were administered the Eating Disorder Examination-Questionnaire (EDE-Q), the Questionnaire on Eating and Weight Patterns-Revised (QEWP-R), the Hewitt Multidimensional Perfectionism Scale (Hewitt MPS), the Binge Eating Scale (BES), the Symptom Checklist-90 (SCL-90), a depression inventory, and participated in a clinical interview in order to confirm diagnostic category. In the BED group, self-oriented or adaptive perfectionism was not related to any of the eating disorder measures, however socially prescribed or maladaptive perfectionism was related to three of the four EDE-Q subscales (weight concern, shape concern, and eating concern) (Pratt et al., 2001). When controlling for all other variables, it was found that the self-oriented or adaptive perfectionism score was significantly lower in the 
control group than in the $\mathrm{BN}$ or BED groups, with socially prescribed or maladaptive perfectionism being similar across the three groups (Pratt et al., 2001). In conclusion, Pratt et al. reported that self-oriented or adaptive perfectionism is not related to any eating disorder symptoms in people with BED, whereas socially prescribed or maladaptive perfectionism is associated with eating disorder symptomology in individuals with BED. However, when just examining perfectionism, self-oriented or adaptive perfectionism was the only type of perfectionism that was higher in the eating disorder groups (both BED and BN) compared to the control group (Pratt et al., 2001). The results of this study are somewhat contradictory in that adaptive perfectionism was not related to eating disorder symptoms in individuals with BED, however individuals with BED (and BN) had higher amounts of adaptive perfectionism than the control group. The results of this study are again limited in their generalizability due to using only females meeting criteria for BN or BED as the experimental group and using discontinuum measures of disordered eating; however, the results continue to shed light on the relationship between different types of perfectionism and eating disorders.

A study was conducted by Bulik et al. (2003) that investigated the relationship between eating disorders (AN and $\mathrm{BN})$ and different types of perfectionism using a nonclinical sample of female-female twin pairs. The participants in this study completed 12 questions from the Frost Multidimensional Perfectionism Scale (Frost MPS) focusing on individual rather than parental characteristics (Bulik et al., 2003). Bulik et al. also used previous interviews in order to determine diagnostic category for each participant, resulting in 34 individuals diagnosed with $\mathrm{AN}$ and 92 individuals diagnosed with $\mathrm{BN}$ for their study. Bulik et al. originally found both the concern over mistakes (CM) and doubts about actions (D) subscales of the Frost MPS, which are used to calculate maladaptive perfectionism, to be associated with both $\mathrm{AN}$ and BN; however, 
after running regression analysis, only CM and personal standards (PS) were found to be predictors of $\mathrm{AN}$ and $\mathrm{CM}$ was the only predictor of $\mathrm{BN}$. Bulik et al. concluded that "... concern over mistakes and doubts about actions appear to be the most strongly associated with the presence of eating disorders" (p. 367), which supports the notion that maladaptive perfectionism is associated with eating disorders. This finding of maladaptive perfectionism, but not adaptive perfectionism being associated with eating disorders contradicts the earlier findings of Bastiani et al. (1995) and Halmi et al. (2000). The generalizability of this study increases due to using individuals with various eating disorders; however, it is still limited by using only those diagnosed with an eating disorder compared to those with disordered eating behaviors and only females, which limits the generalizability of the findings based on sex. Given the contradictory evidence of which types of perfectionism are found to be high and/or low in an eating disorder population, it is imperative that further research be done in this area.

Ashby et al. (1998) conducted a study investigating the relationship between eating disorders and perfectionism using 24 females with a diagnosable eating disorder and 166 comparison females. All of the participants completed a demographics questionnaire, the Almost Perfect Scale-Revised, and the Frost Multidimensional Perfectionism Scale (Frost MPS), and the 24 females in the eating disorder group also completed the Eating Disorder Inventory (EDI) (Ashby et al.). Ashby et al. found that there was a significant difference between the eating disorder group and the comparison group on maladaptive perfectionism, but not on adaptive perfectionism. This is in contradiction to the findings of Bastiani et al. (1995) and Halmi et al. (2000), but supports the prediction of Frost et al. (1990) and the findings of Bulik et al. (2003) and Pliner and Haddock (1996). One of the limitations to this study was that all participants with any eating disorder (AN, BN, BED) were group together and a discontinuum measure of eating 
disorders was used, therefore, not allowing any results concerning the differences on amount of perfectionism between different disorders or any differences between those with a diagnosable eating disorder and those who partake in disordered eating behaviors. As with many of the studies reviewed, Ashby et al. also only used female participants, limiting the generalizability of the results.

Davis et al. (2000) studied the disordered eating behaviors of weight preoccupation and physical attractiveness and their relationship with perfectionism using 203 female college students. Each participant in the Davis et al. study completed three subscales of the Eating Disorder Inventory (EDI), the self-oriented perfectionism (SOP) subscale of the Hewitt Multidimensional Perfectionism Scale (Hewitt MPS), the Neurotic Perfectionism Scale (NPQ), a neuroticism subscale, and had their weight and height measured to calculate BMI. Davis et al. also had a research assistant who rated facial attractiveness (FA) on a scale from 1 to 10 , although participants were not aware that their facial attractiveness was being rated. The researchers found that neuroticism and SOP (adaptive perfectionism) impacted neurotic (maladaptive) perfectionism, while facial attractiveness, BMI, and maladaptive perfectionism all impacted weight preoccupation directly (Davis et al., 2000). Davis et al. also found that the female participants that were rated as more attractive reported more weight preoccupation than those rated less attractive. This study lends support to not only diagnosable eating disorders being related to perfectionism, but also some disordered eating behaviors being linked to perfectionism, although a discontinuum measure of eating disorders was used. As with the previous studies, however, only females were used so the results may not be generalizable to males. There must also be caution used with the results about attractiveness given the 
attractiveness rating was assigned by a research assistant, and what one person finds attractive may or may not be the same as what another person classifies as attractive.

Using 363 girls in $7^{\text {th }}$ and $8^{\text {th }}$ grade, McVey et al. (2002) conducted a study investigating risk and protective factors of disordered eating, including perfectionism. Each of the girls completed a packet of surveys including the children's version of the Eating Attitudes Test (EAT), a questionnaire titled "How Important Are These Things to How You Feel About Yourself As a Person?" that was aimed at physical appearance and social acceptance by peers, the Child and Adolescent Perfectionism scale (CAPS), several other questionnaires, and questions including describing how they felt about their body shape $(0=$ too fat, $1=$ just right, or $2=$ too thin) and answering yes or no to whether they were currently trying to lose weight (McVey et al., 2002). McVey et al. also obtained parental reports of the girls' height and weight in order to calculate BMI. Overall, McVey et al. found having low competence about physical appearance and high adaptive perfectionism to be related to disordered eating behaviors, while importance of physical appearance, having competence about social acceptance by peers, and maladaptive perfectionism were not significantly related to disordered eating behaviors. Some of the limitations involved in this study were the age of the participants and the fact that they were all females. The results were also based on the girls' current perceptions, which may change on a regular basis given the age and developmental milestones that go with this age (i.e., puberty). This is the first study reviewed that has found adaptive perfectionism to be related to disordered eating behaviors, but not maladaptive, which goes against the predictions of Frost et al. (1990) and the hypotheses of the current study. Given the data may not be generalizable to the current study population, these contradictory findings make it imperative that more research is done on 
the relationship between both adaptive and maladaptive perfectionism and disordered eating behaviors.

Hewitt et al. (1995) investigated the relationship between different types of perfectionism and various disordered eating behaviors in 81 college-aged females. The females all completed the Hewitt Multidimensional Perfectionism Scale (Hewitt MPS), the Perfectionistic SelfPresentation Scale (PSPS), the Eating Attitudes Test (EAT), the Bulimia Test (BULIT), the Body Image Avoidance Questionnaire (BIAQ), and a scale about social inadequacy (Hewitt et al., 1995). Hewitt et al. found that both adaptive and maladaptive perfectionism were related to total scores on the EAT and scores on the dieting subscale of the EAT. However, only maladaptive perfectionism was related to total scores on the BULIT, the BIAQ subscales of clothing and restraint, appearance self-esteem, and global self-esteem (Hewitt et al., 1995). With regard to the PSPS, participant scores on this were related to EAT total score, the EAT dieting subscale score, BULIT total score, the BIAQ clothing and outings subscale scores, and global self-esteem (Hewitt et al., 1995). Hewitt et al. concluded that adaptive perfectionism seemed to be related to symptoms more common in AN, whereas maladaptive perfectionism was related more broadly to disordered eating behaviors. These findings mostly agree with the predictions of Frost et al. (1990) and results of the Ashby et al. (1998), Bulik et al. (2003), and Pliner and Haddock (1996) studies. Again, only female participants were used in this study, which limits the generalizability. A strong point of this study was the various disordered eating behaviors that were examined by the researchers, however only discontinuum measures of eating disorders were used to measure these behaviors.

A study completed by Slane et al. (2010) examined the relationship between specific internalizing behaviors, such as perfectionism, and externalizing behaviors in certain disordered 
eating behaviors. The researchers used 541 participants, 335 female twins and 206 male twins from the Michigan State University Twin Registry (Slane et al., 2010). Each of the participants completed the Minnesota Eating Behavior Survey (MEBS) to measure body dissatisfaction, weight preoccupation, and binge eating; the restraint scale of the Eating Disorder ExaminationQuestionnaire (EDE-Q) to assess dietary restraint; the Frost Multidimensional Perfectionism Scale (Frost MPS); and several other measures (Slane et al., 2010). When examining the results of the Frost MPS, Slane et al. only used the concern over mistakes (CM) subscale, as a previous study using twin pairs had found CM to be the only subscale significantly associated with risk for eating disorders. Overall, Slane et al. found that females reported significantly more disordered eating behaviors than males. The researchers also found no overall difference for amount of CM between males and females (Slane et al., 2010). Slane et al. reported that all of the internalizing factors measured, including CM, were significantly related with all or most aspects of disordered eating in both males and females. In order to assess differences between males and females, Slane et al. calculated interactions between sex and each of the variables and also main effects of each of the variables. In doing this, they found a significant main effect for perfectionism when examining total MEBS score, weight preoccupation, and binge eating, where higher scores on $\mathrm{CM}$ were related to higher amounts of disordered eating, higher degrees of weight preoccupation, and higher frequency of binge eating (Slane et al., 2010). When investigating dietary restraint, Slane et al. found that those with higher BMI scores and higher amounts of perfectionism also had higher degrees of dietary restraint. Overall, the researchers concluded that there were many more similarities between males and females when examining variables associated with disordered eating behaviors (Slane et al., 2010). Specifically, they concluded that "...findings suggest that internalizing behaviors are significantly associated with 
disordered eating in both sexes..." (Slane et al., 2010, p. 156). This study provides some useful information with regards to amounts of perfectionism between males and females in association with disordered eating behaviors; however, they only used the CM subscale of the Frost instead of the entire measure. The CM subscale is used to figure both the total amount of perfectionism and the degree of maladaptive perfectionism, so the results of this study lend to amounts of maladaptive perfectionism being related to disordered eating behaviors, although that is not conclusive since results from the other three subscales that are used to calculate it were not reported.

Macedo et al. (2007) conducted a study that investigated the relationship between perfectionism and eating attitudes using 1,163 students, 764 females and 399 males, from a Portuguese university. All participants gave a self-report of height and weight and also completed the Portuguese version of the Eating Attitudes Test (EAT) and the Hewitt Multidimensional Perfectionism Scale (Hewitt MPS) (Macedo et al., 2007). When examining eating attitudes alone, Macedo et al. found that dieting concerns and total EAT scores were higher for females than for males. When examining perfectionism alone, Macedo et al. found that maladaptive perfectionism was higher in males than females. Macedo et al. found a significant relationship between total EAT scores and total Hewitt MPS scores in both males and females. Among females, Macedo et al. found dieting concerns, bulimic behaviors, and total EAT scores to be significantly related to both adaptive and maladaptive perfectionism. The results for the males were the same, with dieting concerns, bulimic behaviors, and total EAT scores all being significantly associated with both adaptive and maladaptive perfectionism (Macedo et al., 2007). In order to determine differences in amounts of perfectionism based on the degree of disordered eating behaviors, Macedo et al. distributed the participants into four groups 
based on EAT scores and then separated these into total, male, and female groups within each of the four groups, making 12 groups total. Analyses on this showed that adaptive perfectionism and total perfectionism increased as the total EAT score increased for all of the groups (Macedo et al., 2007). Further analyses demonstrated that only the group with the highest EAT score had significantly higher adaptive and total perfectionism scores than the other groups (total, male, and female), while the highest EAT score group also had higher scores on maladaptive perfectionism among total and female categories (Macedo et al., 2007). Macedo et al. concluded that "the results of our study both confirm that perfectionism is a multidimensional concept and that higher levels of perfectionism are related with abnormal EB [eating behaviors]" (p. 302). Macedo et al. also concluded that based on the results of their study, maladaptive perfectionism was related to disordered eating in both males and females, however, adaptive perfectionism was related to disordered eating in only females. This study is useful in that it uses a multidimensional model of perfectionism and studies both males and females. The authors attempted to examine eating disorders on a continuum by breaking the participants into four groups based on EAT scores; however, it is unclear if the breakdown used really demonstrates disordered eating behaviors on a continuum compared to using an actual continuum measure. This study was also completed using Portuguese university students, therefore, the results may not be generalizable to the current study population. Other limitations of the study noted by the authors were the sample of females being approximately twice that of the males and that the EAT was constructed for use with females, not males, therefore it may not be as accurate at detecting disordered eating behaviors that are common in males.

Woodside et al. (2004) investigated perfectionism and eating disorders using 42 males and 80 females from either the multisite Price Foundation Genetic Study of AN or the Price 
Foundation Genetic Study of BN. In order to obtain their sample, they used all 42 males with an eating disorder and then attempted to match each male with two females that had a similar eating disorder diagnosis (Woodside et al., 2004). Two males were only able to be matched to one female and one male was not able to be matched to any of the females, which resulted in the 80 females for the study (Woodside et al., 2004). Woodside et al. had the participants complete the Structured Interview for Anorexia Nervosa and Bulimic disorders (SIAB), the Yale-BrownCornell Eating Disorder Scale (YBC-EDS), the Frost Multidimensional Perfectionism Scale (Frost MPS), and several other measures. The goal of the researchers was "to identify characteristics that differentiate males and females with eating disorder diagnoses" (Woodside et al., 2004, p. 275). When analyzing the results from the Frost MPS, it was found that males overall scored lower on organization $(\mathrm{O})$ and concern over mistakes $(\mathrm{CM})$ compared to the females (Woodside et al., 2004). When examining sex differences by eating disorder diagnosis, Woodside et al. found that males with AN scored lower on the CM subscale of the Frost MPS compared to females with AN, while males with EDNOS had lower O subscale scores than females with EDNOS. The researchers concluded that their findings "suggest that men with ED are less perfectionistic as measured by the Frost MPS than women with ED..." (Woodside et al., 2004, p. 277). However, the only significant differences were on O, which is used to calculate total perfectionism, but is not included in adaptive or maladaptive calculations, and CM, which is used to calculate maladaptive perfectionism with three other subscales. The researchers did not calculate total, maladaptive, and adaptive perfectionism to determine if there were sex differences based on those calculations, therefore, it is unclear if males and females differ on different types of perfectionism, or only on those two subscales. Woodside et al. also used a 
clinical population, so it is not know if the same results will be found with a nonclinical population.

\section{Athletes}

As is evidenced above, the relationship between perfectionism and disordered eating behaviors is well established. However, a majority of the research investigating this link has used an eating disordered population or a nonclinical population, typically undergraduate females. A smaller number of studies have investigated specific nonclinical populations, such as athletes, when discussing eating behaviors and even fewer have examined the link between disordered eating behaviors and perfectionism in the athlete population. Some studies have found that athletes tend to have higher rates of disordered eating behaviors than non-athletes (Byrne \& McLean, 2001; Hausenblas \& Carron, 1999; Johnson et al., 1999; Petrie \& Rogers, 2001; Pritchard et al., 2007; Smolak et al., 2000; Sundgot-Borgen \& Torstveit, 2004; Szymanski \& Chrisler, 1990-1991); however, Fulkerson et al. (1999) found that athletes only have higher rates of disordered eating behaviors than non-athletes if they also have high amounts of perfectionism. Smolak et al. proposes that some of the descriptors associated with disordered eating are also associated with athletes, including perfectionism among others. There are several reasons laid out by researchers of why athletes may have higher rates of disordered eating behaviors including specific body shapes associated with specific sports, performance standards, high activity levels leading to appetite suppression, and characteristics that are descriptive of athletes being similar to those that are descriptive of eating disorders such as being perfectionistic, goaloriented, and concerned with performance (Byrne \& McLean, 2001; Hausenblas \& Carron, 1999; Petrie \& Rogers, 2001; Szymanski \& Chrisler, 1990-1991). In the below reviewed studies, the measures used to determine the association between these variables were all discontinuum 
models of disordered eating and a unidimensional perspectives of perfectionism. None of the studies used a continuum model of disordered eating or a multidimensional perspective of perfectionism, let alone both a continuum model of disordered eating and a multidimensional perspective of perfectionism. First, studies investigating the association between disordered eating behaviors and athletes will be covered, including one that examines solely females and those that compare males and females. Next, studies that investigate the link between disordered eating behaviors and perfectionism within athletes will be examined, including those that studied solely females, solely males, and those that compare males and females.

Smolak et al. (2000) completed a meta-analysis using studies that had examined eating disorders and/or disordered eating and female athletes using 34 studies. In order to be included in the meta-analysis, the studies had to have compared female athletes to a control group or use measures that could be compared to a norm (Smolak et al., 2000). Overall, Smolak et al. found female athletes to have a higher frequency of problematic eating behaviors than nonathletes. The studies that used college athletes found an even larger difference than overall, with female collegiate athletes having significantly more eating problems than female collegiate non-athletes (Smolak et al. (2000). Interestingly, Smolak et al. did not find a difference in eating behaviors between high school athletes and high school non-athletes. Smolak et al. also attempted to break the data down and found that those that participated in elite (competing at the national or international level), lean sports (e.g., dance) were more at risk for having eating problems compared to others. Based on all of the studies analyzed, Smolak et al. concluded that several different groups of athletes (different ages, sports, etc.) were at higher risk for disordered eating behaviors compared to non-athletes. The analyses of these studies lend support to athletes having higher amounts of disordered eating behaviors compared to non-athletes. As stated, this meta- 
analysis only analyzed studies using females as participants, therefore the generalizability to males is unknown. It is also unknown how disordered eating behaviors were measured in each of the studies (i.e. continuum vs. discontinuum, self-report vs. interview, etc.), which could impact the results.

Byrne and McLean (2001) conducted a review of the literature using studies that examined disordered eating and/or eating disorders in both females and ones that compared males and females. They reviewed studies that did not have a control group (uncontrolled) and studies that did have a comparison group (controlled) (Byrne \& McLean, 2001). For the uncontrolled studies that only used female participants, Byrne and McLean found a strong consensus that female athletes had high frequencies of disordered eating behaviors. However, the percentage of females engaging in these behaviors had a large range, and Byrne and McLean stated that since there was no comparison group and the measures used were all different, it was difficult to determine the accuracy of these findings. For the controlled studies using only female participants, they found mixed results, with some concluding female athletes had a higher rate of disordered eating behaviors, some concluding that disordered eating behaviors were similar between female athletes and female non-athletes, and a very few finding that female non-athletes had higher amounts of disordered eating than female athletes (Byrne \& McLean, 2001). Again, a variety of measures were used in these studies, and a variety of ages (high school, college, elite), which may lend to the inconsistencies. Byrne and McLean did state that if they separated out the studies examining elite athletes, there was a consensus of higher amounts of disordered eating behaviors among the athletes compared to the control group. Interestingly, a couple of studies reviewed by Byrne and McLean used an athlete group, a comparison group, and a clinically diagnosed eating disorder group and found that disordered eating behaviors fell on a continuum 
between these three groups with the eating disorder group having the highest amount, athletes next, and the comparison group the least amount of disordered eating behaviors. Finally, Byrne and McLean reviewed studies that compared male and female athletes to male and female comparison groups and discovered that a majority of the studies found higher amounts of disordered eating behavior among both the male and female athletes compared to same-sex comparison groups. Also, most of the studies reviewed by Byrne and McLean revealed higher amounts of disordered eating among female athletes compare to male athletes, and interestingly, one found that male athletes had higher rates of disordered eating than the female control group. This study continues to lend support to athletes having higher frequencies of disordered eating behaviors than same-sex comparison groups and female athletes having higher amounts of disordered eating behaviors than male athletes. Again, however, the studies reviewed used various measures of disordered eating behaviors and also various ages and levels of participants which could influence the results and the generalizability of the results.

Johnson et al. (1999) conducted a study investigating NCAA Division I athletes and prevalence of eating disorders. They used 1,445 varsity Division I athletes (883 males and 562 females) from 11 different schools in 11 different sports as their participants (Johnson et al., 1999). All of the participants in the Johnson et al. study completed a 133-item questionnaire which assessed eating behaviors such as binge eating, purging, dieting, body image concerns, and weight related issues, and questions assessing drug and alcohol behavior. The questionnaire also included three subscales from the Eating Disorder Inventory (EDI) (body dissatisfaction, drive for thinness, and bulimia), a self-esteem scale, and the Body Cathexis Scale (Johnson et al.). When examining binge eating, over $25 \%$ of both the male and female athletes reported binge eating at some point in their life (Johnson et al., 1999). While male athletes were more likely to 
experience an episode of overeating on a daily basis in the past three months, there was no difference found by Johnson et al. in the percentage of male and female athletes who reported ever having an overeating episode. Not surprisingly, female athletes were more likely to feel out of control when they did experience an overeating episode (Johnson et al., 1999). Using the diagnostic criteria for a binge episode (large quantity of food and feeling out of control), Johnson et al. found that female athletes were significantly more likely than male athletes to binge eat. Johnson et al. found that more female athletes had vomited and/or used laxatives to lose weight at some point in their life compared to male athletes. Female athletes were also found to have vomited more frequently on a monthly, weekly, or daily basis over the past three months compared to male athletes; however, there was no significant difference between male and female athletes when examining laxative use on a monthly, weekly, or daily basis over the past three months (Johnson et al., 1999). Females were found to have used diet pills more frequently at some point in the past and over the past three months compared to males, whereas males were more likely to use saunas or steam baths as a weight loss method (Johnson et al., 1999). Johnson et al. also analyzed the difference between self-reported fat content to ideal fat content and found that both male and female athletes wanted a fat content of below ideal, however by self-report males are at the low end of their normal level (10.5\%, normal is $10-15 \%)$ while females are below their normal level (15.4\%, normal is $19-23 \%)$. Next, Johnson et al. investigated the prevalence of clinical and subclinical eating disorder diagnoses. The researchers created four categories, including meeting DSM-IV criteria for an eating disorder (BMI = 15 or lower, amenorrhea in females, and elevation on drive for thinness and body dissatisfaction subscales of the EDI), clinically significant (same as DSM-IV except BMI = 20 or lower), self-identified, and at-risk $(\mathrm{BMI}=20$ or lower, or amenorrhea, or elevation on drive for thinness or body 
dissatisfaction subscales of the EDI) (Johnson et al., 1999). Based on these categories, no athletes met criteria for $\mathrm{AN}, 1.1 \%$ of the female athletes and no male athletes met criteria for $\mathrm{BN}$, $2.85 \%$ of the females and no males met criteria for clinically significant $\mathrm{AN}, 9.2 \%$ of females and $.005 \%$ of males met criteria for clinically significant $\mathrm{BN}, 1.96 \%$ of female athletes and $.001 \%$ of male athletes self-identified as having AN, $5.5 \%$ of females and $.005 \%$ of males selfidentified as having $\mathrm{BN}$, and $25 \%$ of female athletes and $9.5 \%$ of male athletes were categorized as at-risk for developing AN, while $38 \%$ of both females and males were considered at risk for developing BN (Johnson et al., 1999). Finally, Johnson et al. studied differences on the disordered eating measures. They found that females were significantly less satisfied with several areas of their body, as assessed with the Body Cathexis Scale, compared to males (Johnson et al., 1999). On the EDI subscales, Johnson et al. found male and female athletes to be significantly different on all three subscales, with the females scoring significantly higher than the males. Johnson et al. reported lower prevalence rates than other studies with athletes as participants; however, their opinion of this based on "postsurvey discussions with coaches, athletes, and NCAA staff is that the current results represent a conservative estimate of the extent of pathogenic eating behavior and attitudes among athletes at this level” (p. 186). The researchers believed this lower prevalence may be due to the NCAA being involved even though participants were informed that their results were confidential. The results of this study again continue to support the link between disordered eating behaviors and athletes, with higher prevalence in female athletes. However, the EDI, which was used to measure eating disorder symptoms is a discontinuum measure of eating disorders, and it is unknown if results would have been different if a continuum measure had been used. 
Sundgot-Borgen and Torstveit (2004) examined the prevalence of eating disorders in a group of 1,259 Norwegian elite athletes (572 females and 687 males) compared to 1,203 controls (574 females and 629 males). All members of the Norwegian Confederation of Sports received the initial questionnaires, which included subscales from the Eating Disorder Inventory (EDI) and questions about height and weight (Sundgot-Borgen \& Torstveit, 2004). For the control group, Sundgot-Borgen and Torstveit used a random sample, matched for age to the athletes, obtained from the Norwegian Population Register. This group also completed the initial questionnaire and then all participants (athletes and control) that were categorized as at-risk based on questionnaire answers, and a representative sample of the athletes and control group not categorized as at-risk, were interviewed by an eating disorders specialist using a format of the Eating Disorder Examination (EDE) (Sundgot-Borgen \& Torstveit, 2004). After the interview portion, 116 female athletes, 57 male athletes, 58 control females, and eight control males were categorized as at-risk for an eating disorder (Sundgot-Borgen \& Torstveit, 2004). SundgotBorgen and Torstveit's results showed that female athletes had a lower BMI than the female control group. When examining participants who met criteria for clinical and subclinical eating disorders, Sundgot-Borgen and Torstveit found that $13.5 \%$ of athletes and only $4.6 \%$ of the controls met these criteria, while $20 \%$ of the female athletes and only $8 \%$ of the male athletes met these criteria. Not surprisingly, a higher percentage of both athletes and controls met criteria for subclinical eating disorders (EDNOS or anorexia athletic (AA)) than for clinical eating disorders, which lends support to studying disordered eating behaviors on a continuum rather than a discontinuum (Sundgot-Borgen \& Torstveit, 2004). Sundgot-Borgen and Torstveit concluded that elite athletes had higher prevalence of disordered eating behaviors compared to controls and that female athletes displayed more disordered eating behaviors than male athletes. 
The findings of Sundgot-Borgen and Torstveit continue to lend support to the link between athletes and disordered eating behaviors, although they used an elite group of athletes, which may not be generalizable to collegiate athletes. Although the researchers attempted to investigate eating disorders on a continuum, they only studied the diagnosable eating disorders of AN and $\mathrm{BN}$ compared to EDNOS (still a diagnosable disorder) and AA, rather than examining other disordered eating behaviors such as dieting and weight preoccupation among others.

Pritchard et al. (2007) examined several risky health behaviors, including disordered eating behaviors, in a group of male and female athletes and also a group of male and female non-athletes. They had 506 total participants, 206 female non-athletes, 106 male non-athletes, 106 female athletes, and 88 male athletes (Pritchard et al., 2007). Each of the participants completed the Eating Attitudes Test (EAT), the Body Shape Questionnaire, and measures on alcohol consumption and smoking behaviors. Results indicated that females had greater body dissatisfaction than males, with female athletes having higher dissatisfaction than female nonathletes and male non-athletes having more dissatisfaction than male athletes (Pritchard et al., 2007). Pritchard et al. found that females engaged in more disordered eating behaviors than males and that athletes engaged in more disordered eating behaviors than non-athletes, although the difference was greater between female athletes and non-athletes than it was between male athletes and non-athletes. Pritchard et al. then categorized all participants as eating disordered or not eating disordered based on established cutoff scores for the EAT, which resulted in $13 \%$ of the female non-athletes being eating disordered, $36 \%$ of female athletes being eating disordered, $1 \%$ of the male non-athletes being eating disordered, and $9 \%$ of the male athletes being eating disordered. The differences between the athletes and non-athletes were significant for both females and males (Pritchard et al., 2007). The Pritchard et al. study continues to lend support to 
the connection between disordered eating behaviors and athletes. An interesting finding was that male non-athletes were more dissatisfied with their body image than male athletes. The current writer hypothesizes that this may be due to the male ideal body being muscular and male athletes being more likely to have obtained that ideal than male non-athletes. Also, Pritchard et al. attempted to categorize participants as eating disordered or not eating disordered, however, that is using a discontinuum model of disordered eating. For example, if the cutoff score was 20, you could have someone in the eating disordered category who scored a 21 and someone in the not eating disordered category who scored a 19. These two participants would be fairly similar, yet in different groups based on the discontinuum model. This is a main reason it is important to use a continuum model of disordered eating.

Hausenblas and Carron (1999) completed a meta-analysis using studies that examined eating disordered symptomology among athletes. In their study, Hausenblas and Carron analyzed 92 different studies, covering 10,878 athletes, which used a variety of eating disorder measures. In order to be included in the analysis, studies had to have either male or female athletes as participants (or both), have a control group or use a measure that had a normative value based on a control group, have the dependent variables be symptoms of $\mathrm{AN}, \mathrm{BN}$, or drive for thinness, and have sufficient data to calculate effect sizes in order to make equivalent comparisons (Hausenblas \& Carron, 1999). Hausenblas and Carron also coded studies based on participants' age, sex, and level of competition (high school, varsity, elite, club, and other) among other participant characteristics, and also based on whether the study was published or unpublished (e.g., dissertations) and whether there was an actual control group used in the study or the control group was based on normative data. Hausenblas and Carron then calculated effect sizes to determine the overall average effect. They found no differences between published and 
unpublished studies or between having an actual control group in the study or using normative data; therefore, all of the studies were used together in all of the analyses (Hausenblas \& Carron, 1999). Overall, Hausenblas and Carron found an effect size of .12, which is considered small, however it was significantly different from zero, therefore leading them to conclude that athletes reported more eating disorder symptoms than the control groups. Hausenblas and Carron then determined there was a difference based on sex alone (i.e., not taking into consideration athletic status), with females having a significantly smaller effect size than males (.08 and .27, respectively). Given this finding, Hausenblas and Carron analyzed male athletes and female athletes separately for the remainder of the analyses. When examining female athletes compared to controls, it was found that female athletes reported more symptoms of BN (.16) and $\mathrm{AN}(.12)$ than controls, however female athletes and controls did not differ on symptoms of drive for thinness (Hausenblas \& Carron, 1999). Hausenblas and Carron also examined differences in level of competition in females and found high school female athletes had a significantly higher effect size than club sport athletes on BN symptoms (.38 and -.11, respectively), however no differences were found in level of competition on AN symptoms or drive for thinness symptoms. For male athletes compared to male controls, Hausenblas and Carron found male athletes to report more symptoms of $\mathrm{BN}(.30), \mathrm{AN}(.35)$, and drive for thinness (.23) than controls. Hausenblas and Carron again examined the differences in level of competition and found that varsity athletes reported more symptoms of drive for thinness compared to club athletes (.49 and -.16, respectively), however no differences were found for BN symptoms and not enough studies fit into the AN category to run analyses. Interestingly, Hausenblas and Carron's results led to the conclusion that "the difference between male athletes and male controls is greater than the difference between female athletes and female controls" (p. 247), which goes against the 
findings of Pritchard et al. (2007) who found the difference between female athletes and controls to be bigger than that between male athletes and controls. In their meta-analysis, Hausenblas and Carron also found higher amounts of self-reported eating disorder symptoms among male athletes compared to female athletes, which is contradictory to the findings of Byrne and McLean (2001) and Sundgot-Borgen and Torstveit (2004) who both found higher amounts of eating disorder symptoms among female athletes. Hausenblas and Carron did caution that even though the reported symptoms of this meta-analysis were higher for males, the prevalence of eating disorders is higher in females than males. They hypothesized that this difference could be due to several reasons including differences in actual behavior and self-reported behavior, lack of information on males and eating disorder symptoms, failure to properly diagnose males, and differences in how males and females cope with eating disorders (Hausenblas \& Carron, 1999). Hausenblas and Carron found high school female athletes to have more BN symptoms and no difference between level of competition on AN or drive for thinness symptoms, which is in disagreement with the findings of Smolak et al. (2000) who found elite female athletes to have significantly more disordered eating behaviors. One of the limitations noted by Hausenblas and Carron was that most studies use either preexisting assessments or questionnaires designed by the researcher, which are both problematic. They stated that preexisting assessments are often times based on outdated DSM criteria, such as DSM-III criteria, or unable to differentiate between degrees of disorders (i.e. diagnosable, symptomatic, asymptomatic), while the measures designed by the researchers themselves lack generalizability and often times do not have accurate DSM criteria included (Hausenblas \& Carron). As their solution, Hausenblas and Carron state,

To overcome limitations of these self-report measures, researchers are encouraged to use 
the Questionnaire for Eating Disorder Diagnosis (Q-EDD; Mintz et al., 1997), which operationalizes eating disorders using the $4^{\text {th }}$ edition of the DSM and differentiates between (a) individuals with eating disorders (i.e., anorexia nervosa, bulimia nervosa, and eating disorder not otherwise specified) and individuals without eating disorders, (b) symptomatic (i.e., some eating disorder symptoms but no actual DSM-IV diagnoses) and asymptomatic (no eating disorder symptoms) and individuals with eating disorders, and (c) individuals with anorexia nervosa and individuals with bulimia nervosa (p. 249). The findings of this study give continued support to athletes having more disordered eating symptoms than non-athletes; however, the findings also shed light on some new information with regards to sex differences, differences among level of competition, and types of inventories to use, mainly using continuum measures of eating disorders such as the Q-EDD rather than discontinuum measures.

Szymanski and Chrisler (1990-1991) examined 86 college females, 66 athletes and 20 controls, in order to determine differences in degrees of disordered eating behaviors between athletes and non-athletes and also assess certain psychological variables including perfectionism. Each participant completed the Bem Sex Role Inventory (BRSI), the Eating Disorder Inventory (EDI), and answered questions about weight, height, sports team, and amount of exercise out of season (Szymanski \& Chrisler, 1990-1991). When examining the results from the EDI, athletes were found to have significantly higher scores on the bulimia, drive for thinness, ineffectiveness, interoceptive awareness, and perfectionism subscales of the EDI compared to controls, while controls had higher scores on the interpersonal distrust subscale and no differences were found between athletes and non-athletes on the body dissatisfaction or maturity fears subscales (Szymanski \& Chrisler, 1990-1991). Szymanski and Chrisler concluded that the results of their 
study "support the hypothesis that athletes have more eating pathology than non-athletes" (p. 24). This study continues to lend support to athletes having higher amounts of disordered eating behaviors than non-athletes, however there are several limitations. The researchers only used female participants; therefore, the generalizability of the results to males is unknown. They also only used the perfectionism subscale of the EDI, which is unidimensional, in order to determine the amount of perfectionism, which gives no information as to different types of perfectionism being higher or lower in athletes compared to non-athletes.

Blouin and Goldfield (1995) took a different route and only used males as participants in their study. They were investigating body image and steroid use among male bodybuilders compared to their control group of both runners and martial artists (Blouin \& Goldfield, 1995). Blouin and Goldfield had 139 participants including 43 bodybuilders, 48 runners, and 48 tae kwon do participants. In order to qualify for the study, participants had to participate in their sport at least twice per week for the past 7 months (Blouin \& Goldfield, 1995). Blouin and Goldfield also categorized their participants within each sport as competitive (training for an out of club competition or having competed in an out of club competition within the past 12 months) or recreational. Participants were administered the Eating Disorder Inventory (EDI), a modified version of the Anabolic Steroid Questionnaire (ASQ), one of three Participant Questionnaires developed by the researchers (Bodybuilding, Running, and Martial Arts), a Drive for Bulk scale that was derived as a modified version of the drive for thinness subscale of the EDI, and both a depression and self-esteem measure (Blouin \& Goldfield, 1995). Blouin and Goldfield found a significant main effect for sport on the psychological functioning variables. Specifically, Blouin and Goldfield found differences among sports on the perfectionism, ineffectiveness, and interoceptive awareness subscales of the EDI and the self-esteem measure. When examining 
perfectionism, bodybuilders had significantly higher amounts of perfectionism than runners, but not martial artists, and martial artists and runners did not differ significantly (Blouin \& Goldfield, 1995). When examining body image and eating attitudes, Blouin and Goldfield found a significant main effect for sports, with bodybuilders having higher scores on the bulimia and drive for thinness subscales of the EDI and the Drive for Bulk questionnaire compared to both runners and martial artists, and higher scores than martial artists on body dissatisfaction. Runners and martial artists did not differ significantly on any of those scales (Blouin \& Goldfield, 1995). Blouin and Goldfield concluded that bodybuilders seem to have more dissatisfaction with their bodies and higher amounts of perfectionism than martial artists and runners. Interestingly, bodybuilders scored higher on both drive for thinness and Drive for Bulk compared to martial artists and athletes, and Blouin and Goldfield hypothesized that bodybuilders need to have bulk, but it needs to be lean. Given this ideal physique for male bodybuilders, Blouin and Goldfield concluded that "in the pursuit of lean body mass, bodybuilders appear to be at risk for the development of abnormal eating practices such as binging, purging, and restricting food, as measured by the Bulimia scale of the EDI" (p. 164). Some limitations to this study are that a true control group was not used, but rather the control group was made up of athletes from other sports (i.e., running and martial arts). The results show increased disordered eating behaviors within this one subgroup of male athletes, but do not have any results that are generalizable to male athletes overall. Similar to the Szymanski \& Chrisler (1990-1991) study, a unidimensional model of perfectionism was used based off of the perfectionism subscale of the EDI. Again, this does not give information about different types of perfectionism that may differ between athletes and non-athletes. 
Fulkerson et al. (1999) conducted a study that investigated disordered eating behaviors and personality characteristics in high school athletes compared to non-athletes. As their participants, Fulkerson et al. had 309 females and 369 males, with 144 of the females being athletes and 165 non-athletes, and 174 of the males being athletes and 195 non-athletes. All of the participants were from a larger study that included an entire school district that was surveyed four years in a row (Fulkerson et al., 1999). In order to be a control participant, the person could not have participated in organized sports over the four years of the study (Fulkerson et al., 1999). Fulkerson et al. had participants complete the Eating Disorder Inventory (EDI) (bulimia, drive for thinness, body dissatisfaction, ineffectiveness, interoceptive awareness, and perfectionism subscales), Revised Restraint Scale (Restraint), DSM-III-R Eating Disorder Checklist (ED Checklist), the short form of the Multidimensional Personality Questionnaire (Negative Emotionality (NE) scale, also called Neuroticism, and Positive Emotionality (PE) scale, also called Extroversion), and obtained information on weight and height to calculate BMI. When examining the results comparing athletes and non-athletes, Fulkerson et al. found that female athletes scored lower on the ineffectiveness and interoceptive awareness subscales; however, no differences were found on the bulimia, drive for thinness, body dissatisfaction, and perfectionism subscales. There were also no significant differences between female athletes and non-athletes on the Restraint Scale, ED Checklist, and BMI calculations (Fulkerson et al., 1999). Fulkerson et al. found no significant differences between male athletes and non-athletes on any of the EDI subscales, Restraint Scale, ED Checklist, or BMI calculations. Fulkerson et al. then divided the male and female athletes into high or low perfectionism groups based on an equal split of the perfectionism subscale scores, with those having a five or less in the low perfectionism group and those having greater than five in the high perfectionism group. Based on these groupings, 
Fulkerson et al. found female athletes in the low perfectionism group had lower drive for thinness and interoceptive awareness subscale scores than those in the high perfectionism group, while there was no difference between the high and low perfectionism groups of female athletes on the Restraint Scale, ED Checklist, or BMI calculations. For male athletes, Fulkerson et al. found no significant difference between the high and low perfectionism groups on the EDI subscales; however, the high perfectionism group had significantly higher Restraint Scale scores and ED Checklist scores than the low perfectionism group. Fulkerson et al. concluded that there were no significant differences found between high school athletes and non-athletes for most of the disordered eating behaviors. Their conclusions about perfectionism were that females who had higher amounts of perfectionism would also tend to have higher scores on drive for thinness compared to those with lower amounts of perfectionism, while male athletes with higher amounts of perfectionism would be more likely to participate in restrained eating behaviors than those with lower amounts of perfectionism (Fulkerson et al., 1999). Fulkerson et al.'s final conclusion was that "it appears that adolescent athletes are not at higher risk for the development of an eating disorder than other students unless they are highly perfectionistic in their approach to sports participation and competition" (p. 78). Contrary to the other reviewed studies, Fulkerson et al. did not find athletes to have higher frequencies of disordered eating behaviors than non-athletes. However, Fulkerson et al. was using a high school population, so the results do agree with those of Smolak et al. (2000) who also found that there was no difference in disordered eating behaviors of high school athletes compared to high school non-athletes, but are still in contradiction with Hausenblas and Carron (1999) who in their analysis found that high school athletes had higher frequencies of disordered eating behaviors. A noteworthy finding by Fulkerson et al. is that athletes who have higher amounts of perfectionism also have higher 
amounts of disordered eating behaviors, which agrees with the current study hypothesis.

However, amount of perfectionism was determined by a unidimensional measure and therefore, it is unclear if different types of perfectionism varied by athletic status.

\section{Overview of the Presenting Problem, Research Questions, and Hypotheses}

The relationship between disordered eating behaviors and perfectionism has been well researched, although within the disordered eating population, there have been inconsistencies in the results when comparing males' and females' amount of perfectionism. Most studies using a multidimensional model of perfectionism have found that females with disordered eating behaviors have higher amounts of perfectionism than males with disordered eating behaviors. When studying athletes, less conclusive results have suggested that athletes may have higher levels of both disordered eating behaviors and perfectionism than non-athletes, with female athletes having higher levels of both than male athletes. However, a majority of the studies comparing male and female athletes have been conducted using a discontinuum model of eating disorders and a unidimensional model of perfectionism. Given the recent support for measuring disordered eating behaviors on a continuum rather than using specific diagnoses and support for a multidimensional model of perfectionism, it was necessary to examine the relationship between disordered eating and perfectionism comparing the two sexes (male, female) and athletic status (athlete, non-athlete) using a continuum model of disordered eating and a multidimensional model of perfectionism. The current study does exactly that; compares the frequency of disordered eating behaviors, using a continuum model, and the degree of perfectionism, using a multidimensional perspective, among male and female college athletes and non-athletes.

The following research questions will be answered in the current study. Research Question 1a: Do scores on total perfectionism vary across disordered eating categories 
(asymptomatic, symptomatic, eating disordered)? Research Question 1b: Do scores on maladaptive perfectionism vary across disordered eating categories (asymptomatic, symptomatic, eating disordered)? Research Question 1c: Do scores on adaptive perfectionism vary across disordered eating categories (asymptomatic, symptomatic, eating disordered)? Research Question 2a: Does a difference exist in the frequencies between males' and females' in the disordered eating categories (asymptomatic, symptomatic)? Research Question 2b: Does a difference exist in the frequencies between non-athletes' and athletes' in the disordered eating categories (asymptomatic, symptomatic)? Research Question 2c: Does a difference exist in the frequencies between male athletes', female athletes', male non-athletes', and female nonathletes' in the disordered eating categories (asymptomatic, symptomatic)? Research Question 3a: Are there any significant interactions on total perfectionism scores between or among the independent variables of sex, athletic status, and disordered eating category? Research Question 3b: Are there any significant interactions on maladaptive or adaptive perfectionism scores between or among the independent variables of sex, athletic status, and disordered eating category? Research Question 4a: Is there a significant difference on total perfectionism scores between males and females? Research Question $4 b$ : Is there a significant difference on maladaptive or adaptive perfectionism scores between males and females? Research Question $5 a$ : Is there a significant difference on total perfectionism scores between athletes and nonathletes? Research Question 5b: Is there a significant difference on maladaptive or adaptive perfectionism scores between athletes and non-athletes? Research Question 6a: Is there a significant difference on total perfectionism scores between the asymptomatic and symptomatic categories of disordered eating? Research Question 6b: Is there a significant difference on maladaptive or adaptive perfectionism scores between the asymptomatic and symptomatic 
categories of disordered eating? The following are the hypotheses for the above research questions.

Hypothesis 1a: Scores on total perfectionism do vary across disordered eating categories (asymptomatic, symptomatic, eating disordered), with the asymptomatic category having the lowest mean total perfectionism scores, followed by the symptomatic category, and finally the eating disordered category having the highest mean total perfectionism scores.

Hypothesis $1 b$ : Scores on maladaptive perfectionism do vary across disordered eating categories (asymptomatic, symptomatic, eating disordered), with the asymptomatic category having the lowest mean maladaptive perfectionism scores, followed by the symptomatic category, and finally the eating disordered category having the highest mean maladaptive perfectionism scores.

Hypothesis 1c: Scores on adaptive perfectionism do not vary across disordered eating categories (asymptomatic, symptomatic, eating disordered), with all three disordered eating categories (asymptomatic, symptomatic, eating disordered) having approximately the same mean adaptive perfectionism scores.

Hypothesis 2a: A difference does exist in the frequencies between males' and females' in the disordered eating categories (asymptomatic, symptomatic), with more males in the asymptomatic category, and more females in the symptomatic category.

Hypothesis 2b: A difference does exist in the frequencies between non-athletes' and athletes' in the disordered eating categories (asymptomatic, symptomatic), with more nonathletes in the asymptomatic category and more athletes in the symptomatic category.

Hypothesis 2c: A difference does exist in the frequencies between male athletes' female athletes', male non-athletes', and female non-athletes' in the disordered eating categories 
(asymptomatic, symptomatic). More specifically, there will be more male athletes than male non-athletes in the symptomatic category, more female athletes than female non-athletes in the symptomatic category, more female athletes than male athletes in the symptomatic category, and more female non-athletes than male non-athletes in the symptomatic category.

Hypothesis 3a: There are significant interactions on total perfectionism scores between or among the independent variables of sex, athletic status, and disordered eating category.

Hypothesis $3 b$ : There are significant interactions on maladaptive perfectionism scores between or among the independent variables of sex, athletic status, and disordered eating category; however, there are no significant interactions on adaptive perfectionism scores between or among the independent variables of sex, athletic status, and disordered eating category.

Hypothesis 4a: There is a significant difference on total perfectionism scores between males and females, with females having higher total perfectionism scores than males.

Hypothesis $4 b$ : There is a significant difference on maladaptive perfectionism scores between males and females, with females having higher maladaptive perfectionism scores than males; however, there is no significant difference on adaptive perfectionism scores between males and females.

Hypothesis 5a: There is a significant difference on total perfectionism scores between athletes and non-athletes, with athletes having higher total perfectionism scores than nonathletes.

Hypothesis $5 b$ : There is a significant difference on maladaptive perfectionism scores between athletes and non-athletes, with athletes having higher maladaptive perfectionism scores than non-athletes; however, there is no significant difference on adaptive perfectionism scores between athletes and non-athletes. 
Hypothesis 6a: There is a significant difference on total perfectionism scores between the asymptomatic and symptomatic categories of disordered eating, with the symptomatic category having higher total perfectionism scores than the asymptomatic category.

Hypothesis $6 b$ : There is a significant difference on maladaptive perfectionism scores between the asymptomatic and symptomatic categories of disordered eating, with the symptomatic category having higher maladaptive perfectionism scores than the asymptomatic category; however, there is no significant difference on adaptive perfectionism scores between the asymptomatic and symptomatic categories of disordered eating. 


\section{Chapter 3 - Methods}

This chapter outlines the design and methods of the study. First, the population and sample will be discussed, including how the sample was selected, the number of participants that were recruited for the study, and specific characteristics about the sample. Following this will be a description of how the data was collected, in addition to a description of each of the measures that was used. Reliability and validity information will be presented about each measure in addition to a description of the measures, including sample questions from the measures and an explanation of the scoring of each instrument. Finally, the statistical analyses that were used to test the hypotheses will be discussed.

\section{Participants}

The sample consisted of college students, including both males and females and athletes and non-athletes, at a large, private, Jesuit, Division I, Midwestern university. There were 322 participants ranging in age from 18-27, with an average age of 19.34. When considering class rank, the sample consisted of 55.28\% (178) freshman, 18.94\% (61) sophomores, $17.08 \%$ (55) juniors, and $8.7 \%$ (28) seniors. Non-athlete participants included 100 males and 101 females, and were solicited through several undergraduate courses, including those in the psychology department and Student Services department at the university. This was a convenience sampling method since professors who were willing to let their students participate determined the participants compared to using a randomized sample such as every tenth person in the student directory. The participants either signed up for a scheduled time to meet with the experimenter in a group setting with other non-athlete participants or completed the questionnaires at the end of a class period. The experiment was described to non-athlete participants during the scheduled meeting or class period and each participant decided whether to participate in the study. Some of 
the non-athlete participants received credit for participating in the study depending upon the course and professor. All of these participants were also asked if they were a member of a NCAA Division I athletic team at the university. All participants who indicated that they were a member of a NCAA team were considered athlete participants.

Sports that are offered at this university include soccer (men's and women's), basketball (women's and men's), cross country (men's and women's), golf (women's and men's), tennis (men's and women's), baseball (men's), softball (women's), crew (women's), and volleyball (women's), which are all considered non-collision sports. The athlete sample for this study was taken from the above population using a convenience cluster sampling method, meaning athletes within a cluster (i.e., a specific athletic team) were participants based on the coach's approval of meeting with the students. All of the athletic coaches were sent an email telling them about the study and asking their permission to study the athletes during a scheduled athletic meeting or practice. Based on email responses from the coaches, the athlete participants for the study were from the female volleyball, crew, soccer, and cross country teams and the male baseball and cross country teams. The researcher then scheduled a time with the coach to meet with the team, with the coach or any other athletic staff not present during the meeting. The experiment was described to team members from each team that opted to participate and each team member decided whether to participate in the study. There were 68 female athletes and 53 male athletes who participated in the study. Each athlete participant will receive one community service hour for participating in the study.

\section{Procedure}

The participants completed the two questionnaires during a scheduled time with other participants and the researcher in the room. Coaches or other athletic staff members were not 
present when the athlete participants completed the questionnaires, nor were faculty members present when the non-athlete participants completed the questionnaires. Once all of the participants were present for a scheduled session, the researcher described the study and the participants were handed a packet that included a cover letter/consent form, the Questionnaire for Eating Disorder Diagnosis (Q-EDD), and the Frost Multidimensional Perfectionism Scale (Frost MPS). The questionnaires were given to participants in a random order so that the order of assessments was not a confounding variable. Pens were also distributed to each participant. The participants were able complete both questionnaires within 25 minutes.

\section{Materials}

Questionnaire for Eating Disorder Diagnosis (Q-EDD). The participants completed the Q-EDD designed by Mintz, O’Halloran, Mulholland, and Schneider (1997) to determine their category of disordered eating (See Appendix A). The Q-EDD is a modified version of the Weight Management Questionnaire (WMQ). It is a self-report questionnaire containing 50 questions, and takes approximately five to ten minutes to complete. The questions on the Q-EDD ask about weight, height, binge eating behaviors, weight control methods, attitudes towards body shape, and menstrual cycle. For example, one question on the Q-EDD states "Do you experience recurrent episodes of binge eating, meaning eating in a discrete period of time (e.g. within any 2hour period) an amount of food that is definitely larger than most people would eat during a similar time period?" and the respondent circles Yes or No (Mintz et al.). Another question from the Q-EDD is “Does your weight and/or body shape influence how you feel about yourself?" and the respondent answers on a Likert scale of 1 (not at all) to 5 (extremely or completely) (Mintz et al., 1997). Within the Q-EDD there are instructions of how to answer all of the questions, such as "circle the answers within the two sets of [bold brackets] below that best fit for you:" (Mintz et 
al., 1997). There are also "skip" rules within the Q-EDD; for example, after the question above concerning binge-eating it states "If YES: Continue to answer the following questions. If No: Skip to Question \#4 (on the next page)" (Mintz et al., 1997).

The Questionnaire for Eating Disorder Diagnosis (Q-EDD) was scored using a manual that contains flowchart decision rules. First a BMI was calculated using the respondent's weight and height, and then the BMI was used to determine the individual's weight category (low weight, normal, overweight, moderately obese, or grossly obese). After calculating the BMI, a flowchart was followed to determine the diagnostic category. For example, one question to help determine a diagnosis of AN is "Does B.M.I. $=17.5$ or below" and the scorer circles Yes or No. There are several other questions that correlate to the diagnostic criteria for $\mathrm{AN}, \mathrm{BN}, \mathrm{BED}$, and disordered eating behaviors based on the DSM-IV, and after answering all of the relevant questions, the scorer will circle an appropriate category (asymptomatic, symptomatic, or eating disordered). For example if 'yes' was answered for all of the boxes, the diagnosis is Anorexia Nervosa. Or, if three specific criteria were yes, but the menstruating question was no, the diagnosis will be Menstruating Anorexia.

The Questionnaire for Eating Disorder Diagnosis (Q-EDD) yields both frequency data for individual behaviors and categorical labels which are used to place individuals into categories such as eating disordered or non-eating disordered (Kashubeck-West, Mintz, \& Saunders, 2001; Kashubeck-West \& Saunders, 2001; Mintz et al., 1997). The Q-EDD results in two general categories: eating disordered and non-eating disordered (Mintz et al., 1997). Both of these general categories can be broken down into more specific categories. The non-eating disordered category has two sub-categories: symptomatic and asymptomatic (Mintz et al., 1997). The eating disordered category has six sub-categories: Anorexia Nervosa (AN), Bulimia Nervosa (BN), 
subthreshold $\mathrm{BN}$, menstruating $\mathrm{AN}$, non-bingeing $\mathrm{BN}$, and binge-eating disorder (BED) (Mintz et al., 1997).

The Questionnaire for Eating Disorder Diagnosis (Q-EDD) “was developed to operationalize criteria of the DSM-IV for eating disorders" (Kashubeck-West \& Saunders, 2001, p. 63). There was no measure before the Q-EDD that distinguished between different eating disorders or that provided a differentiation between people without an eating disorder (i.e. symptomatic vs. asymptomatic). Eating disorders are of a multifaceted nature, and therefore "requires multidimensional, comprehensive assessment” (Kashubeck-West, Mintz, et al., 2001, p. 662).

The Questionnaire for Eating Disorder Diagnosis (Q-EDD) has been validated with college women, and it has also been used with high school students (Kashubeck-West, Mintz, et al., 2001). Mintz et al. (1997) conducted three studies and concluded that the psychometric properties of the Q-EDD are very good. Convergent validity was supported by the correlation between the diagnosis yielded from the Q-EDD and scores from established inventories including the Bulimia Test-Revised (BULIT-R) and Eating Attitudes Test (EAT) (Mintz et al., 1997). Criterion validity was supported due to the high correlation between the category received on the Q-EDD and diagnoses given during a clinical interview, with an accuracy rate of $98 \%$ (Mintz et al., 1997). Mintz e al. reported limited support for incremental validity for diagnosing $\mathrm{BN}$, as the Q-EDD and a clinical interview was slightly more accurate at diagnosing individuals than the BULIT-R and a clinical interview. The one significant difference reported by Mintz et al. with regards to incremental validity was the positive predictive power of the Q-EDD in diagnosing BN being .78 compared to .54 for the BULIT-R. Mintz et al. also examined the reliability of the Q-EDD and found the test-retest reliability over a 2-week period to be .94 and 
.85 for differentiation in eating disordered and non-eating disordered, and the differentiation between eating disordered, symptomatic, and asymptomatic. When assessing interrater reliability, Mintz et al. found 100\% agreement between two scorers on 50 Q-EDDs when distinguishing between eating disordered and non-eating disordered and also between eating disordered, symptomatic, and asymptomatic. Based on the support found for the psychometric properties of the Q-EDD, Kashubeck-West, Mintz, et al. (2001) concluded that "the Q-EDD is thus ideally suited to research that takes a continuum perspective on EDs" (p. 672).

The Questionnaire for Eating Disorder Diagnosis (Q-EDD) views eating disorders from the continuum perspective, or through a continuity model. Tylka and Subich (1999) found many past researchers who believed that eating disorders should be studied this way, however, other researchers believed that eating disorders should be viewed using a discontinuity model. The QEDD has healthy eating at one end (asymptomatic) and clinically diagnosable eating disorders at the other end, with various forms of disordered eating behaviors in the middle (Cohen \& Petrie, 2005; Tylka \& Subich, 1999). When disordered eating is examined along a continuum rather than people either having an eating disorder or not having an eating disorder, the differences found between groups is a matter of degree of their symptoms (Tylka \& Subic, 1999).

To support the eating disorder continuum, Tylka and Subich (1999) conducted two research studies investigating how personality, body dissatisfaction, dieting locus of control, and several psychological characteristics related to the eating disorder continuum in females. They believed if these characteristics could be placed along the eating disorder continuum, it would provide additional evidence to support the construct validity of the assessment (Tylka \& Subich, 1999). The results of Tylka and Subich's (1999) study showed several variables, such as body dissatisfaction and impulse regulation among others, which are related to eating disorders varied 
as expected along the eating disorder continuum, supporting the construct validity of the eating disorder continuum. These results provided evidence that the Q-EDD was in fact measuring the construct of disordered eating behavior, as it proposes to do, because different levels of disordered eating behaviors such as body dissatisfaction and impulse regulation varied between the categories of the Q-EDD. When comparing participants' categories on the Q-EDD and the Eating Disorder Inventory-2 (EDI-2), Tylka and Subich (1999) found that eight of the nine subscales on the EDI-2 varied based on placement into the Q-EDD categories, with perfectionism being the only one that did not differ based on Q-EDD category. Again, this supports the construct validity of the Q-EDD, specifically lending support for convergent validity, as it is correlated with another measure of eating behaviors, the EDI-2. Tylka and Subich (1999) also compared participants' scores on the Dieting Beliefs Scale (DBS) to Q-EDD category and found that the total score on the DBS did vary according to the category placement on the Q-EDD, with all three categories (asymptomatic, symptomatic, and eating disordered) significantly differing from each other. Again, this provides support for the construct validity of the Q-EDD, as it is measuring what it is supposed to measure, disordered eating behaviors, and again specifically provides evidence of convergent validity, as it is correlated with the DBS which also measures eating behaviors.

In another study by Tylka and Subich (2002), it was found that male participants' scores on three EDI-2 subscales varied according to their placement in the Q-EDD categories. They administered six of the nine subscales and found differences based on Q-EDD category on the ineffectiveness, interoceptive awareness, and maturity fears subscales, providing some additional support for the construct validity of the Q-EDD, as it is related to the construct of disordered eating behaviors, and specifically convergent validity, as it is correlated with some of the 
subscales on another measure of disordered eating behaviors, the EDI-2 (Tylka \& Subich, 2002). Tylka and Subich (2002) also found that participants' perceptions of the safety and effectiveness of weight control techniques also varied based on their placement in Q-EDD categories. For the three subscales they studied - long-term safety, long-term effectiveness, and short-term safety they found that long-term safety was different for all three categories (asymptomatic, symptomatic, eating disordered); however, for long-term effectiveness and short-term safety, the asymptomatic group differed from both the symptomatic and eating disordered group, but the symptomatic and eating disordered group did not differ from each other (Tylka \& Subich, 2002). The results from this study provide contradictory evidence for the construct validity of the QEDD with males, as it is correlated with some subscales of the EDI-2 and some aspects of safety and weight control, but not all of the subscales or aspects.

On the other side of the issue is the discontinuity model of eating disorders (Tylka \& Subich, 1999). "The discontinuity model states that the asymptomatic and symptomatic continuum groups are similar, with both groups differing from the eating disorder group" (Lowe et al., as cited in Tylka \& Subich, 1999, p. 275). Basically, this means that an individual either has a diagnosable eating disorder or has absolutely no signs of disordered eating. Contrary to this, Tylka and Subich (1999) concluded that if there was any discontinuity in their study, it was similarities between the eating disordered group and symptomatic group, with both differing from the asymptomatic group. This finding, however, presents another problem with the continuity model to an extent, as the continuity model states that all three groups should differ from the other groups. This provides some support for the construct validity of the Q-EDD, as it is showing that it measures the construct it is supposed to measure, disordered eating behaviors; however, it's convergent validity with other measures of disordered eating vary. 
Frost Multidimensional Perfectionism Scale (Frost MPS). The Frost Multidimensional Perfectionism Scale (Frost MPS), developed by Frost, Marten, Lahart, and Rosenblate (1990), was used to determine the amount of perfectionism demonstrated by each participant (See Appendix A). The questions on the Frost MPS were derived from existing perfectionism scales (Frost et al., 1990). During the pilot study, 67 items were used, and after reliability analyses, 47 items remained (Frost et al., 1990). Frost et al. then performed another study in which they correlated 47 items and came up with the existing 35-item scale. The Frost MPS examines different dimensions of perfectionism within individuals. It has six subscales: Concern Over Mistakes (CM), Personal Standards (PS), Parental Expectations (PE), Parental Criticism (PC), Doubts about Actions (D), and Organization (O). Sample questions include "If I fail at work/school, I am a failure as a person" and "I have extremely high goals."

Participants answer each statement on a five-point Likert scale, with 1 being Strongly Disagree, 2 is Disagree, 3 is Neutral, 4 is Agree, and 5 is Strongly Agree. Scores are then derived from adding up the totals for different subscales. After evaluation, the authors suggested not using the organization $(\mathrm{O})$ subscale score in determining a total Perfectionism score; therefore, the total score is found by adding up scores from the questions on the remaining five subscales (Frost et al., 1990). Bardone-Cone et al. (2007) further divided the scores from the Frost MPS into maladaptive and adaptive perfectionism. Maladaptive perfectionism is the sum of the concern over mistakes (CM), parental expectations (PE), parental criticism (PC), and doubts about actions (D) subscale questions. Adaptive perfectionism is the sum of the personal standards (PS) and organization (O) subscale questions.

Frost et al. (1990) defined each of the subscales used in the Frost MPS. The first subscale, concern over mistakes (CM), is when a person reacts negatively to making a mistake, 
or thinks he/she has failed if she/he makes a mistake (Frost et al., 1990). Frost et al. also noted that they may believe that others will think less of them or not respect them if they make a mistake. Personal standards (PS) is when an individual sets excessively high standards for him/herself and bases her/his self-worth on whether or not these standards are met (Frost et al., 1990). The third subscale, parental expectations (PE), is defined by Frost et al. as individuals believing that his/her parents set excessively high standards for her/him and expects him/her to be perfect. Parental criticism (PC) is when an individual perceives that her/his parents are, or were, overly critical of him/her (Frost et al., 1990). Frost et al. described doubts about actions (D) as an individual doubting her/his ability to accomplish a task. The last subscale, organization (O), is when the individual places extreme importance on things being in order and organized (Frost et al., 1999). Of these six dimensions, the major contributors to this area believe that parental connections (both PE and PC) are the core of perfectionism and its etiology (Hamachek, 1978; Hollender, 1965, as cited in Frost et al., 1993).

The psychometric properties of the Frost MPS have been found to be very good (Clavin et al., 1996; Frost et al., 1990; Frost et al., 1993; Parker \& Adkins, 1995). Evidence for convergent validity has been found be a few researchers (Clavin et al., 1996; Frost et al., 1990; Frost et al., 1993). Frost et al. (1993) found that the total perfectionism score on the Frost MPS was significantly correlated with the Hewitt and Flett Multidimensional Perfectionism Scale (Hewitt MPS) for both males and females. Frost et al. (1990) found the Frost MPS to be highly correlated with the Burns Perfectionism Scale, and also found significant correlations between the Frost MPS and the Self-Evaluative (SE) Scale of the Irrational Beliefs Test (IBT) and the Frost MPS and the Perfectionism Scale from the Eating Disorder Inventory (EDI). Clavin et al., using a group of males, found Frost MPS total perfectionism scores to be correlated with the 
total score on the Maudsley Obsessive-Compulsive Inventory and both the Checking and Doubting subscales of the Maudsley Obsessive-Compulsive Inventory.

Frost et al. (1990), in their first study with females, found the internal consistency of the subscales to range from .77 to $.93(\mathrm{CM}=.88, \mathrm{PS}=.83, \mathrm{PE}=.84, \mathrm{PC}=.84, \mathrm{D}=.77, \mathrm{O}=.93)$, with the reliability of the total Perfectionism Scale being .90. In their second study, also using females, Frost et al. (1990) found the overall internal consistency to be .91, with the internal consistency of the subscales ranging from .77 to $.94(\mathrm{CM}=.91, \mathrm{PS}=.81, \mathrm{PE}=.82, \mathrm{PC}=.77, \mathrm{D}$ $=.79, \mathrm{O}=.94)$. Frost et al. (1990) also found the six subscales to be highly correlated with one another, with the exception of the Organization $(\mathrm{O})$ subscale, which is not used to find the total perfectionism score. Parker and Adkins, using both females and males, found the internal consistency reliability of the total perfectionism score to be .88 with each of the subscales ranging from .57 to $.95(\mathrm{CM}=.90, \mathrm{PS}=.87, \mathrm{PE}=.57, \mathrm{PC}=.91, \mathrm{D}=.72, \mathrm{O}=.95)$. Clavin et al. found the internal consistency reliability with males to be .81 and concluded that the construct validity and reliability of the Frost MPS can be extended to males. In further support of the Frost MPS, Frost et al. (1990) found the internal consistency reliability of the Frost MPS to be .91, compared to .82 for the Burns Perfectionism Scale, .78 for the Self-Evaluative Scale of the Irrational Beliefs Test (IBT), and .70 for the Perfectionism Scale of the Eating Disorder Inventory (EDI).

Parker and Adkins (1995) also found that the final subscales determined through the factor analysis were accurate in measuring perfectionism, which confirms the construct validity of the assessment. Overall, Parker and Adkins found no differences between males and females regarding Frost MPS scores and concluded that the reliability and factor structure were good. This led them to support the use of the Frost MPS to measure the construct of perfectionism 
(Parker \& Adkins, 1995). Parker and Adkins also concluded that there were some minor concerns regarding the psychometric properties of the Frost MPS, namely the difference in their internal consistency reliability estimates of the parental expectations (PE) subscale and that found by Frost et al. (1990) and their finding of the personal standards (PS) subscale being negatively correlated to the total score. Clavin et al. also found an issue with the psychometrics of the Frost MPS. In their study, they found the Frost MPS to be "weakly contaminated by social desirability" (Clavin et al., 1996, p. 734).

\section{Data Analysis}

SPSS was used to complete all analyses of the current study. For research question 1a, an analysis of variance (ANOVA) was run to determine if total perfectionism mean scores vary across categories of disordered eating (asymptomatic, symptomatic, and eating disordered). For research question 1b, an ANOVA was also computed to determine if maladaptive perfectionism mean scores vary across disordered eating categories. And for research question 1c, another ANOVA was computed to determine if adaptive perfectionism mean scores vary across disordered eating categories. In these research questions, disordered eating category is the independent variable and perfectionism scores (total, maladaptive, or adaptive) are the dependent variables. An ANOVA was used with these research questions due to mean differences in perfectionism scores for each disordered eating category being compared (Gravetter \& Wallnau, 1996; Howell, 2002). An ANOVA was used rather than a $t$-test due to there being more than two means being compared, as there are three categories of disordered eating and each category will have a mean score for each type of perfectionism (Howell, 2002).

For research question $2 \mathrm{a}$, a chi-square test of independence was used to determine the difference between males' and females' frequencies in the categories of disordered eating 
(asymptomatic, symptomatic). Sex was the independent variable and disordered eating category was the dependent variable for this analysis. For research question $2 b$, a chi-square test of independence was computed to determine the difference between athletes' and non-athletes' frequencies in the categories of disordered eating. The variables used were athletic status and disordered eating category. For research question $2 \mathrm{c}$, another chi-square test of independence was computed in order to determine the difference between male athletes', female athletes', male non-athletes', and female non-athletes' frequencies in the disordered eating categories. Chisquares were used due to the categorical nature of the disordered eating variable (Gravetter \& Wallnau, 1996; Howell, 2002). The variable of disordered eating does not produce a numerical score, but rather places people into one of two categories; therefore, we need to study the frequency, or how many individuals are in each category, which required the use of a chi-square (Gravetter \& Wallnau, 1996; Howell, 2002).

Research question $3 \mathrm{a}$ used a $2 \times 2 \times 2$ ANOVA to investigate whether there were significant interactions on total perfectionism scores between or among sex, athletic status, and disordered eating category. For this question, sex, athletic status, and disordered eating category were the independent variables and total perfectionism was the dependent variable. An ANOVA was used for this research question because we were examining the "interacting effects of two or more variables" (Howell, 2002, p. 320).

For research question 3b, a 2x2x2 multivariate analysis of variance (MANOVA) was computed to determine whether there were significant interactions on maladaptive and adaptive perfectionism scores between or among sex, athletic status, and disordered eating category. Sex, athletic status, and disordered eating category served as the independent variables and maladaptive perfectionism and adaptive perfectionism served as the dependent variables. For this 
research question regarding interactions, a MANOVA was used rather than an ANOVA because there was more than one dependent variable, which required the use of a MANOVA (Tabachnick \& Fidell, 2001).

For research questions $4 \mathrm{a}, 5 \mathrm{a}$, and $6 \mathrm{a}$, one $2 \times 2 \times 2$ ANOVA was computed to determine if there were significant differences on total perfectionism scores between males and females (4a), athletes and non-athletes (5a), and male athletes, female athletes, male non-athletes, and female non-athletes (6a). Sex (4a), athletic status (5a), and sex/athletic status combination (6a) were the independent variables while total perfectionism was the dependent variable for all three questions. ANOVAs are used to find differences between means (Howell, 2002). An ANOVA was used with these research questions due to mean differences in total perfectionism scores for males and females (4a), athletes and non-athletes (5a), and male athletes, female athletes, male non-athletes, and female non-athletes (6a) being compared (Gravetter \& Wallnau, 1996; Howell, 2002).

Research questions $4 \mathrm{~b}, 5 \mathrm{~b}$, and $6 \mathrm{~b}$ were analyzed using one $2 \times 2 \times 2$ MANOVA to determine if there were significant differences on maladaptive or adaptive perfectionism between males and females (4b), athletes and non-athletes (5b), and male athletes, female athletes, male non-athletes, and female non-athletes (6b). For research question $4 b$, sex was the independent variable, for research question $5 b$, athletic status was the independent variable, and for research question 6b, sex/athletic status combined was the independent variable. Maladaptive perfectionism and adaptive perfectionism were the dependent variables in all three questions. Similar to ANOVAs, MANOVAs are used to find mean differences between groups (Tabachnick \& Fidell, 2001). A MANOVA was used for this question due to having more than one dependent variable: maladaptive perfectionism and adaptive perfectionism (Tabachnick \& Fidell, 2001). 
Tabachnick and Fidell report that the "minimum data set for a MANOVA has one or more IVs, each with two or more levels, and two or more DVs for each subject within each combination of IVs" (p. 332).

\section{Summary}

In summary, 201 college non-athletes (100 males and 101 females) and 121 collegiate athletes (68 females and 53 males) participated in the study. The non-athlete participants were solicited through various undergraduate courses at a large Midwestern university, which resulted in a convenience sample. The athlete participants were solicited through the athletic department of the same large Midwestern university, by communication with coaches, which resulted in a convenience cluster sample based on coaches' approval of the researcher attending a team meeting or practice to administer the assessments. Each participant that agreed to participate received a verbal description of the study from the researcher. After hearing the description of the study, those willing to participate completed the Questionnaire for Eating Disorder Diagnosis (Q-EDD) and Frost Multidimensional Perfectionism Scale (Frost MPS), in random order, in a group setting. The data was entered into SPSS and analyses were run to test the three research questions and hypotheses. To determine if perfectionism (total, adaptive, and maladaptive) scores vary based on disordered eating category among athletes and among non-athletes, two 3x3 ANOVAs were run. A chi-square test of independence with a $3 \times 2 \times 2$ contingency table was used to determine the difference between male athletes' and female athletes', female non-athletes' and male non-athletes', male athletes' and male non-athletes', and female athletes' and female nonathletes' placement into one of the three categories of disordered eating (asymptomatic, symptomatic, eating disordered). Four 2x3 MANOVAs were used to determine if there is a difference on total, adaptive, and maladaptive perfectionism scores between male athletes and 
female athletes, male non-athletes and female non-athletes, female athletes and female nonathletes, and male athletes and male non-athletes. 


\section{Chapter 4 - Results}

The following chapter explains and summarizes the statistical analyses used to test the research questions and hypotheses for the current study. First, an overview of the participant characteristics will be presented. Next, research questions 1a, 1b, and 1c will be addressed including a brief description of the statistical analyses used for these questions and the results of the statistical analyses. Following this, research questions $2 \mathrm{a}, 2 \mathrm{~b}$, and $2 \mathrm{c}$ will be discussed. Again, a brief description of the statistical analyses and the results of the statistical analyses will be given. The same will be done for research questions $3 a$ and $3 b, 4 a$ and $4 b, 5 a$ and $5 b$, and $6 a$ and $6 \mathrm{~b}$; a brief description of the statistical analyses followed by the results of the statistical analyses. Finally, a summary of the results will be presented.

\section{Participant Characteristics}

As part of the Questionnaire for Eating Disorder Diagnosis (Q-EDD), participants were asked their age, class status (i.e., freshman, sophomore, etc.), and race. Over $50 \%$ of the sample was between 18-19 years of age while less than $50 \%$ were all other ages combined. The average age for the total sample was 19.34, with ages ranging from 18 to 27 (Table 1). The male nonathletes had an average age of 19.43 , the female non-athletes had an average age of 19.25 , the male athletes had an average age of 19.43, and the female athletes had an average age of 19.56.

When looking at class status, over $50 \%$ of participants identified themselves as freshmen, while less than 50\% identified as sophomores, juniors, and seniors combined (Table 2). Sixtyseven $(67 \%)$ of the male non-athletes were freshman, $16(16 \%)$ were sophomores, $12(12 \%)$ were juniors, and five (5\%) were seniors. For the female non-athletes, $58(57.43 \%)$ identified as freshman, $18(17.82 \%)$ identified as sophomores, $16(15.84 \%)$ identified as juniors, and nine $(8.91 \%)$ identified as seniors. Twenty-four $(45.28 \%)$ of the male athletes were freshman, 13 
(24.53\%) were sophomores, $13(24.53 \%)$ were juniors, and three (5.66\%) were seniors. Finally, for the female athletes, 29 (42.65\%) classified as freshman, 14 (20.59\%) classified as sophomores, $14(20.59 \%)$ classified as juniors, and $11(16.18 \%)$ classified as seniors.

Two hundred fifty-three participants (81.6\%) identified as Caucasian/White (Table 3). The racial identifications were more diverse for the non-athletes than they were for the athletes, as over $93 \%$ of the athletes identified as Caucasian/White (Tables 4-5).

Table 1.

Age

\begin{tabular}{|c|c|c|c|c|}
\hline \multicolumn{5}{|c|}{ Age } \\
\hline & & & & Cumulative \\
\hline & & Frequency & Percent & Percent \\
\hline \multirow[t]{8}{*}{ Years } & 18 & 77 & 23.91 & 23.91 \\
\hline & 19 & 136 & 42.23 & 66.14 \\
\hline & 20 & 58 & 18.01 & 84.15 \\
\hline & 21 & 31 & 9.63 & 93.78 \\
\hline & 22 & 17 & 5.28 & 99.06 \\
\hline & 23 & 2 & .62 & 99.68 \\
\hline & 27 & 1 & .32 & 100.00 \\
\hline & Total & 322 & 100.00 & \\
\hline Missing & System & 0 & 0 & \\
\hline Total & & 322 & 100.00 & \\
\hline
\end{tabular}


Table 2.

Class Status

\section{Class Status}

\begin{tabular}{llccc}
\hline \hline & & & & Cumulative \\
& & Frequency & Percent & Percent \\
\hline & Freshman & 178 & 55.28 & 55.28 \\
& Sophomore & 61 & 18.94 & 74.22 \\
& Junior & 55 & 17.08 & 91.3 \\
& Senior & 28 & 8.70 & 100.00 \\
Missing & Total & 322 & 100.00 & \\
Total & System & 0 & 0 & \\
\hline \hline
\end{tabular}


Table 3.

Race

\section{Race}

\begin{tabular}{|c|c|c|c|c|}
\hline & & \multicolumn{3}{|r|}{$\overline{\text { Cumulative }}$} \\
\hline & & Frequency & Percent & Percent \\
\hline & Caucasian/White & 262 & 81.36 & 81.36 \\
\hline & Asian American/ & 23 & 7.14 & 88.50 \\
\hline & Pacific Islander & & & \\
\hline & Hispanic/Latino/ & 13 & 4.04 & 92.54 \\
\hline & Mexican-American & & & \\
\hline & African-American/Black & 7 & 2.17 & 94.71 \\
\hline & American Indian & 3 & .93 & 95.64 \\
\hline & Other & 14 & 4.36 & 100.0 \\
\hline & Total & 322 & 100.0 & \\
\hline Missing & System & 0 & 0 & \\
\hline Total & & 322 & 100.0 & \\
\hline
\end{tabular}


Table 4.

Non-Athlete Race

\section{Race}

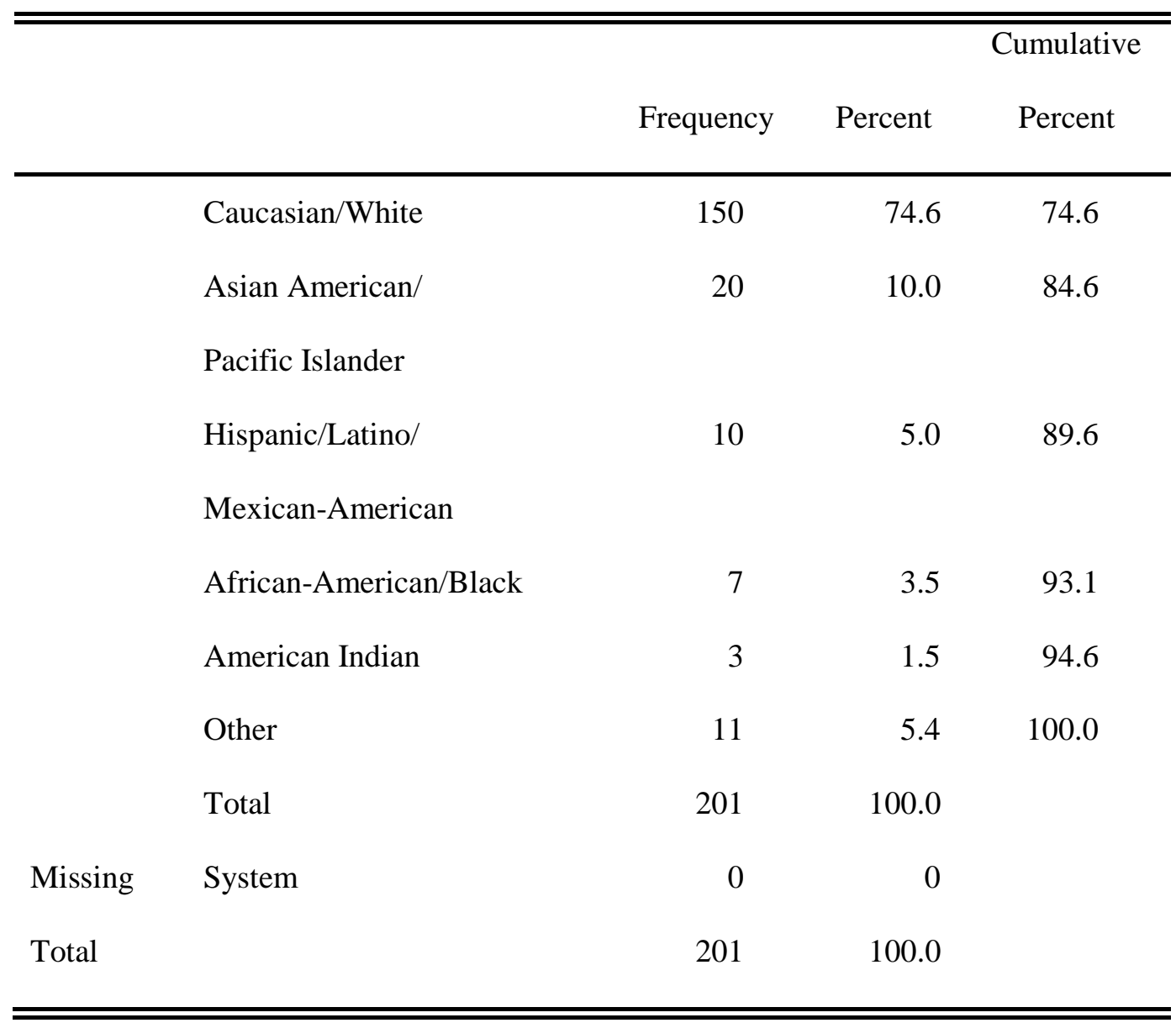


Table 5 .

Athlete Race

\begin{tabular}{|c|c|c|c|c|}
\hline \multicolumn{5}{|c|}{ Race } \\
\hline & & & & \multirow{2}{*}{$\begin{array}{c}\text { Cumulative } \\
\text { Percent }\end{array}$} \\
\hline & & Frequency & Percent & \\
\hline & Caucasian/White & 112 & 92.5 & 92.6 \\
\hline & Asian American/ & 3 & 2.5 & 95.1 \\
\hline & \multicolumn{4}{|l|}{ Pacific Islander } \\
\hline & Hispanic/Latino/ & 3 & 2.5 & 97.6 \\
\hline & \multicolumn{4}{|l|}{ Mexican-American } \\
\hline & African-American/Black & 0 & 0.0 & 97.6 \\
\hline & American Indian & 0 & 0.0 & 97.6 \\
\hline & Other & 3 & 2.5 & 100.0 \\
\hline & Total & 121 & 100.0 & \\
\hline Missing & System & 0 & 0 & \\
\hline Total & & 121 & 100.0 & \\
\hline
\end{tabular}

\section{Results of Statistical Analyses}

Six research questions were proposed in the current study based on previous literature and findings. For research questions 1a, 1b, and 1c, all 322 original participants were used in the data analyses; however, due to the low number of individuals classified as eating disordered $(\mathrm{N}=12)$, all other analyses only included the participants classified as asymptomatic and symptomatic $(\mathrm{N}=310)$. The research questions focus on whether perfectionism scores (total, maladaptive, and adaptive) vary based on disordered eating category (asymptomatic, 
symptomatic, and eating disordered), whether the different groups of participants (i.e., athletes vs. non-athletes, males vs. females, and male athletes vs. female athletes vs. male non-athletes vs. female non-athletes) differ on their placement into the two categories of disordered eating (asymptomatic and symptomatic), whether there are significant interactions on perfectionism (total, adaptive, maladaptive) among the independent variables of sex, athletic status, and disordered eating category, and whether there are differences in amounts of perfectionism (total, adaptive, maladaptive) between males and females, athletes, and non-athletes, and asymptomatic and symptomatic individuals.

Research Question 1a: Do scores on total perfectionism vary across disordered eating categories (asymptomatic, symptomatic, eating disordered)?

An ANOVA was computed to test the hypothesis that scores on total perfectionism vary across disordered eating category. For this analysis, diagnostic category was entered as the fixed independent variable and total perfectionism was entered as the dependent variable. An alpha level of .025 was used in order to keep the experimentwise error to an acceptable level. In support of the hypothesis, a significant difference was found when analyzing total perfectionism based on disordered eating category, $\mathrm{F}(2,319)=12.18, p=<.01$ (See Tables 6 and 7). Table 6.

Descriptive Statistics for Total Perfectionism and Disordered Eating Descriptive Statistics for Total Perfectionism

\begin{tabular}{lccccccc}
\hline \hline & \multicolumn{2}{c}{ Asymptomatic } & & \multicolumn{2}{c}{ Symptomatic } & & \multicolumn{2}{c}{ Eating Disordered } \\
& $M$ & Std. Error & $M$ & Std. Error & $M$ & Std. Error \\
& & & & & & \\
\hline Total Perfectionism & 80.96 & 1.06 & 89.64 & 1.69 & 94.25 & 4.56 \\
\hline \hline
\end{tabular}


Table 7.

Summary of Total Perfectionism and Disordered Eating

Summary of Total Perfectionism

\begin{tabular}{llllll}
\hline \hline & $\begin{array}{c}\text { Sum of } \\
\text { Squares }\end{array}$ & $d f$ & $\begin{array}{c}\text { Mean } \\
\text { Square }\end{array}$ & F & $p$ \\
\hline Total Perfectionism & 6077.44 & 2 & 3038.72 & 12.18 & $<.01$ \\
& & & & & \\
\hline \hline
\end{tabular}

Due to the significant difference on total perfectionism across the disordered eating categories, pairwise comparisons were computed to determine which categories were significantly different from one another. Results indicated a significant difference between the asymptomatic and symptomatic categories, $p=<.01$, and a significant difference between the asymptomatic and eating disordered category, $p=.01$. This finding supports the hypotheses that total perfectionism would vary across disordered eating categories. An explanation for the support of this hypothesis will be offered in the next chapter. See Table 8 and Graph 1 for a summary of this analysis.

Table 8 .

Pairwise Comparison for Total Perfectionism based on Disordered Eating Category Pairwise Comparisons for Total Perfectionism

\begin{tabular}{ccccc}
\hline \hline & & \multicolumn{3}{c}{ Total } \\
(I)Diagnostic & $(\mathrm{J})$ Diagnostic & Mean & Std. & $p$ \\
Category & Category & Diff. & Error & \\
\hline Asymptomatic & Symptomatic & -8.68 & 2.00 & $<.01$ \\
& Eating Disordered & -13.29 & 4.68 & .01 \\
Symptomatic & Eating Disordered & -4.61 & 4.86 & 1.00 \\
\hline \hline
\end{tabular}


Figure 1.

Total Perfectionism Means

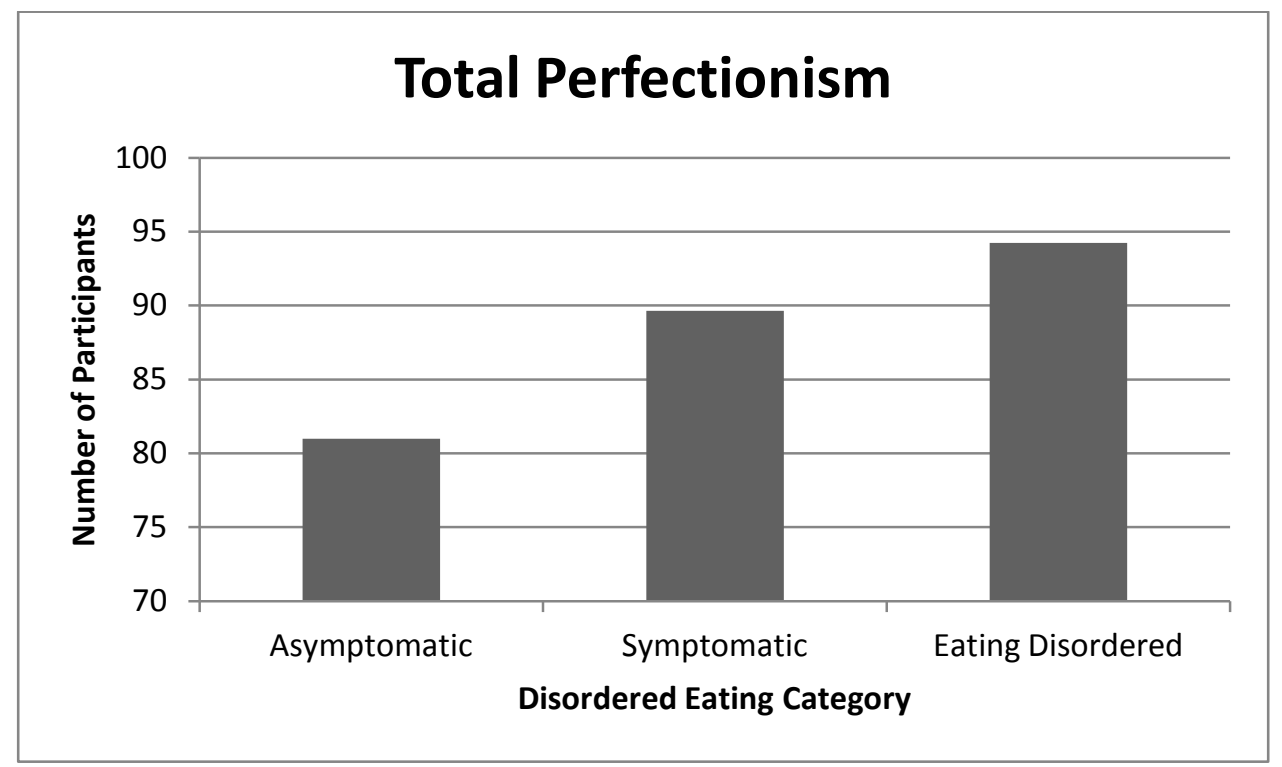

Research Question 1b: Do scores on maladaptive perfectionism vary across disordered eating categories (asymptomatic, symptomatic, eating disordered)?

For research question $1 \mathrm{~b}$, an ANOVA was computed to test the hypothesis that scores on maladaptive perfectionism vary across disordered eating categories. Diagnostic category was entered as the fixed independent variable and maladaptive perfectionism was entered as the dependent variable in SPSS. An alpha level of .025 was used in order to keep the experimentwise error rate to an acceptable level. In support of the hypothesis, a significant difference was found when analyzing maladaptive perfectionism based on disordered eating category, $\mathrm{F}(2,319)=$ $15.19, p=<.01$ (See Tables 9 and 10). 
Table 9.

Descriptive Statistics for Maladaptive Perfectionism and Disordered Eating

Descriptive Statistics for Maladaptive Perfectionism

\begin{tabular}{lcccccc}
\hline \hline & \multicolumn{2}{c}{ Asymptomatic } & & \multicolumn{2}{c}{ Symptomatic } & \multicolumn{2}{c}{ Eating Disordered } \\
& $M$ & Std. Error & $M$ & Std. Error & $M$ & Std. Error \\
& & & & & & \\
\hline Maladaptive & 55.77 & .87 & 64.10 & 1.40 & 66.50 & 3.76 \\
Perfectionism & & & & & & \\
\end{tabular}

Table 10.

Summary of Maladaptive Perfectionism and Disordered Eating

Summary of Maladaptive Perfectionism

\begin{tabular}{lllllc}
\hline \hline & $\begin{array}{c}\text { Sum of } \\
\text { Squares }\end{array}$ & $d f$ & $\begin{array}{c}\text { Mean } \\
\text { Square }\end{array}$ & F & $p$ \\
\hline Maladaptive Perfectionism & 5149.83 & 2 & 2574.91 & 15.19 & $<.01$ \\
\hline \hline
\end{tabular}

Due to the significant difference on maladaptive perfectionism across the disordered eating categories, a pairwise comparison was computed to determine which categories were significantly different from one another. Results revealed a significant difference between the asymptomatic and symptomatic categories, $p=<.01$, and a significant difference between the asymptomatic and eating disordered categories, $p=.02$. This finding supports the hypothesis that maladaptive perfectionism would vary across disordered eating categories. An explanation for the support of this hypothesis will be offered in the next chapter. A summary of these analyses can be found in Table 11 and Graph 2. 
Table 11.

Pairwise Comparison for Maladaptive Perfectionism based on Disordered Eating Category Pairwise Comparisons for Maladaptive Perfectionism

\begin{tabular}{ccccc}
\hline \hline & & \multicolumn{3}{c}{ Maladaptive } \\
(I)Diagnostic & $(\mathrm{J})$ Diagnostic & Mean & Std. & $p$ \\
Category & Category & Diff. & Error & \\
\hline Asymptomatic & Symptomatic & -8.32 & 1.65 & $<.01$ \\
& Eating Disordered & -10.73 & 3.86 & .02 \\
Symptomatic & Eating Disordered & -2.40 & 4.01 & 1.00 \\
\hline \hline
\end{tabular}

Figure 2.

Maladaptive Perfectionism Means

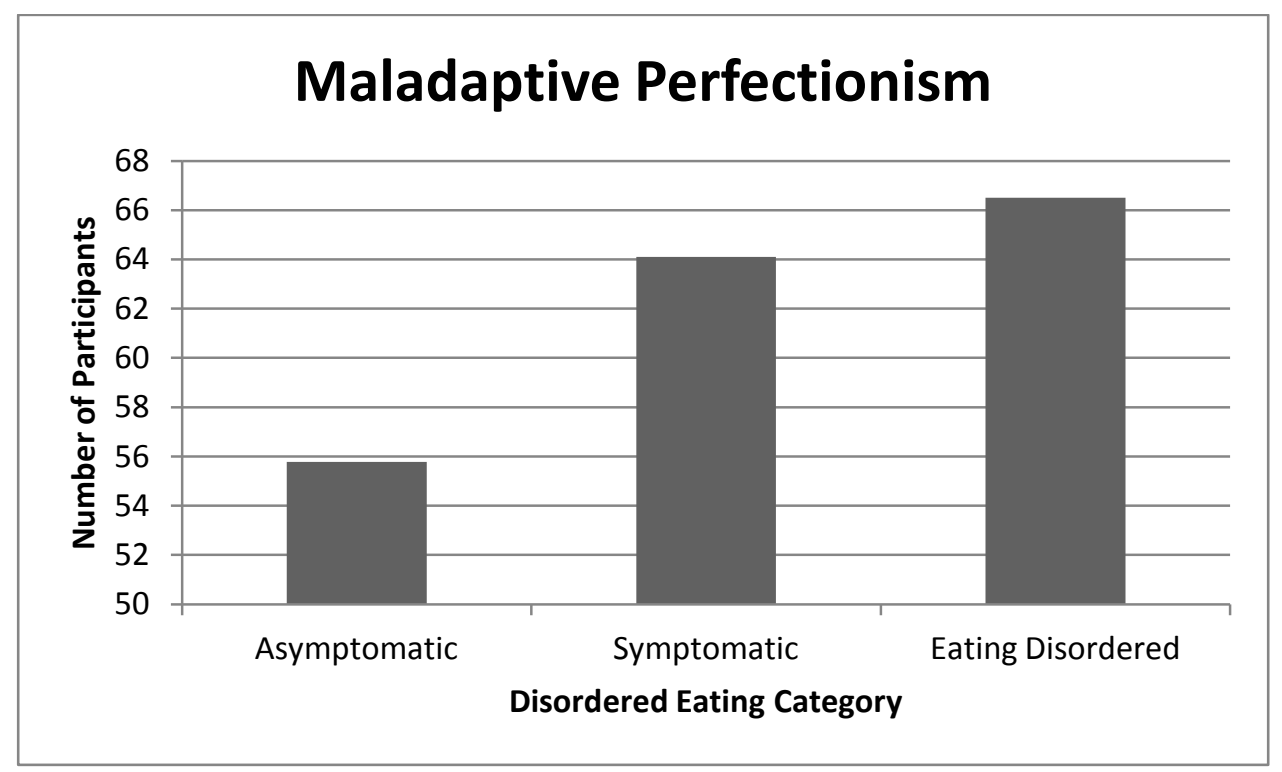


Research Question 1c: Do scores on adaptive perfectionism vary across disordered eating categories (asymptomatic, symptomatic, eating disordered)?

For this research question, an ANOVA was computed to test the hypothesis that scores on adaptive perfectionism do not vary across disordered eating categories. For this analysis, diagnostic category was entered as the fixed independent variable and adaptive perfectionism was entered as the dependent variable. An alpha level of .025 was used in order to keep the experimentwise error rate to an acceptable level. There was no significant difference found when analyzing adaptive perfectionism based on disordered eating category, $\mathrm{F}(2,319)=.08, p=.93$, which supports the hypotheses. These analyses are summarized in Tables 12 and 13 and Graph 3.

\section{Table 12.}

Descriptive Statistics for Adaptive Perfectionism and Disordered Eating

Descriptive Statistics for Adaptive Perfectionism

\begin{tabular}{|c|c|c|c|c|c|c|}
\hline & \multicolumn{2}{|c|}{ Asymptomatic } & \multicolumn{2}{|c|}{ Symptomatic } & \multicolumn{2}{|c|}{ Eating Disordered } \\
\hline & $M$ & Std. Error & $M$ & Std. Error & $M$ & Std. Error \\
\hline Adaptive & 48.27 & .75 & 49.24 & 1.12 & 40.00 & 4.82 \\
\hline Perfectionism & & & & & & \\
\hline
\end{tabular}


Table 13.

Summary of Adaptive Perfectionism and Disordered Eating

Summary of Adaptive Perfectionism

\begin{tabular}{lrrrrr}
\hline \hline & $\begin{array}{c}\text { Sum of } \\
\text { Squares }\end{array}$ & $d f$ & $\begin{array}{c}\text { Mean } \\
\text { Square }\end{array}$ & F & $p$ \\
\hline Adaptive Perfectionism & 8.21 & 2 & 4.11 & .08 & .93 \\
\hline \hline
\end{tabular}

Figure 3.

\section{Adaptive Perfectionism Means}

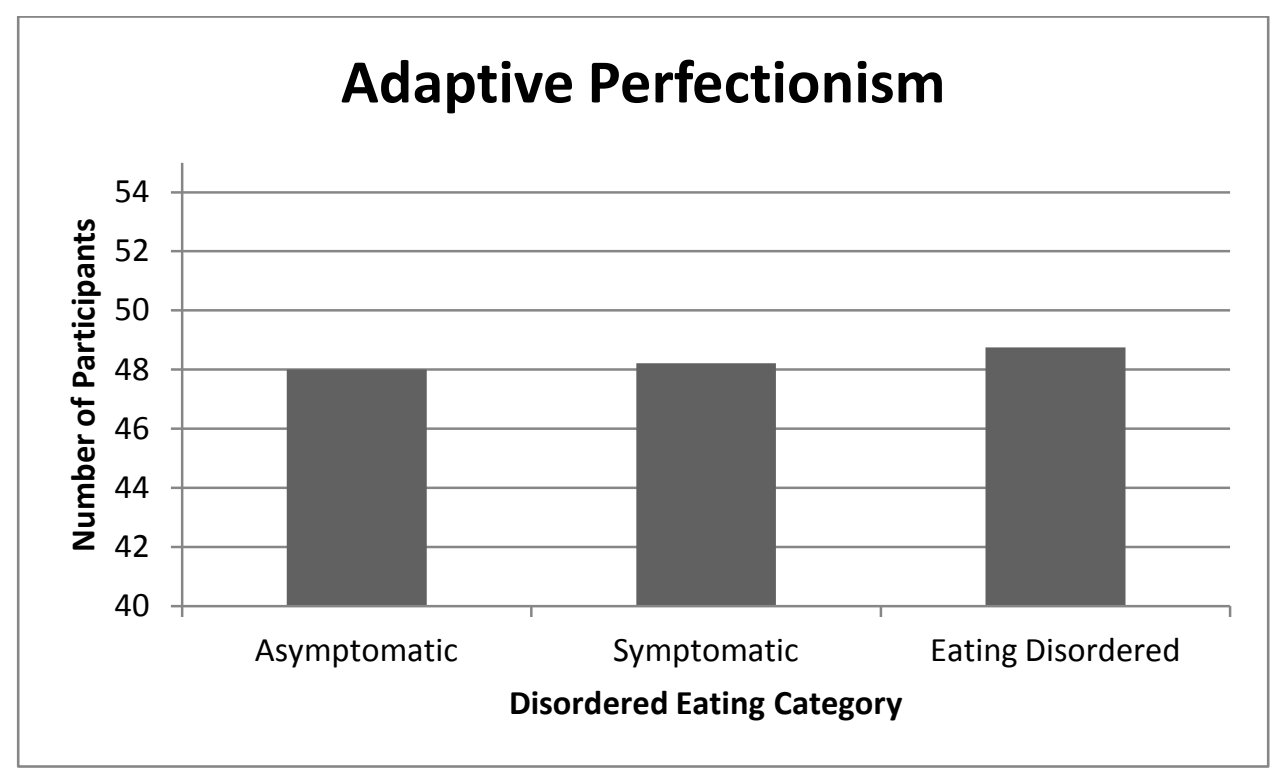

Research Question 2a: Does a difference exist in the frequencies between males' and females' in the disordered eating categories (asymptomatic, symptomatic)?

For this research question, a chi-square test of independence with crosstabs was used in SPSS to determine if the frequencies in each disordered eating category differed between males and females. An alpha level of .05 was used for this research question. In support of the hypothesis, results revealed a significant difference in the frequencies between males' and females' in the two disordered eating categories, $\chi^{2}(1,308)=5.29, p=.02$. There were more 
males than expected in the asymptomatic category and there were more females than expected in the symptomatic category. See Tables 14 and 15 for a summary of this analysis. An explanation for the result will be offered in the following chapter.

Table 14.

Descriptive Data for Chi-Square for Sex and Disordered Eating Category

Descriptive Data for Sex

\begin{tabular}{lllccc}
\hline \hline & & & Sex & \\
& & & Males & Females & Total \\
\hline Category & Asymptomatic & Count & 117.0 & 106.0 & 223.0 \\
& & Expected Count & 107.9 & 115.1 & 223.0 \\
& Symptomatic & Count & 33.0 & 54.0 & 87.0 \\
& & Expected Count & 42.1 & 44.9 & 87.0 \\
& & Count & 15.0 & 160.0 & 310.0 \\
& & Expected Count & 150.0 & 160.0 & 310.0 \\
\hline \hline
\end{tabular}

Table 15.

Chi-Square Results for Sex and Disordered Eating Category

Chi-Square for Sex

\begin{tabular}{llll}
\hline \hline & Value & df & $p$ \\
\hline Pearson Chi-Square & 5.29 & 1 & .02 \\
\hline \hline
\end{tabular}

Research Question 2b: Does a difference exist in the frequencies between non-athletes' and athletes' in the disordered eating categories (asymptomatic, symptomatic)? 
A chi-square test of independence with crosstabs was used to determine if the frequencies between non-athletes and athletes in the disordered eating categories significantly differed. An alpha level of .05 was used for this research question. Results did not indicate a significant difference in the frequencies between athletes' and non-athletes' in the two categories of disordered eating, $\chi^{2}(1,308)=.88, p=.35$, which did not support the hypothesis. The results of this analysis are summarized in Tables 16 and 17. An explanation for this hypothesis not being supported will be offered in the following chapter.

Table 16.

Descriptive Data for Chi-Square for Athletic Status and Disordered Eating Category

Descriptive Data for Athletic Status

\begin{tabular}{|c|c|c|c|c|c|}
\hline & & \multicolumn{4}{|c|}{ " Athletic Status (AS) } \\
\hline & & & Athlete & Non-Athlete & Total \\
\hline \multirow[t]{4}{*}{ Category } & Asymptomatic & Count & 82.0 & 141.0 & 223.0 \\
\hline & & Expected Count & 85.6 & 137.4 & 223.0 \\
\hline & Symptomatic & Count & 37.0 & 50.0 & 87.0 \\
\hline & & Expected Count & 33.4 & 53.6 & 87.0 \\
\hline \multirow[t]{2}{*}{ Total } & & Count & 119.0 & 191.0 & 310.0 \\
\hline & & Expected Count & 119.0 & 191.0 & 310.0 \\
\hline
\end{tabular}


Table 17.

Chi-Square Results for Athletic Status and Disordered Eating Category

Chi-Square for Athletic Status

\begin{tabular}{lccc}
\hline \hline & Value & $\mathrm{df}$ & $p$ \\
\hline Pearson Chi-Square & .88 & 1 & .35 \\
& & & \\
\hline \hline
\end{tabular}

Research Question 2c: Does a difference exist in the frequencies between male athletes', female athletes', male non-athletes', and female non-athletes' in the disordered eating categories (asymptomatic, symptomatic)?

For research question 2c, a chi-square test of independence with crosstabs was used in SPSS to determine if the frequencies differed between the groups above in the disordered eating categories. For this research question, an alpha level of .05 was used. Results did not indicate a significant difference in the frequencies between male athletes', female athletes', male nonathletes, and female non-athletes' in the two categories of disordered eating, $\chi^{2}(1,308)=7.19, p$ $=.07$, which did not support the hypothesis. Tables 18 and 19 summarize the findings from this analysis. An explanation for the hypothesis not being supported will be given in the following chapter. 
Table 18.

Descriptive Data for Chi-Square for Sex/Athletic Status in Disordered Eating Category

Descriptive Data for Sex/Athletic Status

\begin{tabular}{|c|c|c|c|c|c|c|c|}
\hline & & \multicolumn{6}{|c|}{ Sex/Athletic Status (SAS) } \\
\hline & & & MA & MNA & FA & FNA & Total \\
\hline \multirow[t]{6}{*}{ Category } & Asymptomatic & Count & 37.0 & 80.0 & 45.0 & 61.0 & 223.0 \\
\hline & & Expected & 37.4 & 70.5 & 48.2 & 66.9 & 223.0 \\
\hline & & Count & & & & & \\
\hline & Symptomatic & Count & 15.0 & 18.0 & 22.0 & 32.0 & 87.0 \\
\hline & & Expected & 14.6 & 27.5 & 18.8 & 26.1 & \\
\hline & & Count & & & & & \\
\hline \multirow[t]{3}{*}{ Total } & & Count & 52.0 & 98.0 & 67.0 & 93.0 & 310.0 \\
\hline & & Expected & 52.0 & 98.0 & 67.0 & 93.0 & 310.0 \\
\hline & & Count & & & & & \\
\hline
\end{tabular}

Table 19.

Chi-Square Results for Sex/Athletic Status and Disordered Eating Category

Chi-Square for Sex/Athletic Status

\begin{tabular}{llll}
\hline \hline & Value & df & $p$ \\
\hline Pearson Chi-Square & 7.19 & 3 & .07 \\
\hline \hline
\end{tabular}


Research Question 3a: Are there any significant interactions on total perfectionism scores between or among the independent variables of sex, athletic status, and disordered eating category?

For research question 3a, a 2x2x2 ANOVA was computed to determine if the hypothesis that there would be significant interactions on total perfectionism scores between or among the three independent variables of sex, athletic status, and disordered eating category was supported. An alpha level of .025 was used for this analysis in order to keep the experimentwise error rate to an acceptable level. Results indicated no significant interaction on total perfectionism between sex and athletic status, $\mathrm{F}(1,302)=.09, p=.77$, between sex and disordered eating category, $\mathrm{F}(1,302)=4.25, p=.04$, between athletic status and disordered eating category, $\mathrm{F}(1,302)=.04, p$ $=.85$, or among sex, athletic status, and disordered eating category, $\mathrm{F}(1,302)=.73, p=.39$, which did not support the hypothesis (See Table 20). An explanation for this hypothesis not being supported with be discussed in the following chapter.

Table 20.

Summary of Total Perfectionism Interactions

Summary for Total Perfectionism

\begin{tabular}{lrrrrr}
\hline \hline & $\begin{array}{c}\text { Sum of } \\
\text { Squares }\end{array}$ & $d f$ & $\begin{array}{c}\text { Mean } \\
\text { Square }\end{array}$ & F & $p$ \\
\hline Sex * AS & 21.05 & 1 & 21.05 & .09 & .77 \\
Sex * DE Category & 1046.25 & 1 & 1046.25 & 4.25 & .04 \\
$\mathrm{AS} *$ DE Category & 8.60 & 1 & 8.60 & .04 & .85 \\
$\mathrm{Sex} * \mathrm{AS} *$ DE Category & 180.46 & 1 & 180.46 & .73 & .39 \\
\hline \hline
\end{tabular}


Research Question 3b: Are there any significant interactions on maladaptive or adaptive perfectionism scores between or among the independent variables of sex, athletic status, and disordered eating category?

For research question $3 \mathrm{~b}$, a 2x2x2 MANOVA was computed to determine if the hypotheses that there would be significant interactions on maladaptive perfectionism scores between or among the three independent variables; however, there would not be significant interactions on adaptive perfectionism between or among the three independent variables was supported. For this analysis, an alpha level of .025 was used in order to keep the experimentwise error rate to an acceptable level. The MANOVA revealed no significant interaction on adaptive or maladaptive perfectionism between sex and athletic status, $\mathrm{F}(2,301)=.06, p=.94$, between sex and disordered eating category, $\mathrm{F}(2,301)=1.09, p=.34$, between athletic status and disordered eating category, $\mathrm{F}(2,301)=.54, p=.58$, or among sex, athletic status, and disordered eating category, $\mathrm{F}(2,301)=.75, p=.48$ (See Table 21$)$. The results did not support the hypothesis that there would be interactions between and among the independent variables on maladaptive perfectionism; however, the results did support the hypothesis that there would not be any interactions between or among the independent variables on adaptive perfectionism. An explanation for the hypothesis regarding maladaptive perfectionism not being supported and adaptive perfectionism being supported will be given in the following chapter. 
Table 21.

Summary of Adaptive and Maladaptive Perfectionism Interactions

Summary for Adaptive and Maladaptive Perfectionism

\begin{tabular}{lcccc}
\hline \hline & $\begin{array}{c}\text { Partial Eta } \\
\text { Squared }\end{array}$ & $d f$ & F & $p$ \\
\hline Sex * AS & .00 & 2 & .06 & .94 \\
Sex * DE Category & .01 & 2 & 1.09 & .34 \\
AS * DE Category & .00 & 2 & .54 & .58 \\
Sex * AS * DE Category & .00 & 2 & .75 & .48 \\
\hline \hline
\end{tabular}

Given that there were no significant interactions found, as described in the results of research questions $3 \mathrm{a}$ and $3 \mathrm{~b}$, main effects were evaluated next. For research questions $4 \mathrm{a}, 5 \mathrm{a}$, and $6 a$, the main effects from the $2 \times 2 \times 2$ ANOVA were evaluated in order to test the hypotheses that there is a significant difference on total perfectionism scores between the comparison groups in the corresponding question. For research questions $4 b, 5 b$, and $6 b$, main effects from the $2 \times 2 \times 2$ MANOVA were evaluated to test the hypotheses that there is a significant difference on maladaptive perfectionism scores between the comparison groups in the corresponding question. It was also hypothesized that there would be no significant difference on adaptive perfectionism scores between the comparison groups in the corresponding question. For these analyses, an alpha level of .025 was used in order to keep the experimentwise error rate to an acceptable level.

Research Question 4a: Is there a significant difference on total perfectionism scores between males and females? 
As stated above, the ANOVA was evaluated to answer research question 4a. It was hypothesized that total perfectionism scores would be higher for females compared to males. Results revealed that there was not a significant difference between males and females on total perfectionism scores, $\mathrm{F}(1,302)=.23, p=.63$, which did not support the current hypothesis. See Tables 22 and 23 for a summary of the results. An explanation for this hypothesis not being supported will be given in the next chapter.

Table 22.

Descriptive Statistics for Total Perfectionism Scores in Males and Females

Total Perfectionism in Males and Females

\begin{tabular}{lccc}
\hline \hline \multicolumn{1}{c}{ Sex } & Mean & $\begin{array}{c}\text { Std. } \\
\text { Deviation }\end{array}$ & $\mathrm{N}$ \\
\hline Male & 84.55 & 16.41 & 150 \\
Female & 82.31 & 16.05 & 160 \\
& & & \\
\hline \hline
\end{tabular}

Table 23 .

Summary of Total Perfectionism Main Effect for Sex

Total Perfectionism Main Effect for Sex

\begin{tabular}{lrcccc}
\hline \hline & $\begin{array}{c}\text { Sum of } \\
\text { Squares }\end{array}$ & $d f$ & $\begin{array}{c}\text { Mean } \\
\text { Square }\end{array}$ & F & $p$ \\
\hline Sex & 57.58 & 1 & 57.58 & .23 & .63 \\
\hline \hline
\end{tabular}

Research Question 4b: Is there a significant difference on maladaptive or adaptive perfectionism scores between males and females?

The MANOVA tested the hypotheses that females would have higher maladaptive perfectionism scores than males; however there would not be a significant difference on adaptive 
perfectionism scores between males and females. The multivariate analysis looking at the linear combination of adaptive and maladaptive perfectionism was not significant on the variable of sex, $\mathrm{F}(2,301)=.90, p=.41$, which is not in support of the hypotheses that there would be differences on maladaptive perfectionism scores, but is in support of the hypothesis that there would not be differences on adaptive perfectionism scores (See Tables 24 and 25). Therefore, it was inappropriate to evaluate the between-subject results for these two dependent variables on the independent variable of sex. A discussion about the partial support of this hypothesis will be offered in the following chapter.

Table 24.

Descriptive Statistics for Maladaptive and Adaptive Perfectionism Scores in Males and Females Maladaptive and Adaptive Perfectionism in Males and Females

\begin{tabular}{llccc}
\hline \hline & Sex & Mean & $\begin{array}{c}\text { Std. } \\
\text { Deviation }\end{array}$ & $\mathrm{N}$ \\
\hline Maladaptive & Male & 58.89 & 13.41 & 150 \\
& Female & 57.38 & 13.49 & 160 \\
Adaptive & Male & 47.87 & 7.19 & 150 \\
& Female & 48.24 & 7.62 & 160 \\
\hline \hline
\end{tabular}

Table 25.

Summary of Maladaptive and Adaptive Perfectionism Main Effect for Sex Maladaptive and Adaptive Perfectionism Main Effect for Sex

\begin{tabular}{lcccc}
\hline \hline & $\begin{array}{c}\text { Partial Eta } \\
\text { Squared }\end{array}$ & $d f$ & F & $p$ \\
\hline Sex & .01 & 2 & .90 & .41 \\
\hline \hline
\end{tabular}


Research Question 5a: Is there a significant difference on total perfectionism scores between athletes and non-athletes?

The ANOVA was evaluated to answer research question 5a. It was hypothesized that athletes would have higher scores on total perfectionism compared to non-athletes. There was not a significant difference between athletes and non-athletes on total perfectionism scores, $\mathrm{F}(1,302)=.03, p=.86$, which again does not support the hypothesis of the current study (See Tables 26 and 27). A discussion of this hypothesis not being supported will be given in the next chapter.

Table 26.

Descriptive Statistics for Total Perfectionism Scores in Athletes and Non-Athletes

Total Perfectionism in Athletes and Non-Athletes

\begin{tabular}{lccc}
\hline \hline \multicolumn{1}{c}{ AS } & Mean & $\begin{array}{c}\text { Std. } \\
\text { Deviation }\end{array}$ & $\mathrm{N}$ \\
\hline Athletes & 83.61 & 14.18 & 119 \\
Non-Athletes & 83.26 & 17.43 & 191 \\
\hline \hline
\end{tabular}

Table 27.

Summary of Total Perfectionism Main Effect for Athletic Status

Total Perfectionism Main Effect for Athletic Status

\begin{tabular}{lrrrrr}
\hline \hline & $\begin{array}{c}\text { Sum of } \\
\text { Squares }\end{array}$ & $d f$ & $\begin{array}{c}\text { Mean } \\
\text { Square }\end{array}$ & F & $p$ \\
\hline Athletic Status & 7.29 & 1 & 7.29 & .03 & .86 \\
& & & & & \\
\hline \hline
\end{tabular}


Research Question 5b: is there a significant difference on maladaptive or adaptive perfectionism between athletes and non-athletes?

The MANOVA tested the hypothesis that athletes have higher maladaptive perfectionism scores compared to non-athletes; however, there is not a difference between athletes and nonathletes on adaptive perfectionism scores. The multivariate analysis looking at the linear combination of adaptive and maladaptive perfectionism was not significant on the variable of athletic status, $\mathrm{F}(2,301)=1.85, p=.16$, again not supporting the hypotheses regarding maladaptive perfectionism scores, but supporting the hypothesis regarding adaptive perfectionism scores (See Tables 28 and 29). Therefore, it was inappropriate to evaluate the between-subject results for these two dependent variables on the independent variable of athletic status. An explanation of this hypothesis being partially supported and partially not supported will be offered in the following chapter.

Table 28.

Descriptive Statistics for Maladaptive and Adaptive Perfectionism Scores in Athletes and NonAthletes

Maladaptive and Adaptive Perfectionism in Athletes and Non-Athletes

\begin{tabular}{llccc}
\hline \hline & \multicolumn{1}{c}{ AS } & Mean & $\begin{array}{c}\text { Std. } \\
\text { Deviation }\end{array}$ & $\mathrm{N}$ \\
\hline Maladaptive & Athlete & 57.13 & 12.08 & 119 \\
& Non-Athlete & 58.72 & 14.24 & 191 \\
\multirow{2}{*}{ Adaptive } & Athlete & 48.57 & 6.83 & 119 \\
& Non-Athlete & 47.74 & 7.74 & 191 \\
\hline \hline
\end{tabular}


Table 29.

Summary of Maladaptive and Adaptive Perfectionism Main Effect for Athletic Status

Maladaptive and Adaptive Perfectionism Main Effect for AS

\begin{tabular}{lcccc}
\hline \hline & $\begin{array}{c}\text { Partial Eta } \\
\text { Squared }\end{array}$ & $d f$ & $\mathrm{~F}$ & $p$ \\
\hline Athletic Status & .01 & 2 & 1.85 & .16 \\
\hline \hline
\end{tabular}

Research Question 6a: Is there a significant difference on total perfectionism scores between the asymptomatic and symptomatic categories of disordered eating?

The ANOVA was evaluated to determine if the symptomatic category of disordered eating had higher scores on total perfectionism compared to the asymptomatic category of disordered eating. There was a significant difference found between the symptomatic and asymptomatic categories of disordered eating on total perfectionism scores, $\mathrm{F}(1,302)=16.61, p$ $=<.01$. The symptomatic category had significantly higher total perfectionism scores than the asymptomatic category, which supported the current hypothesis. Tables 30 and 31 provide a summary of this analysis. The following chapter will contain a discussion about the support of this hypothesis. 
Table 30.

Descriptive Statistics for Total Perfectionism Scores in Asymptomatic DE Category and Symptomatic DE Category

Total Perfectionism in Asymptomatic and Symptomatic

\begin{tabular}{cccc}
\hline \hline DE Category & Mean & $\begin{array}{c}\text { Std. } \\
\text { Deviation }\end{array}$ & $\mathrm{N}$ \\
\hline Asymtpomatic & 80.96 & 14.38 & 223 \\
Symptomatic & 89.64 & 18.94 & 87 \\
\hline \hline
\end{tabular}

Table 31.

Summary of Total Perfectionism Main Effect for Disordered Eating Category

Total Perfectionism Main Effect for Disordered Eating Category

\begin{tabular}{llllll}
\hline \hline & $\begin{array}{c}\text { Sum of } \\
\text { Squares }\end{array}$ & $d f$ & $\begin{array}{c}\text { Mean } \\
\text { Square }\end{array}$ & F & $p$ \\
\hline Disordered Eating Category & 4087.61 & 1 & 4087.61 & 16.61 & $<.01$ \\
& & & & & \\
\hline \hline
\end{tabular}

Research Question 6b: Is there a significant difference on maladaptive or adaptive perfectionism scores between the asymptomatic and symptomatic categories of disordered eating?

The MANOVA tested the hypothesis that the symptomatic category of disordered eating would have higher maladaptive perfectionism scores than the asymptomatic category of disordered eating; however, there would not be significant differences between the two disordered eating categories on adaptive perfectionism scores. The multivariate analysis looking at the linear combination of adaptive and maladaptive perfectionism was significant on the variable of disordered eating category, $\mathrm{F}(2,301)=12.76, p=<.01$, which supports the hypothesis 
(See Tables 32 and 33). Given the significant difference found on the multivariate analysis, it was appropriate to evaluate the between-subject results for these two dependent variables on the independent variable of disordered eating category to determine if the significant difference between the two categories was on maladaptive perfectionism scores, adaptive perfectionism scores, or both. A discussion about this hypothesis being supported will be given in the following chapter. Results of the ANOVA revealed there was a significant difference between the symptomatic and asymptomatic categories on maladaptive perfectionism scores, $\mathrm{F}(1,302)=$ 23.61, $p=<.01$, with the symptomatic category having higher scores on maladaptive perfectionism than the asymptomatic category, which supported the hypothesis for this research question. A discussion of these results will be presented in the following chapter.

Table 32.

Descriptive Statistics for Maladaptive and Adaptive Perfectionism Scores in Asymptomatic and Symptomatic DE Categories

Maladaptive and Adaptive Perfectionism in Asymptomatic and Symptomatic DE Categories

\begin{tabular}{lcccc}
\hline \hline & DE Category & Mean & $\begin{array}{c}\text { Std. } \\
\text { Deviation }\end{array}$ & N \\
\hline Maladaptive & Asymptomatic & 55.77 & 12.09 & 223 \\
& Symptomatic & 64.10 & 14.91 & 87 \\
Adaptive & Asymptomatic & 48.00 & 7.31 & 223 \\
& Symptomatic & 48.21 & 7.68 & 87 \\
\hline \hline
\end{tabular}


Table 33 .

Summary of Maladaptive and Adaptive Perfectionism Main Effect for DE Category

Maladaptive and Adaptive Perfectionism Main Effect for DE Category

\begin{tabular}{lcccc}
\hline \hline & $\begin{array}{c}\text { Partial Eta } \\
\text { Squared }\end{array}$ & $d f$ & $\mathrm{~F}$ & $p$ \\
\hline Disordered Eating Category & .08 & 2 & 12.76 & $<.01$ \\
\hline \hline
\end{tabular}

\section{Summary of the Results}

In summary, results indicated that total and maladaptive perfectionism scores significantly varied based on the category of disordered eating (asymptomatic, symptomatic, eating disordered), while adaptive perfectionism did not vary based on the category of disordered eating. This supported hypotheses 1a, 1b, and 1c of the current study. Hypothesis $2 \mathrm{a}$ was supported also, as results revealed that there was a significant difference in the frequencies between males' and females' in the disordered eating categories. However, hypotheses $2 \mathrm{~b}$ and $2 \mathrm{c}$ were not supported as there was no significant difference in the frequencies between athletes' and non-athletes' in the disordered eating categories nor was there a significant difference in the frequencies between male athletes', female athletes', male non-athletes', and female nonathletes' in the disordered eating categories. There were also no significant interactions found between or among sex, athletic status, and disordered eating category for either total perfectionism scores or maladaptive or adaptive perfectionism scores, which did not support hypotheses $3 \mathrm{a}$ and partially supported hypothesis $3 \mathrm{~b}$ with regards to there being no significant interactions between or among the variables on adaptive perfectionism. Males and females did not differ significantly on their total perfectionism scores, failing to support hypothesis $4 \mathrm{a}$. Hypothesis $4 \mathrm{~b}$ was partially supported as males and females did not differ on adaptive 
perfectionism scores as predicted; however, they also did not differ on maladaptive perfectionism scores, which did not support the hypothesis. Athletes and non-athletes also did not differ significantly on their total perfectionism scores, which did not support hypothesis 5a. However, similar to hypothesis $4 \mathrm{~b}$, hypothesis $5 \mathrm{~b}$ was partially supported due to their not being significant differences on adaptive perfectionism scores between athletes and non-athletes as predicted; however, athletes and non-athletes also did not differ significantly on their maladaptive perfectionism scores, going against the current hypothesis. Finally, hypotheses $6 \mathrm{a}$ and $6 \mathrm{~b}$ were fully supported, as the symptomatic and asymptomatic categories of disordered eating had significantly different scores on both total perfectionism and maladaptive perfectionism, but did not have significantly different scores on adaptive perfectionism. The implication of these results will be discussed in the next chapter. 


\section{Chapter 5 - Discussion}

This chapter will address the implications and explanations of the findings presented in Chapter 4. First, the findings from the statistical analyses will be discussed with possible explanations given for the findings. This will include a discussion of the explanation for both the findings that supported the hypotheses and agreed with the findings from the previous literature reviewed earlier and the findings that did not support the hypotheses and conflicted with the literature reviewed earlier. Next, strengths and limitations of the current study will be reviewed. Finally, recommendations and suggestions for future research and clinical applications will be discussed.

\section{Findings}

The purpose of the study was to determine the amounts of disordered eating (asymptomatic, symptomatic, and eating disordered) and perfectionism (total, adaptive, and maladaptive) in college students. Specifically, the study was designed to establish if scores on the three types of perfectionism varied across the disordered eating categories; if males and females, athletes and non-athletes, and male athletes, female athletes, male non-athletes, and female non-athletes differed in their frequencies in the disordered eating categories (asymptomatic, symptomatic); and if there were significant differences on perfectionism scores (total, maladaptive, adaptive) between males and females, athletes and non-athletes, and the asymptomatic and symptomatic categories of disordered eating. The findings of this study revealed that there is a relationship between disordered eating and perfectionism, that males and females differed in their frequencies in the disordered eating categories, and that total and maladaptive perfectionism scores were significantly different for the asymptomatic and symptomatic categories of disordered eating. However, the findings did not indicate that there 
were differences between athletes and non-athletes in frequencies in disordered eating categories or any differences on either total or maladaptive perfectionism between males and females or athletes and non-athletes.

Hypotheses 1a predicted that total perfectionism would vary based on category of disordered eating, with the asymptomatic category having the lowest mean total perfectionism score, followed by the symptomatic category, and finally the eating disordered category having the highest mean total perfectionism scores. This hypothesis was supported, as total perfectionism group means varied based on disordered eating category. The asymptomatic category had the lowest mean score, while the eating disordered category had the highest mean score. The asymptomatic category was significantly different than both the symptomatic and eating disordered categories. The findings for hypothesis $1 \mathrm{~b}$ were similar, only regarding maladaptive perfectionism scores. This hypothesis also was supported, as maladaptive perfectionism group means varied based on disordered eating category, and again the asymptomatic category had the lowest mean maladaptive perfectionism score while the eating disorder category had the highest mean maladaptive perfectionism score. Again, the asymptomatic category was significantly different than both the symptomatic and eating disordered categories.

These findings are important to the field of psychology, as they provide evidence for using a continuum model of disordered eating, as the results revealed that those with a diagnosable eating disorder, those with symptoms of an eating disorder, and those with no symptoms of an eating disorder differ on amounts of perfectionism. Typically in research studies participants are classified as either having a diagnosable eating disorder or not having a diagnosable eating disorder. The findings of this study demonstrate the importance of separating 
those who have no symptoms of a diagnosable eating disorder and those who are displaying symptoms of a diagnosable eating disorder, as they may vary on important factors related to disordered eating, including perfectionism. These findings agree to an extent with those of Tylka and Subich (1999) who found that high school and college aged females' scores on a unidimensional measure of perfectionism did not vary based on disordered eating category and Tylka and Subich (2002) who found that high school and college aged males' scores on a unidimensional measure of perfectionism did not vary based on disordered eating category. Hypothesis 1c stated that adaptive perfectionism mean scores would not vary based on disordered eating category, and this hypothesis was also supported. The finding that adaptive perfectionism did not vary based on disordered eating category is especially important and lends an enormous amount of support for using a multidimensional model of perfectionism. These findings have shown that perfectionism can be both adaptive or positive and maladaptive or negative, and that it is the maladaptive or negative aspects of perfectionism that are associated with disordered eating behaviors rather than the adaptive or positive aspects of perfectionism. Hamachek (1978) referred to these two types of perfectionism as normal (adaptive) perfectionism and neurotic (maladaptive) perfectionism. Hamachek described normal perfectionists as setting high standards, receiving pleasure from their efforts, and those "who feel free to be less precise as the situation permits" (p. 27). Normal perfectionists are also able to consider both their strengths and weaknesses and set realistic expectations for themselves (Hamachek, 1978). On the other hand, neurotic perfectionists are seen as 'never good enough' in their own eyes, no matter how successful their efforts (Hamachek, 1978). Hamachek stated that they typically "demand of themselves a higher level of performance than is usually possible to attain" (p. 27). The other big difference between normal and neurotic perfectionists that 
Hamachek noted was that normal perfectionists are motivated by "a desire for improvement" whereas neurotic perfectionists are motivated by "fear of failure" (p. 28). Given this, those with higher levels of disordered eating behavior would tend to see themselves as not good enough, set unrealistic goals, and be motivated by failure more so than those with lower amounts of disordered eating behaviors. This may explain the perpetuation of disordered eating behaviors, as they feel that they are never achieving their end goal, which more than likely is an unrealistic goal such as an unattainable weight.

Due to the small number of participants who were classified as eating disorder $(\mathrm{N}=12)$, the remaining analyses were only completed on participants classified as asymptomatic or symptomatic. Even though this removes one of the three categories of disordered eating, it still allows for support of the continuum model as it is comparing those who have symptoms of an eating disorder (symptomatic) to those who have no symptoms of an eating disorder (asymptomatic).

Hypothesis 2a predicted that a difference would exist between males' and females' frequencies in the disordered eating categories (asymptomatic, symptomatic) with more males in the asymptomatic category and more females in the symptomatic category. This hypothesis was supported, as there were males than expected in the asymptomatic category and more females than expected in the symptomatic category. This finding contradicts several of the studies reviewed earlier that found males and females were similar in their disordered eating behaviors (Crisp \& Burns, 1990; Edwin \& Andersen, 1990; Hay et al., 2005; Woodside et al., 1990). However, all of those studies had participants who were diagnosed with an eating disorder and used a discontinuum measure of disordered eating. This finding does agree with the findings of Furnham et al. (2002) and Varnado-Sullivan (2006) who found that males and females differed 
in their degrees of disordered eating behaviors, with females engaging in more disordered eating behaviors, although again discontinuum measures of disordered eating were utilized in both studies.

There was not a significant difference found in the frequencies between athletes and nonathletes in the disordered eating categories, which went against hypothesis $2 \mathrm{~b}$. There was also not a significant difference found in the frequencies between male athletes, female athletes, male non-athletes, and female non athletes in the disordered eating categories, which did not support hypothesis $2 c$. These findings contradict the findings of several studies reviewed earlier in this document that all found athletes to have higher levels of disordered eating behaviors than nonathletes (Byrne \& McLean, 2001; Hausenblas \& Carron, 1999; Johnson et al., 1999; Petrie \& Rogers, 2001; Pritchard et al., 2007; Smolak et al., 2000; Sundgot-Borgen \& Torstveit, 2004; Szymanski \& Chrisler, 1990-1991). Specifically, Byrne and McLean (2001), Hausenblas and Carron (1999), Pritchard et al. (2007), and Sundgot-Borgen and Torstveit (2004) all found that female athletes had more symptoms of disordered eating than female non-athletes. There were also several researchers who found that male athletes tended to have higher levels of disordered eating symptoms compared to male non-athletes (Byrne \& McLean, 2001; Husenblas \& Carron, 1999; Pritchard et al., 2007; Sundgot-Borgen \& Torstveit, 2004). However, these results do agree with the findings of Fulkerson et al. (1999) who found that neither female athletes or male athletes differed from female non-athletes and male athletes, respectively, on several measures of disordered eating. Finally, several researchers had also found that female athletes engaged in more disordered eating behaviors than male athletes (Byrne \& McLean, 2001; Johnson et al., 1999; Sundgot-Borgen \& Torstveit, 2004), while Hausenblas and Carron (1999) found that male athletes engaged in more disordered eating behaviors than female athletes. 
There are several possible explanations for the results not supporting hypotheses $2 \mathrm{~b}$ and 2c. The first possible explanation revolves around the types of sports that were surveyed as part of this study. Male athlete participants were taken primarily from the baseball and cross country teams, while female athlete participants were taken primarily from the volleyball, crew/rowing, soccer, and cross country teams. With the exception of the cross country teams and possibly volleyball, these are not sports that are typically associated with engaging in disordered eating behaviors in order to maintain a body type for their specific sport. Another possible explanation revolves around the fact that the athletes all completed the questionnaires in a room with the other members of their team. Some of these participants could have felt compelled to answer in a specific way, therefore leading to a socially desirable response set, for fear of their coach finding out about possible disordered eating behaviors and therefore receiving consequences for these behaviors. Another explanation is that a majority of the participants were younger (i.e., freshman and sophomores), and while the athlete participants are taking part in weight maintenance and reduction activities as part of their sport (i.e., practice activities such as running, weight lifting, etc.), non-athlete participants may either be experiencing or fear experiencing the common "freshman 15" that often occurs in college. Due to this fear or experience, they may be participating in disordered eating behaviors to combat this weight gain, therefore, making nonathletes experience a similar amount of disordered eating symptoms at the athletes. A final explanation is that similar to what Fulkerson et al. found, there may actually be no difference in frequencies in the disordered eating categories between athletes and non athletes or between male athletes, female athletes, male non-athletes, and female non-athletes.

Hypotheses 3 a stated that there would be interactions on total perfectionism scores between and among sex, athletic status, and disordered eating category. This hypothesis was not 
supported as there were no significant interactions on total perfectionism scores between or among any of the independent variables. Hypothesis $3 \mathrm{~b}$ predicted that there would be interactions on maladaptive perfectionism scores between and among sex, athletic status, and disordered eating category; however, there would not be significant interactions on adaptive perfectionism between or among sex, athletic status, and disordered eating categories. The part of the hypothesis dealing with maladaptive perfectionism was not supported as there were no significant interactions between or among those three variables; however, the finding that there were no interactions on adaptive perfectionism between or among the variables lends partial support to the hypothesis. This finding indicates that the effect of one IV is not dependent on the level of another IV, or in other words, the effect of sex, athletic status, or disordered eating category is not dependent on the level of any other one for total, maladaptive, or adaptive perfectionism.

One explanation for hypotheses $3 \mathrm{a}$ and $3 \mathrm{~b}$ not being supported is the type of student found at the university where the study took place. This particular university has very high standards for students, with the average high school GPA of incoming freshman being 3.76 and the average ACT score being 26.58. Therefore, these high standards may lead all students, athlete or non-athlete, male or female, asymptomatic or symptomatic disordered eating category, to having high levels of perfectionism. This university takes to heart that those attending the university and participating on an athletic team are "student-athletes" in that order. A second explanation of the current findings not supporting the hypotheses is that there truly are no interactions on any of the types of perfectionism between or among sex, athletic status, and disordered eating category. 
Against hypothesis 4a, it was found that males and females did not differ significantly on mean total perfectionism scores. It was also found that males and females did not differ significantly on maladaptive or adaptive mean perfectionism scores. This partially supports hypothesis $4 \mathrm{~b}$ which predicted that mean maladaptive perfectionism scores would be higher for females, but adaptive perfectionism scores would be similar for males and females. The results of the current study concur with those of Forbush et al. (2007) who also found no difference in levels of perfectionism between males and females; however, Forbush et al. used a unidimensional model of perfectionism. Slane et al. (2010) also found no difference in levels of Concern Over Mistakes (CM), a subscale on the Frost Multidimensional Perfectionism Scale (Frost MPS) that is used in the calculation of both total and maladaptive perfectionism. Macedo et al. (2007), however, found a significant difference in maladaptive perfectionism between males and females, with males having higher levels of maladaptive perfectionism than females, which is the opposite of what was hypothesized in the current study. Woodside et al. (2004) found that females were more perfectionistic than males; however, they only used participants who were diagnosed with an eating disorder, which is not congruent with the sample from the current study.

Again, there are two possible explanations for this finding. First, as stated above, the type of student at the institution where the study was completed could have impacted these results, as all students may be engaging in maladaptive perfectionistic behaviors in order to meet the high academic standards of the university that are expected of them. The second possible explanation is that there really is no difference in the levels of total and maladaptive perfectionism between males and females. 
Hypothesis 5a stated that athletes would have a significantly higher total perfectionism mean compared to non-athletes, which was not supported by the analysis. Hypothesis $5 \mathrm{~b}$ stated that athletes would have a significantly higher maladaptive perfectionism mean than non-athletes and there would be no difference on adaptive perfectionism means between athletes and nonathletes was partially supported. That is, there were no significant differences on adaptive perfectionism means between the two groups. When examining differences in perfectionism levels among athletes, Symanski and Chrisler (1990-1991) found that athletes had higher levels of perfectionism than non-athletes, which contradicts the findings of the current study, although they used a unidimensional measure of perfectionism. Fulkerson et al. (1999), who also used a unidimensional measure of perfectionism, found no differences in levels of perfectionism between female athletes and female non-athletes or between male athletes and non-athletes which is consistent with the current findings.

One possible explanation for no significant differences found on mean total and maladaptive perfectionism scores between athletes and non-athletes is that there may be more focus on academics at this university than there is on athletics. Coaches may push their athletes to perform well in the classroom, and make that a more important goal than their performance in the athletic arena. Most of the members of college teams at this university are required to participate in study tables, even if their GPAs are at an acceptable point. This demonstrates the commitment to academics by the university as a whole, including the athletic department. Related to the first explanation, students may already be engaging in maladaptive perfectionistic behaviors and thoughts as part of their "student role" and therefore amounts of maladaptive and total perfectionism may already be high due to this role without taking into consideration their athletic status. 
Finally, hypothesis $6 a$ was supported in that the symptomatic category had a significantly higher mean total perfectionism score than the asymptomatic category. Hypothesis $6 \mathrm{~b}$ was also supported as the symptomatic category also had a significantly higher mean maladaptive perfectionism score than the asymptomatic category, and there was not a significant difference on mean adaptive perfectionism scores between the two categories.

These results lend evidence to maladaptive or negative perfectionism being related to disordered eating, but adaptive or positive perfectionism not being related to disordered eating. Similar to the results for hypotheses $1 \mathrm{a}, 1 \mathrm{~b}$, and $1 \mathrm{c}$, these results lend support to the continuum model of disordered eating and the multidimensional model of perfectionism. As was stated earlier, most studies typically compare individuals with a diagnosable eating disorder and individuals without a diagnosable eating disorder. These results demonstrate the importance of separating out those not diagnosed with an eating disorder into two groups: those who display symptoms of an eating disorder and those who do not display symptoms of an eating disorder, as the two groups may differ on some very important facets including perfectionism. These results also demonstrate the importance of viewing perfectionism as a multidimensional construct, as not all types of perfectionism are associated with disordered eating behaviors.

\section{Strengths and Limitations}

The main strength of the current study is that this is the first study to use both a continuum model of disordered eating and a multidimensional model of perfectionism. The results of research questions $1 \mathrm{a}, 1 \mathrm{~b}$, and $1 \mathrm{c}$ all support the use of the continuum model of disordered eating, as it was found that amounts of total and maladaptive perfectionism varied across disordered eating categories, demonstrating that individuals with varying amounts of disordered eating behaviors differ on important characteristics such as total and maladaptive 
perfectionism. The results of research questions $6 \mathrm{a}$ and $6 \mathrm{~b}$ also support the use of a continuum model of disordered eating, as both total perfectionism mean scores and maladaptive perfectionism mean scores were significantly different between the asymptomatic category and the symptomatic category. There were also differences found in the frequency of males and females in the asymptomatic and symptomatic categories, demonstrating support for the continuum model. Even though the frequency of athletes and non-athletes in the two categories did not differ significantly, there were still some participants from each of those groups in both categories, demonstrating that eating disorders are not just an 'either or' type of variable.

Much of the past research has divided participants into those who have a diagnosable eating disorder and those who do not have a diagnosable eating disorder, therefore putting those individuals who may have some disordered eating in the same category as those with no symptoms of an eating disorder, when in actuality these two populations may vary on important facets including their degree of total and maladaptive perfectionism as demonstrated by this study. The idea of a continuum model of eating disorders, where people are classified as either having a diagnosable eating disorder, having symptoms of an eating disorder, or being symptom free is supported by several researchers (Cohen \& Petrie, 2005; Mintz \& Betz, 1988; Tylka \& Subich, 1999; Tylka \& Subich, 2002). Sundgot-Borgen and Torstveit (2004) found that more people, both male and female, met criteria for subclinical eating disorders than for clinical eating disorders, which would also lend support to viewing eating disorders on a continuum. Hausenblas and Carron (1999) actually encourage researchers to use the Q-EDD, as was used in the current study, due to it measuring differences between those who have no symptoms of an eating disorder (asymptomatic), some symptoms of an eating disorder (symptomatic), and a diagnosable eating disorder (eating disordered). 
The current study also lends support to the use of a multidimensional model of perfectionism. The results for research questions 1a, 1b, and 1c again revealed that scores on total and maladaptive perfectionism varied across the disordered eating categories; however, scores on adaptive perfectionism did not vary across the disordered eating categories. Also, the results of research questions $6 \mathrm{a}$ and $6 \mathrm{~b}$, that there were significant differences on total and maladaptive perfectionism scores between the asymptomatic and symptomatic categories; however, there was not a significant difference on adaptive perfectionism scores between these two categories, also lends support to the use of a multidimensional perspective of perfectionism. This shows that not all types of perfectionistic attitudes and behaviors are the same and that some of them, such as maladaptive or negative forms of perfectionism, are more related to disordered eating behaviors than other types, such as adaptive or positive forms of perfectionism. The distinction between adaptive and maladaptive perfectionism centers around the types of expectations people set for themselves, what motivates people, and the feelings associated with efforts put forth by the person (Hamachek, 1978). Adaptive perfectionism is associated with setting realistic expectations, being motivated by "a desire for improvement," and receiving pleasure from efforts (Hamachek, 1978, p. 28). Conversely, maladaptive perfectionism is connected to setting unrealistic expectations, being motivated by "fear of failure," and feelings of never being good enough (Hamachek, 1978, p. 28).

Some may view the removal of the eating disordered category participants as a limitation as it removed one of the three categories of disordered eating from a majority of the analyses. Due to the small number of participants who were classified as eating disordered on the Q-EDD $(\mathrm{N}=12$ ), analyses could not be completed on this group with strong confidence. To a small degree, this limits the continuum model of disordered eating; however, it does not completely 
eliminate this model from the study. As stated above, a majority of previous research has compared those with a diagnosable eating disorder to those without a diagnosable eating disorder, combining those who have symptoms of an eating disorder but do not meet diagnostic criteria and those who have no symptoms of an eating disorder in one group. The current study was able to differentiate between those who display symptoms of an eating disorder and those who do not display symptoms of an eating disorder, which still upholds the continuum model and also lends evidence to these two groups being different on variables such as perfectionism. Although it is a slight limitation that the eating disorder category could not be included in the majority of analyses, it is also a strength, as the study demonstrated that those who exhibit symptoms of an eating disorder but do not qualify for a diagnosis and those who do not exhibit symptoms of an eating disorder should not be viewed as one in the same.

As with any study, there are limitations to the current study. The biggest limitation to the current study is the homogeneity of the sample. As is evidenced from the age, class status, and race/ethnicity percentages presented in the previous chapter, the sample lacked diversity in all of these areas. This was especially prevalent in the race/ethnicity category, as over $81 \%$ of all participants identified as Caucasian/White. The race/ethnicity percentages within just the athlete participants was even less diverse, with over $92 \%$ of athlete participants identifying as Caucasian/White. The homogeneity of the current sample is similar to the homogeneity of the campus population where the study was conducted. At this university, $74.2 \%$ of all students (undergraduate and graduate) identify as Caucasian and $21.3 \%$ are classified as minority for their ethnic composition. Other ethnic identifications for the university include $9 \%$ Asian, 5\% Hispanic, 3.3\% African American, 3.1\% have two or more races, 0.5\% Native American, 0.4\% 
Native Hawaiian/Pacific Islander, $2 \%$ are international students, and 2.5\% listed unknown as their ethnic composition.

A related limitation is the restricted access to a variety of students as the study used a convenience cluster sampling procedure. All of the non-athlete participants were recruited through either the Psychology Department or the Student Life Division of the university and the athlete participants were decided by which coaches responded to the invitation email, both of which restrict access to a variety of students. Given this sampling method, there is a chance that the sample is not completely representative of the students on campus, as not every single student had an equal chance of being chosen to participate in the study. In addition, there was not access to sports that are more likely to engage in disordered eating behaviors, such as football, wrestling, and gymnastics, as these sports are not offered at the university where the study was completed. There may have been greater variability across the disordered eating categories if access to specific types of athletes or sports would have been available.

Issues surrounding external validity, or the extent to which the results are generalizable, is another limitation to the current study. The average participant was a 19-year-old, White college student at a prestigious university in the Midwest. This could lead to some selection effects of the sample (Cozby \& Bates, 2012). Using college students will help us understand this population in general, but will not be generalizable to other populations such as other ages (e.g., high school students, middle aged adults, or older adults) or athletic levels (e.g., high school athletes or elite athletes). Another generalizability issue lies in the fact that the sample is coming from a large, prestigious, Division I, Midwestern university, which may not be generalizable to other universities in the United States or outside of the United States. Also, the variables of sex and athletic status were pre-determined as they were classifications that already existed either 
based on their biological sex or their membership on an NCAA team at the selected university. The attitudes and behaviors of the sample may also not be reflective of the attitudes and behaviors of other college students or the general population.

There is also the possibility that demand characteristics, "cues that inform the subject how he or she is expected to behave" influenced the study (Cozby \& Bates, 2012, p. 185). Some non-athlete participants signed up for the study using a sign-up sheet, which solicited participants for a study about perfectionism and eating behaviors. Other non-athlete participants were solicited by the researcher telling the class that they were being asked to participate in a study about perfectionism and eating behaviors. And finally, athletes were solicited through emails to coaches which stated that the athletes would be asked questions about perfectionism and eating behaviors and then the athletes themselves were told that the study would ask these types of questions. Using these words in the solicitation of participants could have influenced the types of people that decided to participate in the study. Perhaps, people who had more disordered eating behaviors or high levels of perfectionism chose not to participate so they did not have to reveal these behaviors. No participant was told the hypotheses of the study prior to participating; however, some participants could have guessed correctly at the study hypotheses and based their answers to the questions on this guess. Participants could have also responded in a biased way, creating a socially desirable response set, even though they were ensured of confidentiality. More specifically, the participants could have answer the questions in a manner to minimize their disordered eating behaviors or perfectionistic tendencies in fear of receiving negative consequences from an authority figure such as a professor or coach.

A final limitation to the study is that participants self-reported their height and weight, which were used to calculate BMIs. Each participant's BMI is used in the process of placing 
them into one of the three categories of disordered eating. An obvious issue with this is the possibility of participants not being honest when reporting their height and weight, which would influence the calculation of their BMI, therefore influencing the participant's placement into the appropriate disordered eating category. Also, while scoring the Q-EDD, the researcher noted that most of the self-reported weights ended in a 0 or 5 . For example, people would self-report weight more often as 120 pounds or 175 pounds compared to 122 pounds or 178 pounds. Even though these self-reported weights may have only varied from true weights by a few pounds, this still could have influenced their BMI calculations, which again would influence their placement into the proper disordered eating category.

\section{Recommendations and Suggestions for Future Research and Clinical Applications}

The results of this study confirmed the hypotheses that total and maladaptive perfectionism scores varied across disordered eating category, while adaptive perfectionism scores did not vary across disordered eating category. The results also confirmed the hypothesis that males and females differed in their frequencies in the disordered eating categories. Finally, the results supported the hypotheses that total and maladaptive perfectionism scores differed between the symptomatic and asymptomatic categories of disordered eating, while adaptive perfectionism scores did not differ between the symptomatic and asymptomatic categories. This is mostly in support of the literature reviewed in an earlier chapter that there is a relationship between perfectionism and disordered eating behaviors. The results did not support the hypotheses that athletes and non-athletes and male athletes, female athletes, male non-athletes, and female non-athletes would differ in their frequencies in the disordered eating categories. The results also did not confirm the hypotheses that there would be interactions on total or maladaptive perfectionism between and among sex, athletic status, and disordered eating 
category, although the prediction that there would not be interactions on adaptive perfectionism between or among these variables was confirmed. Finally, the results did not support the hypotheses that there would be significant differences on total and maladaptive perfectionism scores between males and females or between athletes and non-athletes; however, the prediction that adaptive perfectionism scores would not differ between males and females or athletes and non-athletes was supported. These finding concur with some of the literature reviewed earlier, but disagrees with other literature reviewed earlier.

As is stated in the strengths part of this discussion, this was the first study to assess scores on perfectionism and disordered eating behaviors using both a multidimensional perspective of perfectionism and a continuum model of disordered eating. The results of this study lend evidence to using both a continuum model of disordered eating and a multidimensional model of perfectionism. Given this, it is imperative that additional research be completed on this topic using a multidimensional perspective of perfectionism and a continuum model of disordered eating in order to increase our knowledge and understanding and lend further evidence to the use of these models. Given the above discussed results and the need for additional research using a multidimensional perspective of perfectionism and continuum model of disordered eating, the following suggestions are offered to advance research in this field.

Participants. Future research in this area could use a more diverse sample of participants. As was stated earlier, the average participant was a 19-year old, White college student from a prestigious, Division I Midwestern university. As discussed above, this may have influenced the results in several ways. Future research could obtain samples that are more ethnically and racially diverse in order to increase knowledge about possible racial and ethnic differences in disordered eating behaviors and perfectionistic attitudes. If using college students, 
future research could use a sampling method that would either ensure random sampling from the population or a sampling method that would ensure a more even distribution of ages, class statuses, and races/ethnicities. Given that pure random sampling is rare, attaining a more equal distribution of ages, class statuses, and races/ethnicities may be more realistic. By obtaining a more equal distribution of ages, class statuses, and races/ethnicities, the results may be more generalizable to college populations.

Future research could also broaden the research by using a different university, or possibly comparing several colleges or universities. Given the current sample was from a rather prestigious university in the Midwest, the results may not be generalizable to colleges in different areas or that do not have similar academic standards. If future researchers were to use other schools that were more similar to typical colleges in the United States, the results may more accurately reflect the college student population in the United States. By comparing several different colleges or universities, researchers could find differences between small sized colleges (i.e. Division III), medium sized colleges/universities (i.e., Division II), and large sized universities (i.e., Division I) to see if scores on the different types of perfectionism and disordered eating behaviors varied based on school size and level of athletic competition. The research could also be broadened by studying and/or comparing specific sports to see if scores on the three types of perfectionism and disordered eating behaviors vary based on the sport. For example, research could look at whether these two variables differ between athletes participating in lean sports such as track/cross country and swimming and those participating in non-lean sports such basketball and soccer.

Research could also be broadened by using elite athletes or high school athletes rather than college athletes. By using elite athletes, it would increase understanding of the 
perfectionistic attitudes and disordered eating behaviors that may be experienced due to this higher level of competition. By using high school athletes, several different sizes of high schools could be compared, similar to the suggestion about colleges/universities, in order to see if there are differences in the scores on the three types of perfectionism and differences in disordered eating behaviors between different types and sizes of high schools. Once again, various sports could also be compared within these two groups to assess for different scores on the three types of perfectionism and different amounts of disordered eating between the different types of sports.

Measures. Both measures used in the current study have very good reliability and validity and have been used in numerous studies with college populations. The Questionnaire for Eating Disorder Diagnosis (Q-EDD) is also one of the only self-report measures that categorizes participants based on a continuum model of disordered eating. Therefore, limitations surrounding the measures are minimal. The main limitation of the measures is that self-reported height and weight were used in the current study. Future research studies may want to obtain height and weight from a standardized scale measuring these two dimensions. Even though this would be more time consuming than obtaining self-reports of height and weight, it would not only decrease the dishonesty that may occur with self-reporting of height and weight, but would also ensure consistency in the measurements if the same standardized scale was used for every participant.

Future research may also attempt to administer the questionnaires on-line versus inperson. Although this would eliminate the ability to get standardized and consistent height and weight from participants as just discussed, it may make people less likely to give socially desirable response sets if there are not teammates or classmates sitting in the same room as they are completing the questionnaires. Administering the questionnaires on-line may also make it 
more convenient to obtain samples from several colleges and universities across the United States in order to perform comparisons between these different types of colleges and universities in geographically different areas.

Clinical Applications. Although many clinicians are knowledgeable about diagnosable eating disorders, many clinicians are not aware of the distinction between diagnosable eating disorders and disordered eating behaviors. Many times, practitioners will administer an assessment for a diagnosable eating disorder and if a client does not meet the DSM-IV-TR criteria, they will dismiss any relevant behaviors and not focus treatment on these issues. It is imperative that practitioners become more knowledgeable and aware of disordered eating behaviors, the frequency of their occurrence (especially among college student populations), and the possible physical, psychological, and emotional consequences of these behaviors. The results of this study indicate that on average, individuals categorized as symptomatic based on the QEDD have higher levels of both total and maladaptive perfectionism than individuals categorized as asymptomatic. If clinicians were more aware of specific disordered eating behaviors, such as dieting, excessive exercise, and laxative use, among others, they may be more likely to focus treatment not only these behaviors, but also other behaviors, such as perfectionism, that have been found to accompany disordered eating.

Related to this, if a client were to present with issues surrounding perfectionism, specifically maladaptive perfectionism, it is essential that the clinician assess for any possible disordered eating behaviors occurring with the client. Because this study found a statistically significant relationship between scores on perfectionism (total and maladaptive) and disordered eating behaviors, there is a high likelihood that someone who has perfectionistic tendencies will also be engaging in disordered eating behaviors. If a clinician were to not assess for these 
behaviors, they may not treat a key issue of the client. It is possible that individuals who are engaging in disordered eating behaviors could develop a diagnosable eating disorder if the disordered eating behaviors are not treated. If a clinician were to be aware of the disordered eating behaviors and treat them, it is possible that it will help prevent the development of a diagnosable eating disorder in this individual.

Understanding the multidimensional perspective of perfectionism is crucial for clinicians. Becoming aware that there is a negative (maladaptive) type of perfectionism along with a positive (adaptive) type of perfectionism is important in understanding specific client issues that may be associated with perfectionism. For example, if a clinician encounters a college student who is struggling to adjust to college course expectations, they could encourage them to engage in adaptive perfectionistic thoughts and behaviors such as setting realistic goals and then focusing on attaining that goal, rather than maladaptive perfectionistic thoughts and behaviors such as feeling as if they are a complete failure as a person if they receive a low grade on one assignment.

Maladaptive perfectionism may also be associated with several different psychological disorders such as Obsessive-Compulsive Disorder (OCD), Obsessive-Compulsive Personality Disorder, or Major Depressive Disorder among others. Part of treatment plans to address depressive symptoms for example could be making clients aware of the maladaptive perfectionistic thoughts and behaviors they are engaging in, such as believing people will think less of them if they make a mistake. Once clients are aware of these maladaptive perfectionistic thoughts and behaviors, the clinician can work collaboratively with them to change these thoughts and hopefully lessen the depressive symptoms. 
Another clinical application is related to the level of total and maladaptive perfectionism that could possibly be more prevalent in specific populations, including the sample of this study. One of the possible explanations given by the current researcher for not finding a difference in levels of maladaptive and total perfectionism between either males and females or athletes and non-athletes is because of the high standards set at this specific university, as is evidenced by the average high school GPA of incoming freshmen being 3.76 and the average ACT score being 26.58. Although no other colleges or universities were studied, and therefore it is not known that the levels of total and maladaptive perfectionism would be different at different colleges and universities, it is a possibility. If future research was conducted using participants from colleges and universities with differing average incoming GPAs and ACT scores, and it was found that their levels of total and maladaptive perfectionism were lower than that of the current sample, it could have clinical implications. If this were found, practitioners at this university and similar universities would need to be made aware of how maladaptive and total perfectionism can impact other behaviors such as disordered eating. It would also be crucial for practitioners working with these students to assess levels of perfectionism as part of their intake procedures and if levels of maladaptive perfectionism were found to be high, to assess for disordered eating behaviors. If high levels of maladaptive perfectionism are considered "normal" for a certain population, practitioners may not think about this being an issue, and therefore may not assess for related behaviors, such as disordered eating, or make perfectionism or disordered eating one of the focuses of their treatment, even though it may be a serious issue.

Looking at more general clinical applications, although some clinicians have an expertise in disordered eating and/or perfectionism, clinicians in general should become more aware of the different types of perfectionism, the different disordered eating behaviors, and the consequences 
of having perfectionistic attitudes or engaging in disordered eating behaviors in order to not only be able to offer services to those who are displaying these attitudes and behaviors, but also to educate those who have close contact with these individuals. Clinicians can offer workshops, seminars, conferences, and other forms of outreach in order to educate others about the different types of perfectionism, the signs of disordered eating, and the consequences of these attitudes and behaviors, and also to inform others about the pressures that some people may succumb to due to their athletic status or attempt to achieve specific academic standards.

Practitioners might provide treatment to individuals who display high scores on maladaptive and total perfectionism and individuals who are engaging in disordered eating behaviors. As is common in therapy, they might have individuals engage in cognitive restructuring, where they modify their thinking to be more realistic and therefore help individuals to set more realistic goals and to realize when their efforts have been successful, thereby engaging in adaptive perfectionism compared to maladaptive perfectionism, as discussed above. Clinicians might also continue to work with individuals on engaging in cognitive restructuring with relation to their eating behaviors, which may lead to individuals engaging in healthy eating behaviors compared to unhealthy or disordered eating behaviors. Psychoeducation could also continue to be offered to individuals about the negative effects of disordered eating behaviors such as physical consequences like heart arrhythmias, esophageal tears, and organ failure. Practitioners can also continue to provide psychoeducation and training to those in contact with individuals who may be at-risk for perfectionistic attitudes and disordered eating behaviors. For example, practitioners on college campuses could continue to provide training to faculty members, coaches, resident hall staff, athletic trainers, and other college staff on signs to look for when they suspect a student may be engaging in disordered eating behaviors. They can 
also inform these individuals of available resources if they feel that they are in contact with a student who needs professional help. Specifically, practitioners might work with coaches and other athletic personnel on ways to encourage athletes to achieve success in their sport without engaging in disordered eating behaviors or having maladaptive perfectionistic attitudes. Part of this training may include working with coaches and athletic personnel on how to set realistic goals for their athletes, therefore modeling setting realistic expectations to the athletes and hopefully influencing them to set realistic rather than unrealistic goals. Coaches and athletic staff could also be trained on healthy ways for their athletes to obtain and maintain the needed body style for their specific sport, which the coaches and athletic staff could then implement with their athletes, hopefully reducing the risk of disordered eating behaviors. Although these practices may be occurring on large campuses, more of this could take place on smaller campuses.

Finally, some of the same programming and training that is offered to the faculty members, coaches, resident hall staff, and other collegiate staff could also be offered to the students and student-athletes. For example, clinicians could offer a workshop on "Setting Realistic Goals" or "Healthy Body Image" to the students in order to educate individuals on these topics. By giving the individuals the skills and knowledge needed to set realistic goals or maintain a healthy body image, this may decrease the chances of individuals engaging in behaviors associated with maladaptive perfectionistic attitudes or disordered eating behaviors. Again, these programs may be occuring on larger campuses currently, but more of this could be implemented on smaller campuses also.

\section{Summary and Conclusion}

The purpose of the study was to determine the amounts of disordered eating (asymptomatic, symptomatic, and eating disordered) and perfectionism (total, adaptive, and 
maladaptive) in college students. Specifically, the study was designed to establish if scores on the three types of perfectionism varied across the disordered eating categories; if males and females, athletes and non-athletes, and male athletes, female athletes, male non-athletes, and female non-athletes differed in their frequencies in the disordered eating categories (asymptomatic, symptomatic); and if there were significant differences on perfectionism scores (total, maladaptive, adaptive) between males and females, athletes and non-athletes, and the asymptomatic and symptomatic categories of disordered eating.

The findings of the current study were consistent with some of the findings in previous literature and not consistent with other findings in previous literature. Consistent with previous literature, the current study found that scores on total and maladaptive perfectionism varied across disordered eating categories, while scores on adaptive perfectionism did not vary cross disordered eating categories. More specifically, this occurred in a linear fashion with the asymptomatic category having the lowest total and maladaptive perfectionism mean scores and the eating disordered category having the highest total and maladaptive perfectionism mean scores. Also consistent with previous literature, the current findings revealed that males and females significantly differed in their frequencies in the disordered eating categories, with females having a higher frequency than expected in the symptomatic category and males having a higher frequency than expected in the asymptomatic category. Unfortunately, the current study did not find that athletes and non-athletes or that male athletes, female athletes, male nonathletes, and female non-athletes differed in their frequencies in the disordered eating categories. This is similar to some previous research, but in contradiction to most previous research that has found both male and female athletes to have higher frequencies of disordered eating behaviors than male and female non-athletes, respectively, and female athletes to have higher frequencies 
of disordered eating than male athletes. The current study did find that total and maladaptive perfectionism scores differed between the asymptomatic and symptomatic categories of disordered eating, while adaptive perfectionism did not vary between the asymptomatic and symptomatic disordered eating categories. This finding is a new discovery, as this is the first study to use both a continuum model of disordered eating and a multidimensional model of perfectionism. However, contradictory to previous research findings, scores on maladaptive and total perfectionism did not differ between males and females or athletes and non-athletes. The results of this research study lend an enormous level of support for studying disordered eating behaviors on a continuum and perfectionism from a multidimensional perspective.

This is the first study known that used both a continuum model of disordered eating and a multidimensional model of perfectionism when studying these two variables and found that total and maladaptive perfectionism vary across the disordered eating categories, which lends support to using these models/perspectives in future research. To this end, it is imperative that further research be conducted using this model and perspective in order to increase knowledge and understanding of these two variables. Several recommendations and suggestions were given for future research.

Disordered eating behaviors and maladaptive perfectionistic attitudes are prevalent on both college campuses and in our society in general. Clinicians are encouraged to increase their knowledge about the different types of perfectionism and the different disordered eating behaviors in order to educate others about these topics. Clinicians are also urged to offer services to individuals who may be having difficulty with these issues and also to offer training and education to those that work closely with individuals who may be susceptible to maladaptive perfectionistic thinking and engagement in disordered eating behaviors. 


\section{References}

American Psychiatric Association. (2000). Diagnostic and statistical manual of mental disorders:

DSM-IV-TR. Washington, DC: American Psychiatric Association.

American Psychological Association. (2010). Publication manual of the American Psychological Association (6 ${ }^{\text {th }}$ ed.). Washington D. C.: Author

Ashby, J. S., Kottman, T., \& Schoen, E. (1998). Perfectionism and eating disorders reconsidered. Journal of Mental Health Counseling, 20(3), 261-271.

Bardone-Cone, A. M., Wonderlich, S. A., Frost, R. O., Bulik, C. M., Mitchell, J. E., Uppala, S., \& Simonich, H. (2007). Perfectionism and eating disorders: Current status and future directions. Clinical Psychology Review, 27(3), 384-405.

Bastiani, A. M., Rao, R., Weltzin, T., \& Kaye, W. H. (1995). Perfectionism in anorexia nervosa. International Journal of Eating Disorders, 17(2), 147-152.

Blouin, A. G. \& Goldfield, G. S. (1995). Body image and steroid use in male bodybuilders. International Journal of Eating Disorders, 18(2), 159-165.

Bulik, C. M., Tozzi, F., Anderson, C., Mazzeo, S. E., Aggen, S., \& Sullivan, P. F. (2003). The relation between eating disorders and components of perfectionism. American Journal of Psychiatry, 160(2), 366-368.

Byrne, S. \& McLean, N. (2001). Eating disorders in athletes: A review of the literature. Journal of Science and Medicine in Sport, 4(2), 145-159.

Clavin, S. L., Clavin, R. H., Gayton, W. F., \& Broida, J. (1996). Continued validation of the multidimensional perfectionism scale. Psychological Reports, 78(3, Pt 1), 732-734.

Cohen, D. L. \& Petrie, T. A. (2005). An examination of psychosocial correlates of disordered eating among undergraduate women. Sex Roles, 52(1/2), 29-42. 
Cozby, P. C., \& Bates, S. C. (2012). Methods in behavioral research (11 $1^{\text {th }}$ Ed.). New York, NY: McGraw-Hill Companies.

Crisp, A. H. \& Burns, T. (1990). Primary anorexia nervosa in the male and female: A comparison of clinical features and prognosis. In A. E. Andersen (Ed.), Males with eating disorders (pp. 77-99). Philadelphia, PA: Brunner/Mazel, Inc.

Davis, C., Claridge, G., \& Fox, J. (2000). Not just a pretty face: Physical attractiveness and perfectionism in the risk for eating disorders. International Journal of Eating Disorders, $27(1), 67-73$.

Edwin, D. H. \& Andersen, A. E. (1990). Psychometric testing in 76 males with eating disorders. In A. E. Andersen (Ed.), Males with eating disorders (pp. 116-130). Philadelphia, PA: Brunner/Mazel, Inc.

Field, A. E., Cheung, L., Wolf, A. M., Herzog, D. B., Gortmaker, S. L., \& Colditz, G. A. (1999). Exposure to the mass media and weight concerns among girls. Pediatrics, 103(3), $1-2$.

Forbush, K., Heatherton, T. F., \& Keel, P. K. (2007). Relationships between perfectionism and specific disordered eating behaviors. International Journal of Eating Disorders, 40(1), 37-41.

Franco-Paredes, K., Mancilla-Diaz, J. M., Vazquez-Arevalo, R., Lopez-Aguilar, X., \& Alvarez-Rayon, G. (2005). Perfectionism and eating disorders: A review of the literature. European Eating Disorders Review, 13(1), 61-70.

Frost, R. O., Heimberg, R. G., Holt, C. S., Mattia, J. I., et al. (1993). A comparison of two measures of perfectionism. Personality \& Individual Differences, 14(1), 119-126.

Frost, R. O., Marten, P., Lahart, C., \& Rosenblate, R. (1990). The dimensions of 
perfectionism. Cognitive Therapy and Research, 14(5), 449-468.

Fulkerson, J. A., Keel, P. K., Leon, G. R., \& Dorr, T. (1999). Eating-disordered behaviors and personality characteristics of high school athletes and nonathletes. International Journal of Eating Disorders, 26(1), 73-79.

Furnham, A., Badmin, N., \& Sneade, I. (2002). Body image dissatisfaction: Gender differences in eating attitudes, self-esteem, and reasons for exercise. Journal of Psychology: Interdisciplinary and Applied, 136(6), 581-596.

Gary, A. (2001). Pathophysiology of eating disorders. In J. J. Robert-McComb (Ed.), Eating disorders in women and children: Prevention, stress management, and treatment (pp. 49-58). Boca Raton, FL: CRC Press.

Gravetter, F. J., \& Wallnau, L. B. (1996). Statistics for the behavioral sciences (4 ${ }^{\text {th }}$ Ed.). St. Paul, MN: West Publishing Company.

Grigg, M., Bowman, J., \& Redman, S. (1996). Disordered eating and unhealthy weight reduction practices among adolescent females. Preventative Medicine: An International Journal Devoted to Practice \& Theory, 25(6), 748-756.

Halmi, K. A., Sunday, S. R., Strober, M., Kaplan, A., Woodside, D. B., Fichter, M.,...Kaye, W. H. (2000). Perfectionism in anorexia nervosa: Variation by clinical subtype, obsessionality, and pathological eating behavior. American Journal of Psychiatry, 157(11), 1799-1805.

Hamachek, D. E. (1978). Psychodynamics of normal and neurotic perfectionism. Psychology: A Journal of Human Behavior, 15(1), 27-33.

Hausenblas, H. A., \& Carron, A. V. (1999). Eating disorder indices and athletes: An integration. Journal of Sport \& Exercise Psychology, 21, 230-258. 
Hay, P. J., Loukas, A., \& Philpott, H. (2005). Prevalence and characteristics of men with eating disorders in primary care: How do they compare to women and what features may aid in identification? Primary Care \& Community Psychiatry, 10(1), 1-6.

Hewitt, P. L., Flett, G. L., \& Ediger, E. (1995). Perfectionism traits and perfectionistic selfpresentation in eating disorder attitudes, characteristics, and symptoms. International Journal of Eating Disorders, 18(4), 317-326.

Howell, D. C. (2002). Statistical methods for psychology ( $5^{\text {th }}$ Ed.). Pacific Grove, CA: Duxbury.

Johnson, C., Powers, P. S., \& Dick, R. (1999). Athletes and eating disorders: The national collegiate athletic association study. International Journal of Eating Disorders, 26, 179188.

Joiner, T. E., Heatherton, T. F., Rudd, M. D., \& Schmidt, N. B. (1997). Perfectionism, perceived weight status, and bulimic symptoms: Two studies testing a diathesis-stress model. Journal of Abnormal Psychology, 106(1), 145-153.

Joiner, T. E. Jr., Katz, J., \& Heatherton, T. F. (2000). Personality features differentiate late adolescent females and males with chronic bulimic symptoms. International Journal of Eating Disorders, 27(2), 191-197.

Kashubeck-West, S., Mintz, L. B., \& Saunders, K. J. (2001). Assessment of eating disorders in women. Counseling Psychologist, 29(5), 662-694.

Kashubeck-West, S. \& Saunders, K. (2001). Inventories used to assess eating disorder symptomatology in clinical and nonclinical settings. In J. J. Robert-McComb (Ed.), Eating disorders in women and children: Prevention, stress management, and treatment (pp. 59-85). Boca Raton, FL: CRC Press.

Kearney-Cooke, A. \& Steichen-Asch, P. (1990). Men, body image, and eating disorders. In 
A. E. Andersen (Ed.), Males with eating disorders (pp. 54-74). Philadelphia, PA: Brunner/Mazel, Inc.

Macedo, A., Soares, M. J., Azevedo, M. H., Gomes, A., Pereira, A. T., Maia, B., \& Pato, M. (2007). Perfectionism and eating attitudes in Portuguese university students. European Eating Disorders Review, 15(4), 296-304.

McCabe, M. P. \& Vincent, M. A. (2003). The role of biodevelopmental and psychological factors in disordered eating among adolescent males and females. European Eating Disorders Review, 11(4), 315-328.

McVey, G. L., Pepler, D., Davis, R., Flett, G. L., \& Abdolell, M. (2002). Risk and protective factors associated with disordered eating during early adolescence. Journal of Early Adolescence, 22(1), 75-95.

Mickalide, A. D. (1990). Sociocultural factors influencing weight among males. In A. E. Andersen (Ed.), Males with eating disorders (pp. 30-39). Philadelphia, PA: Brunner/Mazel, Inc.

Mintz, L. B. \& Betz, N. E. (1988). Prevalence and correlates of eating disordered behaviors among undergraduate women. Journal of Counseling Psychology, 35(4), 463-471.

Mintz, L. B., O’Halloran, M. S., Mulholland, A. M., \& Schneider, P. A. (1997).

Questionnaire for eating disorder diagnoses: Reliability and validity of operationalizing DSM-IV criteria into a self-report format. Journal of Counseling Psychology, 44(1), 63-79.

O’Dea, J. A. \& Abraham, S. (2002). Eating and exercise disorders in young college men. Journal of American College Health, 50(6), 273-278.

Parker, W. D. \& Adkins, K. K. (1995). A psychometric examination of the 
multidimensional perfectionism scale. Journal of Psychopathology and Behavioral Assessment, 17(4), 323-334.

Petrie, T. A. \& Rogers, R. (2001). Extending the discussion of eating disorders to include men and athletes. The Counseling Psychologist, 29(5), 743-753.

Pliner, P. \& Haddock, G. (1996). Perfectionism in weight-concerned and unconcerned women: An experimental approach. International Journal of Eating Disorders, 19(4), 381-389.

Pratt, E. M., Telch, C. F., Labouvie, E. W., Wilson, G. T., \& Agras, W. S. (2001). Perfectionism in women with binge eating disorder. International Journal of Eating Disorders, 29(2), 177-186.

Presnell, K. Bearman, S. K., \& Madeley, M. C. (2007). Body dissatisfaction in adolescent females and males: Risk and resilience. Prevention Researcher, 14(3), 3-6.

Pritchard, M. E., Milligan, B., Elgin, J., Rush, P., \& Shea, M. (2007). Comparisons of risky health behaviors between male and female college athletes and non-athletes. Athletic Insight: The Online Journal of Sport Psychology, 9(1), 1-11.

Robb, A. S. \& Dadson, M. J. (2002). Eating disorders in males. Child \& Adolescent Psychiatric Clinics of North America, 11(2), 399-418.

Rohwer, J. \& Massey-Stokes, M. S. (2001). Factors associated with eating disorders in children. In J. J. Robert-McComb (Ed.), Eating disorders in women and children: Prevention, stress management, and treatment (pp. 211-224). Boca Raton, FL: CRC Press.

Rosenvinge, J. H., Borgen, J. S., \& Borresen, R. (1999). The prevalence and psychological 
correlates of anorexia nervosa, bulimia nervosa, and binge eating among 15-year-old students: A controlled epidemiological study. European Eating Disorders Review, 7(5), 382-391.

Slane, J. D., Burt, S. A., \& Klump, K. L. (2010). The road less traveled: Associations between externalizing behaviors and eating pathology. International Journal of Eating Disorders, 43(2), 149-160.

Smolak, L., Murnen, S. K., \& Ruble, A. E. (2000). Female athletes and eating problems: A meta-analysis. International Journal of Eating Disorders, 27(4), 371-379.

Sundgot-Borgen, J. \& Torstveit, M. K. (2004). Prevalence of eating disorders in elite athletes is higher than in the general population. Clinical Journal of Sport Medicine, 14(1), 25-35.

Szymanski, L. A. \& Chrisler, J. C. (1990-1991). Eating disorders, gender-role and athletic activity. Psychology: A Journal of Human Behavior, 27(4)-28(1), 20-29.

Tabachnick, B. G., \& Fidell, L. S. (2001). Using multivariate statistics (4 $4^{\text {th }}$ Ed.). Needham Heights, MA: Allyn \& Bacon.

Tylka, T. L. \& Subich, L. M. (1999). Exploring the construct validity of the eating disorder continuum. Journal of Counseling Psychology, 46(2), 268-276.

Tylka, T. L. \& Subich, L. M. (2002). A preliminary investigation of the eating disorder continuum with men. Journal of Counseling Psychology, 49(2), 273-279.

Varnado-Sullivan, P. J., Horton, R., \& Savoy, S. (2006). Differences for gender, weight, and exercise in body image disturbance and eating disorder symptoms. Eating and Weight Disorders, 11(3), 118-125.

Vohs, K. D., Bardone, A. M., Joiner, T. E. Jr., \& Abramson, L. Y. (1999). Perfectionism, perceived weight status, and self-esteem interact to predict bulimic symptoms: A model 
of bulimic symptom development. Journal of Abnormal Psychology, 108(4), 695-700.

Woodside, D. B., Bulik, C. M., Thornton, L., Klump, K. L., Tozzi, F., Fichter, M. M.,... \& Kaye, W. H. (2004). Personality in men with eating disorders. Journal of Psychosomatic Research, 57(3), 273-278.

Woodside, D. B., Garner, D. M., Rockert, W., \& Garfinkel, P. E. (1990). Eating disorders in males: Insights from a clinical and psychometric comparison with female patients. In A. E. Andersen (Ed.), Males with eating disorders (pp. 100-115). Philadelphia, PA: Brunner/Mazel, Inc. 
Appendices 
Appendix A

Questionnaires 


\section{Questionnaire for Eating Disorder Diagnosis (Q-EDD)}

Please complete the following questions as honestly as possible. The questions refer to current behaviors and beliefs, meaning those that have occurred in the past 3 months.

Sex: $\quad$ (Please circle) $\quad$ Male $\quad$ Female

Age:

School/Occupational Status: $\quad$ (Please circle)

Junior High or younger (specify grade:

High School Freshman

High School Sophomore

High School Junior

High School Senior

College Freshman

College Sophomore

College Junior

College Senior

Not in School/Employed (specify:

Race/Ethnicity: Caucasian/White

(Please circle) African-American/Black

Hispanic/Latino/Mexican-American

American Indian

Asian American/Pacific Islander

Other : (specify)

Present height: feet inches

Present weight: pounds

My body-frame is: small medium large (Please circle)

I would like to weigh pounds 
1. Do you experience recurrent episodes of binge eating, meaning eating in a discrete period of time (e.g., within any 2-hour period) an amount of food that is definitely larger than most people would eat during a similar time period?

YES NO
If YES:
Continue to answer the following questions

If NO:

Skip to Question \#4 (on the next page)

2. Do you have a sense of lack of control during the binge eating episodes (i.e., the feeling that you cannot stop eating or control what or how much you are eating)?

YES NO

3. Circle the answers within the two sets of [bold brackets] below that best fit for you:

On the average, I have had $[1,2,3,4,5,6$ or more $]$ binge eating episodes a WEEK for at least [1 month, 2 months, 3 months, 4 months, 5 months, 6-12 months, more than one year]

4. Please circle the appropriate responses below concerning things you may do currently to prevent weight gain. If you circle yes to any question, please indicate how often on the average you do this and how long you have been doing this.

a) Do you make yourself vomit to prevent weight gain? YES NO How often do you do this?

Daily Twice/Week Once/Week Once/Month

How long have you been doing this?

1 month 2 months $\quad 3$ months 4 months $5-11$ months More than a year

b) Do you take laxatives to prevent weight gain? YES NO

How often do you do this?

Daily Twice/Week Once/Week Once/Month

How long have you been doing this?

1 month 2 months $\quad 3$ months 4 months $5-11$ months More than a year

c) Do you take diuretics (water pills) to prevent weight gain? YES NO

How often do you do this?

Daily Twice/Week Once/Week Once/Month

How long have you been doing this?

1 month 2 months 3 months 4 months 5-11 months More than a year

d) Do you fast (skip food for 24 hours) to prevent weight gain? YES NO

How often do you do this?

Daily Twice/Week Once/Week Once/Month

How long have you been doing this?

1 month 2 months $\quad 3$ months 4 months 5-11 months More than a year 
e) Do you chew food but spit it out to prevent weight gain? YES NO How often do you do this?

Daily Twice/Week Once/Week Once/Month

How long have you been doing this?

1 month 2 months 3 months 4 months 5-11 months More than a year

f) Do you give yourself an enema to prevent weight gain? YES NO

How often do you do this?

Daily Twice/Week Once/Week Once/Month

How long have you been doing this?

1 month 2 months $\quad 3$ months 4 months $5-11$ months More than a year

g) Do you take appetite control pills to prevent weight gain? YES NO

How often do you do this?

Daily Twice/Week Once/Week Once/Month

How long have you been doing this?

1 month 2 months 3 months 4 months 5-11 months More than a year

h) Do you diet strictly to prevent weight gain? YES NO

How often do you do this?

Daily Twice/Week Once/Week Once/Month

How long have you been doing this?

1 month 2 months $\quad 3$ months 4 months 5-11 months More than a year

i) Do you exercise a lot? YES NO

How often do you do this?

Daily Twice/Week Once/Week Once/Month

How long have you been doing this?

1 month 2 months $\quad 3$ months $\quad 4$ months 5-11 months More than a year

5. If you answered YES to "exercise a lot" please answer questions \#5a, 5b, 5c, and 5d. If you answered NO to "exercise a lot," skip to question \#6.

5a. Fill in the blanks below:

I (types of exercise,

e.g., jog, swim) for an average of hours at a time.

5b. My exercise sometimes significantly interferes with important activities. YES NO

5c. I exercise despite injury and/or medical complications. YES NO

5d. Is your primary reason for exercising to counteract the effects of binges or to prevent weight gain? YES NO 
For the following questions, circle the response that best reflects your answer:

6. Does your weight and/or body shape influence how you feel about yourself?

1

Not at all A Little
3

A moderate amoun
4

Very Much
5

Extremely or Completely

7. How afraid are you of becoming fat?

1

Not at all A Little
3

A moderate amoun
4

Very Much
5

Extremely or Completely

8. How afraid are you of gaining weight?

1

Not at all A Little
3

A moderate amoun
4

Very Much
5

Extremely or Completely

9. Do you consider yourself to be:

$\begin{array}{llllll}1 & 2 & 3 & 4 & 5 & 6 \\ \begin{array}{l}\text { Grossly } \\ \text { Obese }\end{array} & \begin{array}{l}\text { Moderately } \\ \text { Obese }\end{array} & \text { Overweight } & \text { Normal } & \text { Low } & \text { Severely } \\ & & \text { Weight } & \text { Weight } & \text { Underweight }\end{array}$

10. Certain parts of my body (e.g., my abdomen, buttocks, thighs) are too fat. $\quad$ YES NO

11. I feel fat all over. YES NO

12. I believe that how little I weigh is a serious problem. YES NO

13. I have missed at least 3 consecutive menstrual cycles (not including those missed during a pregnancy). YES NO 


\section{Frost Multidimensional Perfectionism Scale (Frost MPS)}

Please circle the response that best describes you.

1 = Strongly Disagree (SD), 2 = Disagree (D), 3 = Neutral (N), 4 = Agree (A), 5 = Strongly Agree (SA)

1. My parents set very high standards for me

$\begin{array}{lllll}1 & 2 & 3 & 4 & 5\end{array}$

2. Organization is very important to me.

$\begin{array}{lllll}1 & 2 & 3 & 4 & 5\end{array}$

3. As a child, I was punished for doing things less

$\begin{array}{lllll}1 & 2 & 3 & 4 & 5\end{array}$
than perfect.

4. If I do not set the highest standards for myself, I am likely to end up a second-rate person.

5. My parents never tried to understand my mistakes. $\quad \begin{array}{lllll}1 & 2 & 3 & 4 & 5\end{array}$

6. It is important to me that I be thoroughly competent in everything I do.

7. I am a neat person.

$\begin{array}{lllll}1 & 2 & 3 & 4 & 5\end{array}$

8. I try to be an organized person.

$\begin{array}{lllll}1 & 2 & 3 & 4 & 5\end{array}$

9. If I fail at work/school, I am a failure as a person.

$\begin{array}{lllll}1 & 2 & 3 & 4 & 5\end{array}$

10. I should be upset if I make a mistake.

$\begin{array}{lllll}1 & 2 & 3 & 4 & 5\end{array}$

11. My parents wanted me to be the best at everything. $\quad \begin{array}{llllll}1 & 2 & 3 & 4 & 5\end{array}$

12. I set higher goals than most people.

$\begin{array}{lllll}1 & 2 & 3 & 4 & 5\end{array}$


1 = Strongly Disagree (SD), 2 = Disagree (D), 3 = Neutral (N), 4 = Agree (A), 5 = Strongly Agree (SA)

13. If someone does a task at work/school better than $\quad \begin{array}{llllll}1 & 2 & 3 & 4 & 5\end{array}$ I, then I feel like I failed the whole task.

14. If I fail partly, it is as bad as being a complete failure. $\quad \begin{array}{lllll}1 & 2 & 3 & 4 & 5\end{array}$

15. Only outstanding performance is good enough $\quad \begin{array}{llllll}1 & 2 & 3 & 4 & 5\end{array}$ in my family.

16. I am very good at focusing my efforts on $\begin{array}{lllll}1 & 2 & 3 & 4 & 5\end{array}$ attaining a goal.

17. Even when I do something very carefully, I $\quad \begin{array}{llllll}1 & 2 & 3 & 4 & 5\end{array}$ often feel that it is not quite right.

18. I hate being less than the best at things. $\quad \begin{array}{llllll}1 & 2 & 3 & 4 & 5\end{array}$

19. I have extremely high goals. $\quad \begin{array}{llllll} & 1 & 2 & 3 & 4 & 5\end{array}$

20. My parents have expected excellence from me. $\quad \begin{array}{llllll}1 & 2 & 3 & 4 & 5\end{array}$

21. People will probably think less of me if $\quad \begin{array}{llllll}1 & 2 & 3 & 4 & 5\end{array}$ I make a mistake.

22. I never felt like I could meet my parents' expectations. $1 \quad \begin{array}{llll}2 & 3 & 4 & 5\end{array}$

23. If I do not do as well as other people, it means I am $\quad \begin{array}{llllll}1 & 2 & 3 & 4 & 5\end{array}$ an inferior human being.

24. Other people seem to accept lower standards $\quad \begin{array}{llllll}1 & 2 & 3 & 4 & 5\end{array}$ from themselves than I do. 
1 = Strongly Disagree (SD), 2 = Disagree (D), 3 = Neutral (N), 4 = Agree (A), 5 = Strongly Agree (SA)

25. If I do not do well all the time, people will not respect me.

26. My parents have always had higher expectations for my future than I have.

27. I try to be a neat person.

$\begin{array}{lllll}1 & 2 & 3 & 4 & 5\end{array}$

28. I usually have doubts about the simple everyday

$\begin{array}{lllll}1 & 2 & 3 & 4 & 5\end{array}$
things I do.

29. Neatness is very important to me.

$\begin{array}{lllll}1 & 2 & 3 & 4 & 5\end{array}$

30. I expect higher performance in my daily tasks than most people.

$\begin{array}{lllll}1 & 2 & 3 & 4 & 5\end{array}$

31. I am an organized person.

$\begin{array}{lllll}1 & 2 & 3 & 4 & 5\end{array}$

32. I tend to get behind in my work because I repeat

$\begin{array}{lllll}1 & 2 & 3 & 4 & 5\end{array}$
things over and over.

33. It takes me a long time to do something "right." $\quad \begin{array}{llllll}1 & 2 & 3 & 4 & 5\end{array}$

34. The fewer mistakes I make, the more people will $\quad \begin{array}{llllll}1 & 2 & 3 & 4 \quad 5\end{array}$ like me.

35. I never felt like I could meet my parents' standards. $\quad \begin{array}{llllll}1 & 2 & 3 & 4 & 5\end{array}$ 
Appendix B

Data Analyses Graphs 
Research Question 1a: Do scores on total perfectionism vary across disordered eating categories (asymptomatic, symptomatic, eating disordered)?

\section{ANOVA}

Disordered eating category $-\mathrm{A}=$ =Asymptomatic, $\mathrm{S}=$ Symptomatic, $\mathrm{ED}=$ Eating Disordered Perfectionism scores $-\mathrm{T}=$ Total

\begin{tabular}{|l|l|l|l|}
\hline & $\mathbf{A}$ & $\mathbf{S}$ & $\mathbf{E D}$ \\
\hline $\mathbf{T}$ & & & \\
\hline
\end{tabular}

Research Question 1b: Do scores on maladaptive perfectionism vary across disordered eating categories (asymptomatic, symptomatic, eating disordered)?

\section{ANOVA}

Disordered eating category $-\mathrm{A}=$ =Asymptomatic, $S=$ =Symptomatic, ED=Eating Disordered Perfectionism scores $-\mathrm{M}=$ Maldaptive

\begin{tabular}{|l|l|l|l|}
\hline & $\mathbf{A}$ & $\mathbf{S}$ & $\mathbf{E D}$ \\
\hline $\mathbf{M}$ & & & \\
\hline
\end{tabular}

Research Question 1c: Do scores on adaptive perfectionism vary across disordered eating categories (asymptomatic, symptomatic, eating disordered)?

\section{ANOVA}

Disordered eating category $-\mathrm{A}=$ =Asymptomatic, $\mathrm{S}=$ =Symptomatic, ED=Eating Disordered Perfectionism scores $-\mathbf{A}=$ Adaptive

\begin{tabular}{|l|l|l|l|}
\hline & $\mathbf{A}$ & $\mathbf{S}$ & $\mathbf{E D}$ \\
\hline $\mathbf{A}$ & & & \\
\hline
\end{tabular}


Research Question 2a: Does a difference exist in the frequencies between males' and females' in the disordered eating categories (asymptomatic, symptomatic)?

\section{Chi-square}

Disordered eating categories $-\mathrm{A}=\mathrm{Asymptomatic}, \mathrm{S}=$ symptomatic

Sex - M=Males, $F=$ Females

\begin{tabular}{|l|l|l|}
\hline & $\mathbf{A}$ & $\mathbf{S}$ \\
\hline $\mathbf{M}$ & & \\
\hline $\mathbf{F}$ & & \\
\hline
\end{tabular}

Research Question 2b: Does a difference exist in the frequencies between non-athletes' and athletes' in the disordered eating categories (asymptomatic, symptomatic)?

Chi-square of independence

Disordered eating categories $-\mathrm{A}=$ Asymptomatic, $\mathrm{S}=$ =symptomatic

Athletic Status $-A=A t h l e t e s, ~ N A=N o n-A t h l e t e s$

\begin{tabular}{|l|l|l|}
\hline & $\mathbf{A}$ & $\mathbf{S}$ \\
\hline $\mathbf{A}$ & & \\
\hline NA & & \\
\hline
\end{tabular}


Research Question 2c: Does a difference exist in the frequencies between male athletes', female athletes', male non-athletes', and female non-athletes' in the disordered eating categories (asymptomatic, symptomatic)?

\section{Chi-square of independence}

Disordered eating categories $-\mathrm{A}=$ Asymptomatic, $\mathrm{S}=$ =symptomatic

Male Athletes=MA, Female Athletes=FA, Male Non-Athletes=MNA, Female NonAthletes=FNA

\begin{tabular}{|c|c|c|}
\hline & A & S \\
\hline MA & & \\
\hline FA & & \\
\hline MNA & & \\
\hline FNA & & \\
\hline
\end{tabular}


Research Question 3a: Are there any significant interactions on total perfectionism scores between or among the independent variables of sex, athletic status, and disordered eating category?

Research Question 4a: Is there a significant difference on total perfectionism scores between males and females?

Research Question 5a: Is there a significant difference on total perfectionism scores between athletes and non-athletes?

Research Question 6a: Is there a significant difference on total perfectionism scores between the asymptomatic and symptomatic categories of disordered eating?

\section{One $2 \times 2 \times 2$ ANOVA}

Perfectionism T=Total; Sex (M=Male, F=Female); Athletic Status(AS) (A=Athlete, NA=Non-Athlete); Disordered Eating Category(DE) (A=Asymptomatic, S=Symptomatic)

\begin{tabular}{|c|c|c|c|}
\hline & & & T Mean \\
\hline Sex & AS & DE & \\
\hline M & A & A & \\
\hline & & S & \\
\hline & & Total & \\
\hline & NA & A & \\
\hline & & S & \\
\hline & & Total & \\
\hline & Total & A & \\
\hline & & S & \\
\hline & & Total & \\
\hline F & A & A & \\
\hline
\end{tabular}




\begin{tabular}{|c|c|c|c|c|}
\hline & & $\bar{S}$ & & \\
\hline & & Total & & \\
\hline & NA & $\bar{A}$ & & \\
\hline & & $\mathbf{S}$ & & \\
\hline & & Total & & \\
\hline & Total & $\bar{A}$ & & \\
\hline & & $S$ & & \\
\hline & & Total & & \\
\hline Total & $\overline{\mathbf{A}}$ & $\bar{A}$ & & \\
\hline & & $\mathbf{S}$ & & \\
\hline & & Total & & \\
\hline & NA & A & & \\
\hline & & $\mathbf{S}$ & & \\
\hline & & Total & & \\
\hline & Total & $\overline{\mathbf{A}}$ & & \\
\hline & & $\mathbf{S}$ & & \\
\hline & & Total & & \\
\hline
\end{tabular}


Research Question 3b: Are there any significant interactions on maladaptive or adaptive perfectionism scores between or among the independent variables of sex, athletic status, and disordered eating category?

Research Question 4b: Is there a significant difference on maladaptive or adaptive perfectionism scores between males and females?

Research Question 5b: Is there a significant difference on maladaptive or adaptive perfectionism scores between athletes and non-athletes?

Research Question 6b: Is there a significant difference on maladaptive or adaptive perfectionism scores between the asymptomatic and symptomatic categories of disordered eating?

\section{One 2x2x2 MANOVA}

Perfectionism (M=Maladaptive, A=Adaptive); Sex (M=Male, F=Female); Athletic

Status(AS) (A=Athlete, NA=Non-Athlete); Disordered Eating Category(DE) (A=Asymptomatic, $\mathrm{S}=$ Symptomatic)

\begin{tabular}{|c|c|c|c|c|}
\hline & & & M Mean & A Mean \\
\hline Sex & AS & DE & & \\
\hline M & A & A & & \\
\hline & & S & & \\
\hline & & Total & & \\
\hline & NA & A & & \\
\hline & & S & & \\
\hline & & Total & & \\
\hline & Total & A & & \\
\hline & & S & & \\
\hline & & Total & & \\
\hline
\end{tabular}




\begin{tabular}{|c|c|c|c|c|}
\hline F & A & A & & \\
\hline & & S & & \\
\hline & & Total & & \\
\hline & NA & A & & \\
\hline & & S & & \\
\hline & Total & A & & \\
\hline & & S & & \\
\hline & & Total & & \\
\hline Total & A & A & & \\
\hline & & S & & \\
\hline & & Total & & \\
\hline & NA & A & & \\
\hline & & S & & \\
\hline & & Total & & \\
\hline & Total & A & & \\
\hline & & S & & \\
\hline & & Total & & \\
\hline
\end{tabular}

